\title{
One-loop renormalized Higgs boson vertices in the Georgi-Machacek model
}

\author{
Cheng-Wei Chiang, ${ }^{1,2,3, *}$ An-Li Kuo, ${ }^{3, \dagger}$ and Kei Yagyu ${ }^{4, \$}$ \\ ${ }^{1}$ Department of Physics and Center for Theoretical Physics, National Taiwan University, \\ Taipei, Taiwan 10617, Republic of China \\ ${ }^{2}$ Institute of Physics, Academia Sinica, Taipei, Taiwan 11529, Republic of China \\ ${ }^{3}$ Department of Physics and Center of High Energy and High Field Physics, National Central University, \\ Chungli, Taiwan 32001, Republic of China \\ ${ }^{4}$ Seikei University, Musashino, Tokyo 180-8633, Japan
}

(Received 17 May 2018; published 27 July 2018)

\begin{abstract}
We compute renormalized vertices of the $125 \mathrm{GeV}$ Higgs boson $h$ with the weak gauge bosons $(h V V)$, fermions $(h f \bar{f})$, and itself $(h h h)$ in the Georgi-Machacek model at one-loop level. The renormalization is performed based on the on-shell scheme with the use of the $\overline{\mathrm{MS}}$ scheme only for the $h h h$ vertex. We explicitly show the gauge dependence in the counterterms of the scalar mixing parameters in the general $R_{\xi}$ gauge and that the dependence can be removed by using the pinch technique in physical scattering processes. We then discuss the possible allowed deviations in these one-loop corrected Higgs couplings from the standard model predictions by scanning model parameters under the constraints of perturbative unitarity and vacuum stability as well as those from experimental data.
\end{abstract}

DOI: 10.1103/PhysRevD.98.013008

\section{INTRODUCTION}

Discovery of the $125 \mathrm{GeV}$ Higgs boson at the CERN Large Hadron Collider (LHC) completed the particle spectrum of the standard model (SM). This, however, does not necessarily mean that the SM is the ultimate theory describing elementary particle physics, because of theoretically unsatisfactory issues, such as the gauge hierarchy problem and unexplained phenomena related to neutrino mass, dark matter, and the baryon asymmetry of the Universe. These problems are expected to be solved in new physics (NP) beyond the SM at or above the TeV scale. In NP models, the Higgs sector is often extended from the minimal form assumed in the SM, and its properties strongly depend on the NP scenario. Therefore, a determination of the structure of the Higgs sector using experimental data is important to narrow down possible NP models.

There are basically two ways to identify an extended Higgs sector: the direct search and the indirect search. The former approach is to discover additional Higgs bosons, while the latter is to find deviations in various observables related to the discovered Higgs boson $(h)$ from the SM

\footnotetext{
*chengwei@phys.ntu.edu.tw

†101222028@cc.ncu.edu.tw

"yagyu@st.seikei.ac.jp
}

Published by the American Physical Society under the terms of the Creative Commons Attribution 4.0 International license. Further distribution of this work must maintain attribution to the author(s) and the published article's title, journal citation, and DOI. Funded by SCOAP ${ }^{3}$. predictions. So far, no additional Higgs boson has been discovered at the LHC, and this situation makes the indirect search attractive. Currently, the Higgs boson couplings are measured with insufficient accuracies at the LHC, e.g., a $10 \%$ level uncertainty in the $h V V\left(V=W^{ \pm}, Z\right)$ couplings [1]. They are expected to be measured with much better accuracies in future collider experiments, such as the highluminosity LHC and $e^{+} e^{-}$colliders, where they can be determined to the percent or subpercent level $[2,3]$.

In order to make a sensible comparison with such precision measurements, one needs to reduce theoretical uncertainties in models with an extended Higgs sector. In particular, radiative corrections to the Higgs boson couplings should be taken into account. One-loop corrections to various Higgs boson couplings have been studied in several models with a simple Higgs extension, e.g., models with an additional isospin singlet scalar field, the Higgs singlet model (HSM) [4-7], a doublet scalar field, twoHiggs doublet models (THDMs) [8-11], and a complex triplet field, the Higgs triplet model (HTM) [12,13]. Recently, a numerical tool H-COUP [14] has been constructed to compute various $h$ couplings at one-loop level in the HSM and THDMs without any gauge dependence [15].

In this paper, we investigate one-loop corrections to the Higgs boson couplings in the Georgi-Machacek (GM) model $[16,17]$ which has the capacity to provide Majorana mass to left-handed neutrinos through the type-II seesaw mechanism as in the HTM [18-20]. This model realizes the minimal Higgs sector containing isospin triplet scalar fields while having an approximate 
custodial symmetry ${ }^{1}$ in the scalar sector. Thanks to this custodial symmetry, the vacuum expectation value (VEV) of the Higgs triplet fields is allowed to be sizeable while keeping the electroweak rho parameter $\rho=1$ at tree level, a desirable property that is consistent with experimental observations [21]. This property can provide phenomenologically interesting predictions. For example, the $h V V$ couplings can be larger than their SM values, leading to enhanced weak gauge boson scattering processes via the SM-like and exotic Higgs bosons [22,23]. It has also been shown that with an $\mathcal{O}(10)-\mathrm{GeV}$ triplet VEV, the model allows a sufficiently strong first-order phase transition to facilitate successful electroweak baryogenesis [24].

In our earlier work [25], the one-loop corrected $h V V$ couplings had been calculated in the GM model without presenting the details. Besides, the Yukawa couplings $(h f \bar{f})$ and the Higgs self-coupling $(h h h)$ were not computed in that work. In this paper, we present in detail our computations of all these $h$ couplings (i.e., $h V V, h f \bar{f}$, and $h h h)$ at one-loop level. We apply the on-shell renormalization scheme to our calculation, where counterterms for scalar mixing parameters remain gauge-dependent as been generally shown for models with mixing among scalar fields $[26,27]$. We discuss how such a gauge dependence can be removed by using the pinch technique for physical processes.

The structure of this paper is as follows. In Sec. II, we briefly review the GM model, separately discussing the scalar potential, the scalar kinetic energies, and the Yukawa interactions. We also discuss the decoupling limit of the model. Section III is devoted to the discussions of renormalization in each of the gauge, fermion, and scalar sectors. We introduce the necessary counterterms and renormalization conditions to determine these counterterms. Section IV takes special care of the gauge dependence issue in some scalar two-point functions. We will adopt the general $R_{\xi}$ gauge in the computations, and employ the pinch technique to remove the gauge dependence in physical scattering processes. In Sec. V, we derive the renormalized $h V V, h f \bar{f}$ and $h h h$ vertices. For the $h V V$ and $h f \bar{f}$ vertices, we further define the form factors of these vertices. We then show the simple plots for the renormalized scale factors for $h V V$, $h f \bar{f}$, and $h h h$ couplings normalized to their SM predictions and discuss their momentum dependence. Section VI discusses and lists theoretical and experimental constraints to be imposed in the parameter scan of the model. Section VII presents the numerical result for the renormalized scale factors by scanning model parameters under the both theoretical and experimental constraints. Section VIII summarizes our findings in this work. Appendices A and B give explicit formulas of the masses and interactions of the Higgs bosons in the model, respectively. In Appendix C,

\footnotetext{
${ }^{1}$ The custodial symmetry is actually broken explicitly by the $U(1)_{Y}$ gauge coupling as it also happens in the SM.
}

the loop functions are defined, and explicit formulas for contributions from 1PI diagrams that appear in our calculations are presented.

\section{THE MODEL}

The scalar sector of the GM model is composed of a weak isospin doublet field $\phi$ with hypercharge $Y=1 / 2$ and weak isospin triplet fields $\chi$ and $\xi$ with $Y=1$ and $Y=0$, respectively. These scalar fields can be expressed in the $S U(2)_{L} \times S U(2)_{R}$ bi-doublet $(\Phi)$ and bi-triplet $(\Delta)$ forms as

$$
\begin{aligned}
& \Phi \equiv\left(\phi^{c}, \phi\right)=\left(\begin{array}{cc}
\phi^{0 *} & \phi^{+} \\
-\phi^{-} & \phi^{0}
\end{array}\right), \\
& \Delta \equiv\left(\chi^{c}, \xi, \chi\right)=\left(\begin{array}{ccc}
\chi^{0 *} & \xi^{+} & \chi^{++} \\
-\chi^{-} & \xi^{0} & \chi^{+} \\
\chi^{--} & -\xi^{-} & \chi^{0}
\end{array}\right),
\end{aligned}
$$

where $\phi^{c}=i \tau^{2} \phi^{*}$ and $\chi^{c}=C_{3} \chi^{*}$ are the charge-conjugated $\phi$ and $\chi$ fields, respectively. The matrix $C_{3}$ is given by

$$
C_{3}=\left(\begin{array}{ccc}
0 & 0 & 1 \\
0 & -1 & 0 \\
1 & 0 & 0
\end{array}\right) .
$$

The neutral components are parametrized by

$$
\begin{aligned}
\phi^{0} & =\frac{1}{\sqrt{2}}\left(\phi_{r}+v_{\phi}+i \phi_{i}\right), \\
\chi^{0} & =\frac{1}{\sqrt{2}}\left(\chi_{r}+i \chi_{i}\right)+v_{\chi}, \\
\xi^{0} & =\xi_{r}+v_{\xi},
\end{aligned}
$$

where $v_{\phi}, v_{\chi}$ and $v_{\xi}$ are the VEVs of $\phi^{0}, \chi^{0}$ and $\xi^{0}$, respectively. For later convenience, we reexpress the two triplet VEVs by

$$
v_{\chi}=v_{\Delta}, \quad v_{\xi}=v_{\Delta}+\nu .
$$

The $\nu$ parameter describes the deviation from alignment in the triplet VEVs, i.e., $\langle\Delta\rangle=v_{\Delta} \mathbb{1}_{3 \times 3}$.

In the following subsections, we first discuss the scalar potential and explain the necessity of introducing $S U(2)_{L} \times$ $S U(2)_{R}$ breaking terms in order to make the model consistent at loop levels. We then give the Lagrangian of the scalar kinetic terms and the Yukawa interactions. Finally, we discuss the decoupling property of the GM model.

\section{A. Scalar potential}

The $S U(2)_{L} \times U(1)_{Y}$ gauge-invariant scalar potential can be expressed as follows:

$$
V=V_{\text {cust }}(\Phi, \Delta)+V_{\text {chst }}(\phi, \chi, \xi),
$$


where $V_{\text {cust }}$ and $V_{\text {cust }}$ are, respectively, given as a function of $\{\Phi, \Delta\}$ and $\{\phi, \chi, \xi\} .^{2} V_{\text {cust }}$ is defined such that when it is vanishing, the potential has the most general global $S U(2)_{L} \times S U(2)_{R}$ symmetry which is spontaneously broken down to the diagonal part $S U(2)_{V}$, the so-called custodial symmetry, under the assumption of vacuum alignment: $v_{\chi}=v_{\xi}$ or, equivalently, $\nu=0$. In this configuration, the electroweak rho parameter $\rho$ is predicted to be 1 at tree level as we will see in the next subsection.

Nonetheless, even if we take $V_{\text {chst }}=0$ at tree level, $V_{\text {chst }}$ generally re-appears at loop levels due to, e.g., hypercharge gauge boson loops as a consequence of $S U(2)_{L} \times S U(2)_{R}$ breaking effects in the kinetic term. In addition, such loop contributions contain ultra-violet (UV) divergences which cannot be cancelled by counterterms associated with the
$V_{\text {cust }}$ part alone. Therefore, in order to make the model consistent at loop levels, we need to introduce custodial symmetry breaking terms from the beginning. The simplest choice to make our calculations of renormalized vertices for the discovered Higgs boson consistent is to introduce

$$
V_{\text {cust }}=\frac{m_{\xi}^{2}}{2} \xi^{\dagger} \xi,
$$

where $\xi=\left(\xi^{+}, \xi^{0},-\xi^{-}\right)^{T}$. The other possible terms for $V_{\text {cust }}$ can be important for the computation of one-loop corrections to physical quantities related to the extra Higgs bosons, but are not our concerns here.

Explicitly, the most general custodial symmetric potential is given by

$$
\begin{aligned}
V_{\text {cust }}= & m_{\Phi}^{2} \operatorname{tr}\left(\Phi^{\dagger} \Phi\right)+m_{\Delta^{2}}^{2} \operatorname{tr}\left(\Delta^{\dagger} \Delta\right)+\lambda_{1}\left[\operatorname{tr}\left(\Phi^{\dagger} \Phi\right)\right]^{2}+\lambda_{2}\left[\operatorname{tr}\left(\Delta^{\dagger} \Delta\right)\right]^{2}+\lambda_{3} \operatorname{tr}\left[\left(\Delta^{\dagger} \Delta\right)^{2}\right] \\
& +\lambda_{4} \operatorname{tr}\left(\Phi^{\dagger} \Phi\right) \operatorname{tr}\left(\Delta^{\dagger} \Delta\right)+\lambda_{5} \operatorname{tr}\left(\Phi^{\dagger} \frac{\tau^{a}}{2} \Phi \frac{\tau^{b}}{2}\right) \operatorname{tr}\left(\Delta^{\dagger} t^{a} \Delta t^{b}\right) \\
& +\mu_{1} \operatorname{tr}\left(\Phi^{\dagger} \frac{\tau^{a}}{2} \Phi \frac{\tau^{b}}{2}\right)\left(P^{\dagger} \Delta P\right)^{a b}+\mu_{2} \operatorname{tr}\left(\Delta^{\dagger} t^{a} \Delta t^{b}\right)\left(P^{\dagger} \Delta P\right)^{a b},
\end{aligned}
$$

where $\tau^{a} / 2$ and $t^{a}(a=1,2,3)$ are the $2 \times 2$ and $3 \times 3$ representations of the $S U(2)$ generators, respectively. The matrix $P$ gives the similarity transformation $P\left(-i \epsilon^{a}\right) P^{\dagger}=$ $t^{a}$ with $\epsilon^{a}$ being the adjoint representation of the $S U(2)$ generators, and is given as

$$
P=\left(\begin{array}{ccc}
-1 / \sqrt{2} & i / \sqrt{2} & 0 \\
0 & 0 & 1 \\
1 / \sqrt{2} & i / \sqrt{2} & 0
\end{array}\right) .
$$

To obtain the mass eigenvalues for the physical Higgs bosons, one imposes the tadpole conditions at tree level:

$$
\left.\frac{\partial V}{\partial \phi_{r}}\right|_{0}=\left.\frac{\partial V}{\partial \chi_{r}}\right|_{0}=\left.\frac{\partial V}{\partial \xi_{r}}\right|_{0}=0
$$

Using the above three equations, one can re-write the three mass parameters $m_{\Phi}^{2}, m_{\Delta}^{2}$ and $m_{\xi}^{2}$ in terms of the other parameters in the scalar potential. We note that in the limit of $\nu=0, m_{\xi}^{2}$ also vanishes and the tadpole conditions for $\chi_{r}$ and $\xi_{r}$ become identical as a consequence of restoring the custodial symmetry at tree level. Detailed

\footnotetext{
${ }^{2}$ Given the scalar fields in the model, the most general $S U(2)_{L} \times U(1)_{Y}$ gauge-invariant scalar potential has 14 real and 2 complex parameters. Imposing the global $S U(2)_{L} \times$ $S U(2)_{R}$ symmetry renders relations among the parameters and results in the custodial symmetric potential given in Eq. (7) described by nine real parameters, as shown in Ref. [28].
}

analytic expressions for the physical Higgs bosons and their squared masses are presented in Appendix A for the general $\nu \neq 0$ case.

We here highlight some important properties of the mass spectrum in the $\nu=0$ limit. The mass eigenstates of Higgs bosons can be classified under the custodial $S U(2)_{V}$ symmetry into one 5-plet $\left(H_{5}^{ \pm \pm}, H_{5}^{ \pm}, H_{5}^{0}\right)$, one 3-plet $\left(H_{3}^{ \pm}, H_{3}^{0}\right)$ and two singlets $H_{1}$ and $h$ with $h$ being identified with the observed $125 \mathrm{GeV}$ Higgs boson in our work. The Higgs bosons belonging to the same $S U(2)_{V}$ multiplet are degenerate in mass, as seen in Eq. (A4). Taking $\nu \rightarrow 0$, various mixing angles defined in Eq. (A1) among the scalar bosons reduce to

$$
\begin{aligned}
\tan \beta & \equiv \tan \beta_{\text {odd }}=\tan \beta_{1}^{ \pm}=\frac{v_{\phi}}{2 \sqrt{2} v_{\Delta}}, \\
\tan \beta_{2}^{ \pm} & =\tan \gamma=\tan \alpha_{1,2}=0, \\
\tan \alpha & \equiv \tan \alpha_{3} .
\end{aligned}
$$

Therefore, the rotation matrices to separate the NambuGoldstone (NG) bosons from the physical CP-odd and singly charged Higgs bosons become the same. This also shows the recovery of the custodial symmetry. Consequently, all the potential parameters can be expressed in terms of the following nine parameters:

$$
m_{H_{5}}^{2}, m_{H_{3}}^{2}, m_{H_{1}}^{2}, m_{h}^{2}, \mu_{1}, \mu_{2}, v, \beta, \alpha .
$$




\section{B. Kinetic terms}

The kinetic terms of the scalar fields are given by

$$
\mathcal{L}_{\text {kin }}=\frac{1}{2} \operatorname{tr}\left(D_{\mu} \Phi\right)^{\dagger}\left(D^{\mu} \Phi\right)+\frac{1}{2} \operatorname{tr}\left(D_{\mu} \Delta\right)^{\dagger}\left(D^{\mu} \Delta\right),
$$

where the covariant derivatives

$$
\begin{aligned}
& D_{\mu} \Phi=\partial_{\mu} \Phi-i g \frac{\tau^{a}}{2} W_{\mu}^{a} \Phi+i g^{\prime} B_{\mu} \Phi \frac{\tau^{3}}{2}, \\
& D_{\mu} \Delta=\partial_{\mu} \Delta-i g t^{a} W_{\mu}^{a} \Delta+i g^{\prime} B_{\mu} \Delta t^{3} .
\end{aligned}
$$

The weak gauge boson masses are calculated to be

$$
m_{W}^{2}=\frac{g^{2}}{4}\left(v_{\phi}^{2}+8 v_{\Delta}^{2}+\bar{\nu}^{2}\right), \quad m_{Z}^{2}=\frac{g_{Z}^{2}}{4}\left(v_{\phi}^{2}+8 v_{\Delta}^{2}\right),
$$

where $\bar{\nu} \equiv 2 \sqrt{\nu\left(2 v_{\Delta}+\nu\right)}$ and $g_{Z} \equiv \sqrt{g^{2}+g^{\prime 2}}$. As in the $\mathrm{SM}$, the electroweak symmetry breaking $S U(2)_{L} \times$ $U(1)_{Y} \rightarrow U(1)_{\mathrm{EM}}$ forces the following relation among the gauge couplings:

$$
e\left(=\sqrt{4 \pi \alpha_{\mathrm{EM}}}\right)=g s_{W}=g^{\prime} c_{W},
$$

where $\alpha_{\mathrm{EM}}$ is the fine structure constant and $c_{W}\left(s_{W}\right)$ is the cosine (sine) of the weak mixing angle $\theta_{W}$. Using these relations, we can also write $g_{Z}=g / c_{W}$. From Eq. (14), we can identify the VEV $v$, which is related to the Fermi's decay constant $G_{F}$ by $v=\left(\sqrt{2} G_{F}\right)^{-1 / 2}$, as

$$
v^{2}=v_{\phi}^{2}+8 v_{\Delta}^{2}+\bar{\nu}^{2} .
$$

The tree-level rho parameter is then given by

$$
\rho_{\text {tree }} \equiv \frac{m_{W}^{2}}{m_{Z}^{2} c_{W}^{2}}=\frac{v^{2}}{v^{2}-\bar{\nu}^{2}} .
$$

Therefore, a nonzero $\nu$ would make the rho parameter deviate from unity at tree level. This implies that unlike in the SM, the electroweak sector is now empirically fixed by four independent parameters, e.g., the set of $\left\{m_{W}, m_{Z}, \alpha_{\mathrm{EM}}, \nu\right\}$. In fact, the necessity of four input parameters in the electroweak sector generally appears in models with $\rho_{\text {tree }} \neq 1[29,30]$. In terms of these four parameters, $s_{W}^{2}$ and $v^{2}$ are given by

$$
s_{W}^{2}=1-\frac{m_{W}^{2}}{m_{Z}^{2}}\left(1-\frac{\bar{\nu}^{2}}{v^{2}}\right), \quad v^{2}=\frac{m_{W}^{2} s_{W}^{2}}{\pi \alpha_{\mathrm{EM}}} .
$$

The three- and four-point interaction terms of Higgs bosons to gauge bosons are also obtained from Eq. (13), with their expressions in the $\nu \rightarrow 0$ limit given in Appendix B. We here list several remarkable features regarding the gauge interactions of the Higgs bosons in the model:
(1) The SM-like Higgs boson couplings $h W W$ and $h Z Z$ can be larger than the SM predictions at tree level. This does not happen in models constructed with only singlet and/or doublet scalars.

(2) The 5-plet Higgs bosons have the scalar-gaugegauge type interactions, while the 3-plet Higgs bosons do not, as seen in Eq. (B1). The 3-plet Higgs bosons are thus said to be gauge-phobic.

(3) The $H_{5}^{0} W W$ coupling normalized by the SM $h W W$ coupling defined as $c_{H_{5} W W}$ is different from that associated with the $Z$ boson (i.e., $c_{H_{5} Z Z}$ ). In particular, $c_{H_{5} Z Z} / c_{H_{5} W W}=-2$, as seen in Eq. (B2). This property is not seen in the corresponding couplings of $h$ and $H_{1}$.

\section{Yukawa interactions}

The Yukawa Lagrangian for the third-generation fermions is given by

$\mathcal{L}_{Y}=-y_{t} \bar{Q}_{L}^{3} \phi^{c} t_{R}-y_{b} \bar{Q}_{L}^{3} \phi b_{R}-y_{\tau} \bar{L}_{L}^{3} \phi \tau_{R}+$ H.c.,

where $Q_{L}^{3}=(t, b)_{L}^{T}$ and $L_{L}^{3}=\left(\nu_{\tau}, \tau\right)_{L}^{T}$. The Yukawa interactions for the other SM fermions can be simply obtained by generalizing the above Yukawa couplings to $3 \times 3$ Yukawa matrices. In the $\nu \rightarrow 0$ limit, fermion masses are obtained as $m_{f}=y_{f} v s_{\beta} / \sqrt{2}$ for $f \in\{t, b, \tau\}$. We note that there is another type of Yukawa interaction terms for the $\chi$ field, which is expressed as

$$
\overline{L_{L}^{c}} i \tau^{2} \chi L_{L}+\text { H.c. }
$$

and gives Majorana mass to left-handed neutrinos. Typically, the size of this Yukawa coupling is expected to be as small as $\mathcal{O}\left(10^{-9}-10^{-10}\right)$ for $v_{\Delta}=\mathcal{O}(1) \mathrm{GeV}$ to reproduce the observed neutrino oscillations. Thus, these interactions do not play any important role in the following discussions and are ignored throughout this paper.

The interaction terms for the physical Higgs bosons are given in Appendix B in the $\nu \rightarrow 0$ limit. It should be noted that the 5-plet Higgs bosons do not couple to fermions and are thus fermio-phobic, while the 3-plet Higgs bosons do. In fact, the structure of the Yukawa couplings of the 3-plet Higgs bosons is the same as that of the CP-odd and charged Higgs bosons in the Type-I THDM.

\section{Decoupling limit}

In this subsection, we briefly discuss the decoupling limit in the GM model. As clarified in Ref. [31], the decoupling limit can be realized by taking $m_{\Delta}$ to infinity, with all the extra Higgs boson masses also going to infinity and the SM predictions being reproduced.

In order to clearly see how the decoupling limit works, we expand physical parameters in the decoupling regime 
(i.e., $m_{\Delta} \gg v$ ) in powers of $1 / m_{\Delta}^{2}$. The masses of extra Higgs bosons are expanded as

$$
\begin{aligned}
& m_{H_{5}}=\frac{m_{\Delta}}{4 \sqrt{2}}\left[8+\frac{v^{2}}{m_{\Delta}^{2}}\left(4 \lambda_{4}-\lambda_{5}\right)\right]+\mathcal{O}\left(\frac{v^{4}}{m_{\Delta}^{3}}\right), \\
& m_{H_{3}}=\frac{m_{\Delta}}{4 \sqrt{2}}\left[8+\frac{v^{2}}{m_{\Delta}^{2}}\left(4 \lambda_{4}+\lambda_{5}\right)\right]+\mathcal{O}\left(\frac{v^{4}}{m_{\Delta}^{3}}\right), \\
& m_{H_{1}}=\frac{m_{\Delta}}{4 \sqrt{2}}\left[8+\frac{v^{2}}{m_{\Delta}^{2}}\left(4 \lambda_{4}+2 \lambda_{5}\right)\right]+\mathcal{O}\left(\frac{v^{4}}{m_{\Delta}^{3}}\right) .
\end{aligned}
$$

With these mass parameters growing virtually linearly with $m_{\Delta}$, these extra Higgs bosons are decoupled from the theory in the $m_{\Delta} \rightarrow \infty$ limit. It is also seen that the differences among these mass parameters are suppressed by $\mathcal{O}\left(1 / m_{\Delta}\right)$ or higher. Keeping terms up to order $1 / m_{\Delta}$, we obtain a relation among these mass parameters [32]

$$
m_{H_{1}}=\frac{3}{2} m_{H_{3}}-\frac{1}{2} m_{H_{5}} .
$$

On the other hand, the mass of the SM-like Higgs boson $h$ is mainly given by the $\lambda_{1}$ term as in the SM:

$$
\begin{aligned}
\lambda_{1}= & \frac{1}{8}\left\{\frac{m_{h}^{2}}{v^{2}}+\frac{3 \mu_{1}^{2}}{8 m_{\Delta}^{2}}+\frac{\mu_{1}^{2}}{16 m_{\Delta}^{4}}\left[5 m_{h}^{2}-9 v^{2}\left(2 \lambda_{4}+\lambda_{5}\right)\right]\right\} \\
& +\mathcal{O}\left(\frac{v^{6}}{m_{\Delta}^{6}}\right) .
\end{aligned}
$$

Next, we check the decoupling behavior of the couplings associated with the SM-like Higgs boson $h$. At tree level, the $h$ couplings are modified from the SM predictions due to the mixing between the CP-even Higgs bosons and the VEVs. The former and the latter are, respectively, parametrized by $\alpha$ and $v_{\Delta}$ (or $\beta$ ). Expanding in powers of $1 / m_{\Delta}^{2}$, we obtain

$$
\begin{aligned}
\sin \alpha= & -\frac{\sqrt{3} v\left|\mu_{1}\right|}{8 m_{\Delta}^{2}}\left\{2+\frac{1}{m_{\Delta}^{2}}\left[m_{h}^{2}-2 v^{2}\left(2 \lambda_{4}+\lambda_{5}\right)\right]\right\} \\
& +\mathcal{O}\left(\frac{v^{6}}{m_{\Delta}^{6}}\right), \\
v_{\Delta}= & \frac{v^{2}\left|\mu_{1}\right|}{16 m_{\Delta}^{2}}\left[2-\frac{v^{2}}{m_{\Delta}^{2}}\left(2 \lambda_{4}+\lambda_{5}\right)\right]+\mathcal{O}\left(\frac{v^{5}}{m_{\Delta}^{4}}\right) .
\end{aligned}
$$

As expected, both of these parameters approach zero in the limit of $m_{\Delta} \rightarrow \infty$ with $\mu_{1}$ taken to be finite. The decoupling behavior of the $h$ couplings can be shown more directly by expanding the normalized $h V V\left(c_{h V V}\right), h f f\left(c_{h f f}\right)$ and $h h h\left(c_{h h h}\right)$ couplings by their SM values as

$$
\begin{aligned}
c_{h V V} & =1+\frac{3 v^{2}}{32} \frac{\mu_{1}^{2}}{m_{\Delta}^{4}}+\mathcal{O}\left(\frac{v^{6}}{m_{\Delta}^{6}}\right), \\
c_{h f f} & =1-\frac{v^{2}}{32} \frac{\mu_{1}^{2}}{m_{\Delta}^{4}}+\mathcal{O}\left(\frac{v^{6}}{m_{\Delta}^{6}}\right), \\
c_{h h h} & =1+\frac{v^{2} \mu_{1}^{2}}{32 m_{\Delta}^{4}}\left[\frac{12 v^{2}}{m_{h}^{2}}\left(2 \lambda_{4}+\lambda_{5}\right)-7\right]+\mathcal{O}\left(\frac{v^{6}}{m_{\Delta}^{6}}\right) .
\end{aligned}
$$

As expected, they all become one in the decoupling limit.

\section{RENORMALIZATION}

In this section, we discuss the renormalization prescription to obtain finite one-loop corrected Higgs boson couplings. Our renormalization is based on the on-shell scheme, where counterterms are introduced to cancel the radiative corrections to the mass parameters (as well as wave functions) for various fields on their mass shells.

In our calculation, unrenormalized one-loop contributions to two-point and three-point functions are constructed in the so-called tadpole scheme $[33,34]$ as

$$
\begin{aligned}
\Pi_{A B}\left(p^{2}\right) & =\Pi_{A B}^{1 \mathrm{PI}}\left(p^{2}\right)+\Pi_{A B}^{\mathrm{Tad}} \quad \text { (for two-point functions), } \\
\Gamma_{A B C}\left(p_{1}^{2}, p_{2}^{2}, q^{2}\right) & =\Gamma_{A B C}^{1 \mathrm{PI}}\left(p_{1}^{2}, p_{2}^{2}, q^{2}\right)+\Gamma_{A B C}^{\mathrm{Tad}} \quad \text { (for three-point functions), }
\end{aligned}
$$

where $A, B$, and $C$ refer to particles on the external legs. The first and second terms on the right-hand sides denote the contributions from one-particle irreducible (1PI) and tadpole inserted diagrams, respectively. Obviously, there is no momentum dependence in the tadpole inserted contributions $\left(\Pi_{A B}^{\mathrm{Tad}}\right.$ and $\left.\Gamma_{A B C}^{\mathrm{Tad}}\right)$. For later convenience, we define the derivative

$$
\left.\Pi_{A B}^{\prime}\left(m^{2}\right) \equiv \frac{d}{d p^{2}} \Pi_{A B}\left(p^{2}\right)\right|_{p^{2}=m^{2}}
$$

for a generic two-point function. We assume that effects of custodial $S U(2)_{V}$ symmetry breaking are introduced at the one-loop level; namely, we take the $S U(2)_{V}$ breaking parameter $\nu=0$ at tree level. Therefore, in the calculations of one-loop diagrams, we can make use of the tree-level properties discussed in the previous section, such as a degenerate mass for the Higgs bosons belonging to the same $S U(2)_{V}$ multiplet, because including deviations from the tree-level properties would be of higher-order corrections.

In the subsequent subsections, we discuss the renormalization of the parameters in the gauge sector, the fermion sector and the scalar sector in order. 


\section{A. Gauge sector}

We shift the following electroweak parameters and the field wave functions of $S U(2)_{L}$ and $U(1)_{Y}$ gauge bosons denoted by $W_{\mu}^{a}(a=1,2,3)$ and $B_{\mu}$ as:

$$
\begin{aligned}
& m_{W}^{2} \rightarrow m_{W}^{2}+\delta m_{W}^{2}, \quad m_{Z}^{2} \rightarrow m_{Z}^{2}+\delta m_{Z}^{2}, \quad \alpha_{\mathrm{EM}} \rightarrow \alpha_{\mathrm{EM}}+\delta \alpha_{\mathrm{EM}}, \quad \nu \rightarrow 0+\delta \nu, \\
& W_{\mu}^{a} \rightarrow\left(1+\frac{1}{2} \delta Z_{W}\right) W_{\mu}^{a}, \quad B_{\mu} \rightarrow\left(1+\frac{1}{2} \delta Z_{B}\right) B_{\mu},
\end{aligned}
$$

in which we have introduced six counterterms. Using Eq. (18), the counterterms $\delta v$ and $\delta s_{W}^{2}$ are given by

$$
\begin{aligned}
\delta s_{W}^{2} & =-\frac{m_{W}^{2}}{m_{Z}^{2}}\left(\frac{\delta m_{W}^{2}}{m_{W}^{2}}-\frac{\delta m_{Z}^{2}}{m_{Z}^{2}}-\frac{8 v_{\Delta} \delta \nu}{v^{2}}\right), \\
\frac{\delta v}{v} & =\frac{1}{2}\left[\left(1-\frac{c_{W}^{2}}{s_{W}^{2}}\right) \frac{\delta m_{W}^{2}}{m_{W}^{2}}+\frac{c_{W}^{2}}{s_{W}^{2}} \frac{\delta m_{Z}^{2}}{m_{Z}^{2}}-\frac{\delta \alpha_{\mathrm{EM}}}{\alpha_{\mathrm{EM}}}+\frac{c_{W}^{2}}{s_{W}^{2}} \frac{8 v_{\Delta} \delta \nu}{v^{2}}\right] .
\end{aligned}
$$

Furthermore, it is convenient to define the following counterterms for the wave functions of the physical $Z$ boson and photon fields:

$$
\begin{aligned}
\left(\begin{array}{c}
\delta Z_{Z} \\
\delta Z_{\gamma}
\end{array}\right) & =\left(\begin{array}{ll}
c_{W}^{2} & s_{W}^{2} \\
s_{W}^{2} & c_{W}^{2}
\end{array}\right)\left(\begin{array}{c}
\delta Z_{W} \\
\delta Z_{B}
\end{array}\right), \\
\delta Z_{Z \gamma} & =\frac{c_{W} s_{W}}{c_{W}^{2}-s_{W}^{2}}\left(\delta Z_{Z}-\delta Z_{\gamma}\right) .
\end{aligned}
$$

The renormalized gauge boson two-point functions $\hat{\Pi}_{X Y},(X Y=W W, Z Z, Z \gamma, \gamma \gamma)$ can then be defined as follows,

$$
\begin{aligned}
\hat{\Pi}_{W W}\left(p^{2}\right) & =\Pi_{W W}\left(p^{2}\right)-\delta m_{W}^{2}+\delta Z_{W}\left(p^{2}-m_{W}^{2}\right), \\
\hat{\Pi}_{Z Z}\left(p^{2}\right) & =\Pi_{Z Z}\left(p^{2}\right)-\delta m_{Z}^{2}+\delta Z_{Z}\left(p^{2}-m_{Z}^{2}\right), \\
\hat{\Pi}_{Z \gamma}\left(p^{2}\right) & =\Pi_{Z \gamma}\left(p^{2}\right)+\delta Z_{Z \gamma}\left(p^{2}-\frac{m_{Z}^{2}}{2}\right)-m_{Z}^{2} \frac{\delta s_{W}^{2}}{2 s_{W} c_{W}}, \\
\hat{\Pi}_{\gamma \gamma}\left(p^{2}\right) & =\Pi_{\gamma \gamma}\left(p^{2}\right)+p^{2} \delta Z_{\gamma},
\end{aligned}
$$

with $\Pi_{X Y}$ being the unrenormalized two-point functions defined in Eq. (26).

In order to determine the counterterms in Eq. (28), we impose the following five on-shell conditions, which are the same as those used in the SM [35]:

$$
\begin{aligned}
& \operatorname{Re} \hat{\Pi}_{W W}\left(m_{W}^{2}\right)=0, \quad \operatorname{Re}_{Z Z}\left(m_{Z}^{2}\right)=0, \quad \hat{\Pi}_{\gamma \gamma}{ }^{\prime}(0)=0, \quad \hat{\Pi}_{Z \gamma}(0)=0, \\
& \hat{\Gamma}_{\mu}^{\gamma e e}\left(q^{2}=0, \not p_{1}=\not p_{2}=m_{e}\right)=i e \gamma_{\mu},
\end{aligned}
$$

where $\hat{\Gamma}_{\mu}^{\gamma e e}$ is the renormalized photon-electron-positron vertex. From them, the five counterterms are determined as follows:

$$
\begin{aligned}
\delta m_{W}^{2} & =\operatorname{Re} \Pi_{W W}\left(m_{W}^{2}\right), \quad \delta m_{Z}^{2}=\operatorname{Re} \Pi_{Z Z}\left(m_{Z}^{2}\right), \quad \frac{\delta \alpha_{\mathrm{EM}}}{\alpha_{\mathrm{EM}}}=\Pi_{\gamma \gamma}^{\prime}(0)+\frac{2 s_{W}}{c_{W}} \frac{\Pi_{Z \gamma}(0)}{m_{Z}^{2}}, \\
\delta Z_{\gamma} & =-\Pi_{\gamma \gamma}^{\prime}(0), \quad \delta Z_{Z \gamma}=\frac{2}{m_{Z}^{2}} \Pi_{Z \gamma}(0)+\frac{\delta s_{W}^{2}}{s_{W} c_{W}}
\end{aligned}
$$

Using Eq. (30), one then finds 
$\delta Z_{Z}=-\Pi_{\gamma \gamma}^{\prime}(0)+\frac{2\left(c_{W}^{2}-s_{W}^{2}\right)}{c_{W} s_{W}} \frac{\Pi_{Z \gamma}(0)}{m_{Z}^{2}}+\frac{c_{W}^{2}-s_{W}^{2}}{c_{W}^{2}} \frac{\delta s_{W}^{2}}{s_{W}^{2}}$,

$\delta Z_{W}=-\Pi_{\gamma \gamma}^{\prime}(0)+\frac{2 c_{W}}{s_{W}} \frac{\Pi_{Z \gamma}(0)}{m_{Z}^{2}}+\frac{\delta s_{W}^{2}}{s_{W}^{2}}$.

As explained in Sec. II, there is one additional counterterm $\delta \nu$ in the GM model. Therefore, we need another condition to fix it. Following the earlier work in Ref. [25], we demand that the electroweak oblique $T$ parameter, $T \equiv T_{\mathrm{GM}}-T_{\mathrm{SM}}$ with $T_{\mathrm{GM}}$ and $T_{\mathrm{SM}}$ being, respectively, the $T$ parameter calculated in the GM model and the SM, be equal to its experimental value,

$$
T=T_{\text {exp }},
$$

where

$$
\alpha_{\mathrm{EM}} T=\frac{\Delta \Pi_{Z Z}(0)}{m_{Z}^{2}}-\frac{\Delta \Pi_{W W}(0)}{m_{W}^{2}}+\frac{8 v_{\Delta} \delta \nu}{v^{2}},
$$

with $\left.\Delta \Pi_{V V} \equiv \Pi_{V V}\right|_{\mathrm{GM}}-\left.\Pi_{V V}\right|_{\mathrm{SM}}$. Therefore, $\delta \nu$ is determined as

$$
\delta \nu=\frac{v^{2}}{8 v_{\Delta}}\left[\frac{\Delta \Pi_{W W}(0)}{m_{W}^{2}}-\frac{\Delta \Pi_{Z Z}(0)}{m_{Z}^{2}}+\alpha_{\mathrm{EM}} T_{\exp }\right] .
$$

We will set $T_{\exp }=0$ in the discussion of numerical analyses.

\section{B. Fermion sector}

The renormalization for the fermion sector can be done in the same way as in the SM. Left-handed and righthanded fermions $\left(\psi_{L}\right.$ and $\left.\psi_{R}\right)$ and their masses $m_{f}$ are shifted as

$\psi_{L / R} \rightarrow\left(1+\frac{1}{2} \delta Z_{L / R}^{f}\right) \psi_{L / R}, \quad m_{f} \rightarrow m_{f}+\delta m_{f}$.

Following Ref. [35], these counterterms are given by

$$
\begin{aligned}
& \delta m_{f}=m_{f}\left[\Pi_{f f, V}\left(m_{f}^{2}\right)+\Pi_{f f, S}\left(m_{f}^{2}\right)\right], \\
& \delta Z_{V}^{f}\left(\equiv \frac{\delta Z_{L}^{f}+\delta Z_{R}^{f}}{2}\right)=-\Pi_{f f, V}\left(m_{f}^{2}\right)-2 m_{f}^{2}\left[\Pi_{f f, V}^{\prime}\left(m_{f}^{2}\right)+\Pi_{f f, S}^{\prime}\left(m_{f}^{2}\right)\right],
\end{aligned}
$$

where $\Pi_{f f, V}$ and $\Pi_{f f, S}$ are the vector and scalar parts of the fermion two-point functions defined in Eq. (C21) at the one-loop level, respectively. Although another independent wave function renormalization factor $\delta Z_{A}^{f}=\left(\delta Z_{L}^{f}-\delta Z_{R}^{f}\right) / 2$ can be constructed, it does not appear in subsequent discussions.

\section{Scalar sector}

Finally, we discuss the renormalization of parameters in the scalar potential. In particular, we concentrate on the neutral scalar part, because the charged scalar states are not relevant for the discussions of the renormalized Higgs boson vertices in Sec. V. We shift the parameters defined in Eq. (11) as follows,

$$
\begin{aligned}
& \left(m_{H_{5}}^{2}, m_{H_{3}}^{2}, m_{H_{1}}^{2}, m_{h}^{2}\right) \rightarrow\left(m_{H_{5}}^{2}, m_{H_{3}}^{2}, m_{H_{1}}^{2}, m_{h}^{2}\right)+\left(\delta m_{H_{5}}^{2}, \delta m_{H_{3}}^{2}, \delta m_{H_{1}}^{2}, \delta m_{h}^{2}\right), \\
& \mu_{i} \rightarrow \mu_{1,2}+\delta \mu_{1,2}, \quad \beta \rightarrow \beta+\delta \beta, \quad \alpha \rightarrow \alpha+\delta \alpha, \quad \alpha_{1,2} \rightarrow 0+\delta \alpha_{1,2},
\end{aligned}
$$

where the shifts for $v$ and $\nu$ are already done in Sec. III A. We here also shift the mixing angles $\alpha_{1}$ and $\alpha_{2}$ which become zero at tree level due to the custodial symmetry. ${ }^{3}$ We note that there are also counterterms for the three tadpoles of $\phi_{r}, \chi_{r}$, and $\xi_{r}$. But these counterterms should be zero in the tadpole scheme [34], as their contributions are already included in the tadpole inserted diagrams in Eq. (26). The wave functions for the CP-odd and CP-even Higgs bosons are then shifted as follows:

$$
\begin{aligned}
&\left(\begin{array}{c}
G^{0} \\
H_{3}^{0}
\end{array}\right) \rightarrow\left[1+\frac{1}{2}\left(\begin{array}{cc}
\delta Z_{G^{0}} & \delta Z_{G^{0} H_{3}^{0}}+2 \delta \beta \\
\delta Z_{H_{3}^{0} G^{0}}-2 \delta \beta & \delta Z_{H_{3}^{0}}
\end{array}\right)\right]\left(\begin{array}{c}
G^{0} \\
H_{3}^{0}
\end{array}\right), \\
&\left(\begin{array}{c}
H_{1} \\
h \\
H_{5}^{0}
\end{array}\right) \rightarrow\left[1+\frac{1}{2}\left(\begin{array}{ccc}
\delta Z_{H_{1}} & \delta Z_{H_{1} h}+2 \delta \alpha & \delta Z_{H_{1} H_{5}^{0}}+2 \delta \alpha_{2} \\
\delta Z_{h H_{1}}-2 \delta \alpha & \delta Z_{h} & \delta Z_{h H_{5}^{0}}+2 \delta \alpha_{1} \\
\delta Z_{H_{5}^{0} H_{1}}-2 \delta \alpha_{2} & \delta Z_{H_{5}^{0} h}-2 \delta \alpha_{1} & \delta Z_{H_{5}^{0}}
\end{array}\right)\right]\left(\begin{array}{c}
H_{1} \\
h \\
H_{5}^{0}
\end{array}\right),
\end{aligned}
$$

where $\delta Z_{i j}=\delta Z_{j i}$.

\footnotetext{
${ }^{3}$ In our choice of the scalar potential given in Eqs. (6) and (7), the counterterms $\delta \alpha_{1,2}$ can be expressed by the other counterterms given in Eq. (40).
} 
The renormalized two-point functions for the neutral scalar fields are given by

$$
\begin{aligned}
\hat{\Pi}_{S S^{\prime}}\left(p^{2}\right)= & \Pi_{S S^{\prime}}\left(p^{2}\right)+\frac{1}{2}\left(2 p^{2}-m_{S}^{2}-m_{S^{\prime}}^{2}\right) \delta Z_{S S^{\prime}} \\
& -\left(m_{S}^{2}-m_{S^{\prime}}^{2}\right) \delta \theta_{S S^{\prime}},
\end{aligned}
$$

where $S, S^{\prime} \in\left\{H_{5}^{0}, H_{1}, h, H_{3}^{0}, G^{0}\right\}, \quad \delta Z_{S S}=\delta Z_{S} \quad$ and $\left(m_{H_{5}^{0}}^{2}, m_{H_{3}^{0}}^{2}, m_{G^{0}}^{2}\right)=\left(m_{H_{5}}^{2}, m_{H_{3}}^{2}, 0\right)$. In addition, $\delta \theta_{S S^{\prime}}$ is $\delta \alpha, \delta \alpha_{1}, \delta \alpha_{2}$ and $\delta \beta$ for $\left(S, S^{\prime}\right)=\left(H_{1}, h\right),\left(h, H_{5}^{0}\right)$, $\left(H_{1}, H_{5}^{0}\right)$ and $\left(G^{0}, H_{3}^{0}\right)$, respectively, and $\delta \theta_{S S^{\prime}}=-\delta \theta_{S^{\prime} S}$. To determine these counterterms, we impose the following on-shell conditions:

$$
\begin{aligned}
\operatorname{Re} \hat{\Pi}_{S S}\left(m_{S}^{2}\right) & =0, \quad \operatorname{Re} \hat{\Pi}_{S S}^{\prime}\left(m_{S}^{2}\right)=0, \\
\operatorname{Re} \hat{\Pi}_{S S^{\prime}}\left(m_{S}^{2}\right) & =\operatorname{Re} \hat{\Pi}_{S S^{\prime}}\left(m_{S^{\prime}}^{2}\right)=0 \quad \text { for } S \neq S^{\prime} .
\end{aligned}
$$

Counterterms are then determined as

$$
\delta m_{S}^{2}=\operatorname{Re} \Pi_{S S}\left(m_{S}^{2}\right), \quad \delta Z_{S}=-\operatorname{Re} \Pi_{S S}^{\prime}\left(m_{S}^{2}\right),
$$

and

$$
\begin{aligned}
\delta \alpha & =\frac{1}{2\left(m_{H_{1}}^{2}-m_{h}^{2}\right)} \operatorname{Re}\left[\Pi_{H_{1} h}\left(m_{H_{1}}^{2}\right)+\Pi_{H_{1} h}\left(m_{h}^{2}\right)\right], \\
\delta \alpha_{1} & =\frac{1}{2\left(m_{h}^{2}-m_{H_{5}}^{2}\right)} \operatorname{Re}\left[\Pi_{H_{5}^{0} h}\left(m_{h}^{2}\right)+\Pi_{H_{5}^{0} h}\left(m_{H_{5}}^{2}\right)\right], \\
\delta \alpha_{2} & =\frac{1}{2\left(m_{H_{1}}^{2}-m_{H_{5}}^{2}\right)} \operatorname{Re}\left[\Pi_{H_{1} H_{5}^{0}}\left(m_{H_{1}}^{2}\right)+\Pi_{H_{1} H_{5}^{0}}\left(m_{H_{5}}^{2}\right)\right], \\
\delta \beta & =-\frac{1}{2 m_{H_{3}}^{2}} \operatorname{Re}\left[\Pi_{G^{0} H_{3}^{0}}(0)+\Pi_{G^{0} H_{3}^{0}}\left(m_{H_{3}}^{2}\right)\right], \\
\delta Z_{H_{1} h} & =-\frac{1}{m_{H_{1}}^{2}-m_{h}^{2}} \operatorname{Re}\left[\Pi_{H_{1} h}\left(m_{H_{1}}^{2}\right)-\Pi_{H_{1} h}\left(m_{h}^{2}\right)\right], \\
\delta Z_{h H_{5}^{0}} & =-\frac{1}{m_{h}^{2}-m_{H_{5}}^{2}} \operatorname{Re}\left[\Pi_{H_{5}^{0} h}\left(m_{h}^{2}\right)-\Pi_{H_{5}^{0} h}\left(m_{H_{5}}^{2}\right)\right], \\
\delta Z_{H_{1} H_{5}^{0}} & =-\frac{1}{m_{H_{1}}^{2}-m_{H_{5}}^{2}} \operatorname{Re}\left[\Pi_{H_{1} H_{5}^{0}}\left(m_{H_{1}}^{2}\right)-\Pi_{H_{1} H_{5}^{0}}\left(m_{H_{5}}^{2}\right)\right], \\
\delta Z_{G^{0} H_{3}^{0}} & =\frac{1}{m_{H_{3}}^{2}} \operatorname{Re}\left[\Pi_{G^{0} H_{3}^{0}}(0)-\Pi_{G^{0} H_{3}^{0}}\left(m_{H_{3}}^{2}\right)\right] .
\end{aligned}
$$

There are still two counterterms $\delta \mu_{1,2}$ that are not fixed by the above conditions. These counterterms appear in the renormalized $h h h$ vertex, and we will discuss how to determine these counterterms in Sec. V C.

\section{GAUGE DEPENDENCE}

In the previous section, we have determined all the counterterms by imposing the on-shell renormalization conditions except for $\delta \mu_{1,2}$. As a result, they can be expressed in terms of two-point functions defined in Eq. (26). However, it has been known that there remains gauge dependence in the counterterms for the mixing angles, e.g., $\delta \beta$ and $\delta \alpha$, in the on-shell scheme as it can be proved using the Nielsen identity [36].

In this section, we first show the gauge dependence in the scalar two-point functions, particularly for the CP-even and CP-odd scalar bosons. In order to manifestly show the gauge dependence, we perform the calculation in the general $R_{\xi}$ gauge, where the propagator of a gauge boson $V(=W, Z)$ is expressed using the gauge parameter $\xi_{V}$ as

$$
\frac{-i}{p^{2}-m_{V}^{2}}\left[g^{\mu \nu}-\left(1-\xi_{V}\right) \frac{p^{\mu} p^{\nu}}{p^{2}-\xi_{V} m_{V}^{2}}\right] .
$$

We then discuss how one can remove such gauge dependence by employing the pinch technique $[37,38]$.

\section{A. CP-even part}

First, we show explicitly the cancellation of the gauge dependence in the mixing of CP-even Higgs bosons. Here, we only show the $\xi_{W}$-dependent part because the $\xi_{Z}$ part can be simply obtained by the replacements of $\left(g, W, G^{ \pm}\right) \rightarrow\left(g_{Z} / 2, Z, G^{0}\right)$. The two-point functions for $\phi_{1}-\phi_{2}\left(\phi_{1,2} \in\left\{h, H_{1}\right\}\right)$ are expressed as

$$
\Pi_{\phi_{1} \phi_{2}}\left(q^{2}\right)=\left.\Pi_{\phi_{1} \phi_{2}}\left(q^{2}\right)\right|_{\xi=1}+\left.\Pi_{\phi_{1} \phi_{2}}\left(q^{2}\right)\right|_{\text {G.D. }},
$$

where the first term on the right-hand side corresponds to the result calculated in the 't Hooft-Feynman gauge. On the other hand, the second term in Eq. (47) depends on the gauge parameter and is explicitly given by

$$
\begin{aligned}
\left.\Pi_{\phi_{1} \phi_{2}}\left(q^{2}\right)\right|_{\text {G.D. }}= & \left(1-\xi_{W}\right) \frac{g^{2}}{64 \pi^{2}}\left[c_{\phi_{1} V V} c_{\phi_{2} V V}\left(q^{4}-m_{\phi_{1}}^{2} m_{\phi_{2}}^{2}\right) C_{0}\left(q^{2} ; W, G^{ \pm}\right)\right. \\
& +2 c_{\phi_{1} H_{3} V} c_{\phi_{2} H_{3} V} f\left(q^{2} ; m_{\phi_{1}}, m_{\phi_{2}}, m_{H_{3}}\right) C_{0}\left(q^{2} ; W, G^{ \pm}, H_{3}^{ \pm}\right) \\
& \left.-\left(c_{\phi_{1} V V} c_{\phi_{2} V V}+c_{\phi_{1} H_{3} V} c_{\phi_{2} H_{3} V}\right)\left(2 q^{2}-m_{\phi_{1}}^{2}-m_{\phi_{2}}^{2}\right) B_{0}\left(0 ; m_{W}, m_{G^{ \pm}}\right)\right],
\end{aligned}
$$

where we have introduced 


$$
\begin{aligned}
C_{0}\left(q^{2} ; A, B\right) & \equiv \frac{1}{m_{A}^{2}-m_{B}^{2}}\left[B_{0}\left(q^{2} ; m_{A}, m_{A}\right)-B_{0}\left(q^{2} ; m_{B}, m_{B}\right)\right], \\
C_{0}\left(q^{2} ; A, B, C\right) & \equiv \frac{1}{m_{A}^{2}-m_{B}^{2}}\left[B_{0}\left(q^{2} ; m_{A}, m_{C}\right)-B_{0}\left(q^{2} ; m_{B}, m_{C}\right)\right], \\
f\left(q^{2} ; m_{A}, m_{B}, m_{C}\right) & \equiv\left(q^{2}-m_{C}^{2}\right)^{2}-\left(m_{C}^{2}-m_{A}^{2}\right)\left(m_{C}^{2}-m_{B}^{2}\right) .
\end{aligned}
$$

The function $B_{0}$ is the Passarino-Veltman's scalar two-point function [39] defined in Sec. C 1 . We see that for $\phi_{1}=$ $\phi_{2}(\equiv \phi),\left.\Pi_{\phi_{1} \phi_{2}}\right|_{\text {G.D. }}$ vanishes at $q^{2}=m_{\phi}^{2}$ as it is expected by the Nielsen identity, so that the counterterms for the mass parameters $\left(\delta m_{h}^{2}\right.$ and $\left.\delta m_{H_{1}}^{2}\right)$ are gauge independent. For $\phi_{1} \neq \phi_{2},\left.\Pi_{\phi_{1} \phi_{2}}\right|_{\text {G.D. }}$ does not vanish either at $q^{2}=m_{\phi_{1}}^{2}$ or $q^{2}=m_{\phi_{2}}^{2}$, and thus the gauge dependence shows up in $\delta \alpha$.

In order to remove such gauge dependence, one can add pinch-term contributions to the above two-point functions. Pinch terms are the "propagatorlike" part of vertex and box-diagram corrections to a 2-to-2 fermion scattering process (i.e., $f \bar{f}^{\prime} \rightarrow f \bar{f}^{\prime}$ with $f$ and $f^{\prime}$ being SM fermions), and can be extracted by cancelling internal fermion propagators using a contracted loop momentum in the numerator. Here, the extracted pinch terms do not depend on the choice of the external fermions. Since the pinch terms also depend on the gauge choice, they can be expressed in a way similar to Eq. (47) as

$$
\Pi_{\phi_{1} \phi_{2}}^{\mathrm{PT}}\left(q^{2}\right)=\left.\Pi_{\phi_{1} \phi_{2}}^{\mathrm{PT}}\left(q^{2}\right)\right|_{\xi=1}+\left.\Pi_{\phi_{1} \phi_{2}}^{\mathrm{PT}}\left(q^{2}\right)\right|_{\mathrm{G} . \mathrm{D} .},
$$

where

$$
\begin{aligned}
\left.\Pi_{\phi_{1} \phi_{2}}^{\mathrm{PT}}\left(q^{2}\right)\right|_{\xi=1}= & -\frac{g^{2}}{16 \pi^{2}}\left(q^{2}-\frac{m_{\phi_{1}}^{2}}{2}-\frac{m_{\phi_{2}}^{2}}{2}\right) \\
& \times\left[c_{\phi_{1} V V} c_{\phi_{2} V V} B_{0}\left(q^{2} ; m_{W}, m_{W}\right)+c_{\phi_{1} H_{3} V} c_{\phi_{2} H_{3} V} B_{0}\left(q^{2} ; m_{H_{3}}, m_{W}\right)\right] .
\end{aligned}
$$

One can verify that the second term of Eq. (50) satisfies the property $\left.\Pi_{\phi_{1} \phi_{2}}^{\mathrm{PT}}\left(q^{2}\right)\right|_{\text {G.D. }}=-\left.\Pi_{\phi_{1} \phi_{2}}\left(q^{2}\right)\right|_{\text {G.D. }}$. Therefore, the twopoint functions with the pinch terms $\bar{\Pi}_{\phi_{1} \phi_{2}} \equiv \Pi_{\phi_{1} \phi_{2}}+\Pi_{\phi_{1} \phi_{2}}^{\mathrm{PT}}$ are gauge independent, and one should consider the gaugeindependent counterterm $\delta \bar{\alpha}$ defined by

$$
\delta \bar{\alpha}=\frac{1}{2\left(m_{H_{1}}^{2}-m_{h}^{2}\right)} \operatorname{Re}\left[\bar{\Pi}_{H_{1} h}\left(m_{H_{1}}^{2}\right)+\bar{\Pi}_{H_{1} h}\left(m_{h}^{2}\right)\right],
$$

instead of $\delta \alpha$, as we will do in the following discussions.

\section{B. CP-odd part}

Next, we discuss the gauge dependence of the two-point functions for the CP-odd scalar bosons $A_{1}-A_{2}$ $\left(A_{1,2} \in\left\{G^{0}, H_{3}^{0}\right\}\right)$. Similar to Eq. (48), we have

$$
\Pi_{A_{1} A_{2}}\left(q^{2}\right)=\left.\Pi_{A_{1} A_{2}}\left(q^{2}\right)\right|_{\xi=1}+\left.\Pi_{A_{1} A_{2}}\left(q^{2}\right)\right|_{\text {G.D. }} .
$$

The gauge-dependent part is expressed as

$$
\begin{aligned}
\left.\Pi_{A_{1} A_{2}}\left(q^{2}\right)\right|_{\text {G.D. }}= & \left(1-\xi_{W}\right) \frac{g^{2}}{32 \pi^{2}}\left\{\zeta_{A_{1}} \zeta_{A_{2}} f\left(q^{2} ; m_{A_{1}}, m_{A_{2}}, m_{H_{5}}\right) C_{0}\left(q^{2} ; W, G^{ \pm}, H_{5}^{ \pm}\right)\right. \\
& -\zeta_{A_{1}} \zeta_{A_{2}}\left(q^{2}-\frac{m_{A_{1}}^{2}}{2}-\frac{m_{A_{2}}^{2}}{2}\right) B_{0}\left(0 ; m_{W}, m_{G^{ \pm}}\right) \\
& \left.+\delta_{A_{1} H_{3}^{0}} \delta_{A_{2} H_{3}^{0}}\left(q^{2}-m_{H_{3}}^{2}\right)\left[\left(q^{2}-m_{H_{3}}^{2}\right) C_{0}\left(q^{2} ; W, G^{ \pm}, H_{3}^{ \pm}\right)-B_{0}\left(0 ; m_{W}, m_{G^{ \pm}}\right)\right]\right\} \\
& +\left(1-\xi_{Z}\right) \frac{g_{Z}^{2}}{64 \pi^{2}} \sum_{\varphi=h, H_{1}, H_{5}^{0}} c_{\varphi A_{1} Z} c_{\varphi A_{2} Z} \\
& \times\left[f\left(q^{2} ; m_{A_{1}}, m_{A_{2}}, m_{\varphi}\right) C_{0}\left(q^{2} ; Z, G^{0}, \varphi\right)-\left(q^{2}-\frac{m_{A_{1}}^{2}}{2}-\frac{m_{A_{2}}^{2}}{2}\right) B_{0}\left(0 ; m_{Z}, m_{G^{0}}\right)\right],
\end{aligned}
$$


where $\left(m_{A_{i}}, \zeta_{A_{i}}, c_{\varphi A_{1} Z}\right)=\left(m_{H_{3}},-s_{\beta},-c_{\varphi H_{3} Z}\right)$ for $A_{i}=H_{3}^{0}$ and $\left(0, c_{\beta}, c_{\varphi Z Z}\right)$ for $A_{i}=G^{0}$, and $c_{\varphi H_{3} Z}$ and $c_{\varphi Z Z}$ are given in Eqs. (B2) and (B5). Again, if $A_{1} \neq A_{2},\left.\Pi_{A_{1} A_{2}}\right|_{\text {G.D. }}$ does not vanish at either $q^{2}=m_{A_{1}}^{2}$ or $m_{A_{2}}^{2}$, and the counterterm $\delta \beta$ determined by Eq. (45) remains gauge dependent.

Analogous to the CP-even case, we can add the pinch terms extracted from a $f \bar{f}^{\prime} \rightarrow f \bar{f}^{\prime}$ process,

$$
\Pi_{A_{1} A_{2}}^{\mathrm{PT}}\left(q^{2}\right)=\left.\Pi_{A_{1} A_{2}}^{\mathrm{PT}}\left(q^{2}\right)\right|_{\xi=1}+\left.\Pi_{A_{1} A_{2}}^{\mathrm{PT}}\left(q^{2}\right)\right|_{\text {G.D. }},
$$

where the gauge-independent part

$$
\begin{aligned}
\left.\Pi_{A_{1} A_{2}}^{\mathrm{PT}}\left(q^{2}\right)\right|_{\xi=1}= & -\delta_{A_{1} H_{3}^{0}} \delta_{A_{2} H_{3}^{0}} \frac{g^{2}}{16 \pi^{2}}\left(q^{2}-m_{H_{3}}^{2}\right) B_{0}\left(q^{2} ; m_{W}, m_{H_{3}}\right) \\
& -\frac{g_{Z}^{2}}{32 \pi^{2}}\left(q^{2}-\frac{m_{A_{1}}^{2}}{2}-\frac{m_{A_{2}}^{2}}{2}\right) \sum_{\phi=h, H_{1}} c_{\phi A_{1} V} c_{\phi A_{2} V} B_{0}\left(q^{2} ; m_{Z}, m_{\phi}\right),
\end{aligned}
$$

while the gauge-dependent part

$$
\begin{aligned}
\left.\Pi_{A_{1} A_{2}}^{\mathrm{PT}}\left(q^{2}\right)\right|_{\text {G.D. }}= & -\delta_{A_{1} H_{3}^{0}} \delta_{A_{2} H_{3}^{0}}\left(1-\xi_{W}\right) \frac{g^{2}}{32 \pi^{2}}\left(q^{2}-m_{H_{3}}^{2}\right) \\
& \times\left[\left(q^{2}-m_{H_{3}}^{2}\right) C_{0}\left(q^{2} ; W, G^{ \pm}, H_{3}^{ \pm}\right)-B_{0}\left(0 ; m_{W}, m_{G^{ \pm}}\right)\right] \\
& -\left(1-\xi_{Z}\right) \frac{g_{Z}^{2}}{64 \pi^{2}}\left\{\sum _ { \phi = h , H } c _ { \phi A _ { 1 } Z } c _ { \phi A _ { 2 } Z } \left[f\left(q^{2} ; m_{A_{1}}, m_{A_{2}}, m_{\phi}\right) C_{0}\left(q^{2} ; Z, G^{0}, \phi\right)\right.\right. \\
& \left.-\left(q^{2}-\frac{m_{A_{1}}^{2}}{2}-\frac{m_{A_{2}}^{2}}{2}\right) B_{0}\left(0 ; m_{Z}, m_{G^{0}}\right)\right] \\
& \left.-\frac{5}{3} \zeta_{A_{1}} \zeta_{A_{2}}\left(q^{2}-\frac{m_{A_{1}}^{2}}{2}-\frac{m_{A_{2}}^{2}}{2}\right) B_{0}\left(0 ; m_{Z}, m_{G^{0}}\right)\right\} .
\end{aligned}
$$

Analogous to Eq. (52), one should consider the pinch-termincluded counterterm $\delta \bar{\beta}$ defined by

$$
\delta \bar{\beta}=-\frac{1}{2 m_{H_{3}}^{2}} \operatorname{Re}\left[\bar{\Pi}_{G^{0} H_{3}^{0}}(0)+\bar{\Pi}_{G^{0} H_{3}^{0}}\left(m_{H_{3}}^{2}\right)\right]
$$

where $\bar{\Pi}_{A_{1} A_{2}} \equiv \Pi_{A_{1} A_{2}}+\Pi_{A_{1} A_{2}}^{\mathrm{PT}}$. It is intriguing to note that $\left.\Pi_{A_{1} A_{2}}^{\mathrm{PT}}\right|_{\text {G.D. }} \neq-\left.\Pi_{A_{1} A_{2}}\right|_{\text {G.D. }}$ in this case; that is, $\delta \bar{\beta}$ still has explicit gauge dependence. In fact, the 5-plet Higgs boson loop contributions to $\left.\Pi_{A_{1} A_{2}}\right|_{\text {G.D. }}$, the terms proportional to $\zeta_{A_{1}} \zeta_{A_{2}}$ and $c_{H_{5}^{0} A_{1} Z} c_{H_{5}^{0} A_{2} Z}$ in Eq. (54), are not cancelled by the pinch terms because of the fermio-phobic nature of the 5-plet Higgs bosons. Therefore, even after the pinch terms are included, gauge dependence still remains in the twopoint functions for the CP-odd scalar bosons. This does not happen, for example, in the THDMs, where the gauge dependence in the two-point functions for the CP-odd Higgs bosons does cancel by adding the pinch terms, as shown in Refs. [15,34].

To see the $\xi$ dependence, we introduce

$$
\Delta \hat{\beta} \equiv \frac{\left.\hat{\beta}\right|_{\xi}-\left.\hat{\beta}\right|_{\xi=1}}{\left.\hat{\beta}\right|_{\xi=1}}, \quad \hat{\beta}=\beta+\left.\delta \bar{\beta}\right|_{\text {fin }}
$$

where $\left.\delta \bar{\beta}\right|_{\text {fin }}$ is the finite part of $\delta \bar{\beta}$. In Fig. 1, we show how the renormalized mixing angle $\hat{\beta}$ depends on the choice of the gauge parameter $\xi\left(=\xi_{W}=\xi_{Z}\right)$. We see that the gauge dependence of $\hat{\beta}$ becomes larger for larger values of $\xi$ and/ or $v_{\Delta}$, but it is at most about $1 \%$ or smaller when $\xi \leq 10^{3}$. Therefore, in the numerical evaluation for the renormalized Higgs boson vertices, the actual effect from the gauge dependence in $\delta \bar{\beta}$ is negligibly small. Moreover, the modifications cause by varying $\alpha$ between $0^{\circ}$ and $-20^{\circ}$, a range of phenomenological interest, are much minor. In the following discussion, we will use $\delta \bar{\beta}$ instead of $\delta \beta$.

Before closing this section, we would like to remark that the gauge dependence of the two-point function for the CP-odd Higgs bosons is cancelled if we add up all the contributions to the $f \bar{f}^{\prime} \rightarrow f \bar{f}^{\prime}$ scattering amplitude from the $H_{3}^{0}-H_{3}^{0}, H_{3}^{0}-G^{0}$ and $G^{0}-G^{0}$ mediators:

$$
\begin{aligned}
\mathcal{M}= & -\frac{m_{f} m_{f^{\prime}}}{v^{2}}\left(\bar{f} \gamma_{5} f\right)\left(\bar{f}^{\prime} \gamma_{5} f^{\prime}\right) \\
& \times\left[\left(\frac{\cot \beta}{q^{2}-m_{H_{3}}^{2}}\right)^{2} \bar{\Pi}_{H_{3}^{0} H_{3}^{0}}\left(q^{2}\right)\right. \\
& \left.+\frac{2 \cot \beta}{q^{2}\left(q^{2}-m_{H_{3}}^{2}\right)} \bar{\Pi}_{H_{3}^{0} G^{0}}\left(q^{2}\right)+\frac{1}{q^{4}} \bar{\Pi}_{G^{0} G^{0}}\left(q^{2}\right)\right] .
\end{aligned}
$$



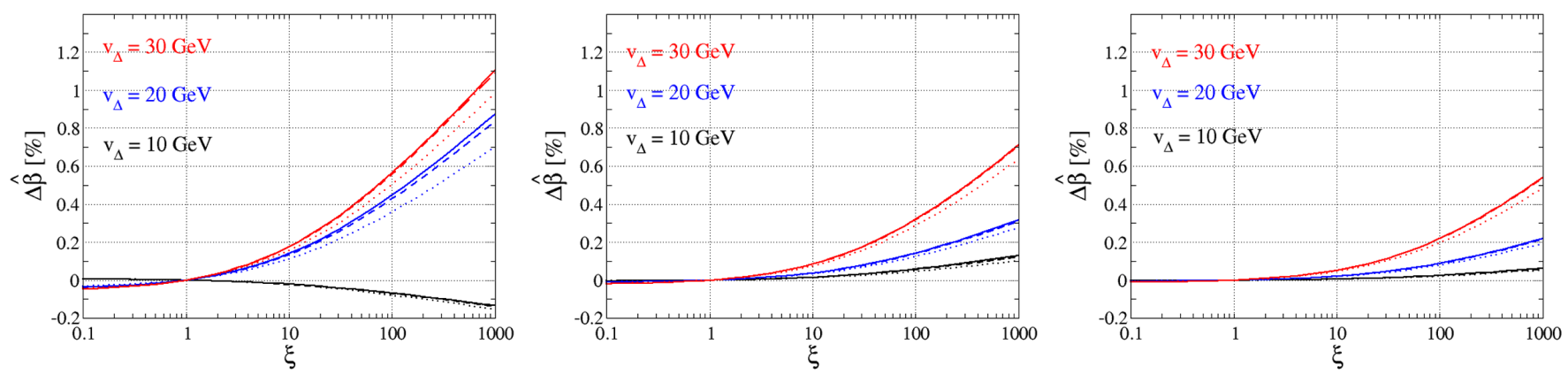

FIG. 1. Gauge dependence of the renormalized mixing angle $\hat{\beta}$ in the case of $\mu_{1}=-100 \mathrm{GeV}$ and $\mu_{2}=0$ with $\xi_{W}=\xi_{Z}=\xi$, where $\Delta \hat{\beta}$ is defined in Eq. (59). The left, middle and right plots show the case with $m_{H_{5}}=m_{H_{3}}=m_{H_{1}}=300,500$, and $700 \mathrm{GeV}$, respectively. For each plot, the black, blue and red curves show, respectively, the cases of $v_{\Delta}=10,20$, and $30 \mathrm{GeV}$, while the solid, dashed and dotted curves denote respectively the case with $\alpha=0^{\circ},-10^{\circ}$, and $-20^{\circ}$.

One can explicitly verify that the gauge dependence in the above expression is exactly cancelled among the three terms in the square brackets.

\section{RENORMALIZED HIGGS VERTICES}

In this section, we compute renormalized $h V^{\mu} V^{\nu}$ $(V=W, Z), h f \bar{f}$ and $h h h$ vertices based on the on-shell scheme discussed in Sec. III. We note that the on-shell conditions are insufficient to fix all the counterterms appearing in the renormalized $h h h$ vertex. Therefore, we have to introduce an additional condition, the $\overline{\mathrm{MS}}$ scheme, to be discussed in Sec. V C. All the analytic expressions for the 1PI diagram contributions (variables labeled with the superscript "1PI") to one-, two-, and three-point functions are given in Appendix C.

Hereafter, we use the shorthand notation for the trigonometric function as $s_{\theta}=\sin \theta, c_{\theta}=\cos \theta$, and $t_{\theta}=\tan \theta$.

\section{A. Renormalized $h V V$ vertex}

The renormalized $h V^{\mu} V^{\nu}$ vertices can be generally decomposed as

$$
\hat{\Gamma}_{h V V}^{\mu \nu}\left(p_{1}^{2}, p_{2}^{2}, q^{2}\right)=\hat{\Gamma}_{h V V}^{1} g^{\mu \nu}+\hat{\Gamma}_{h V V}^{2} \frac{p_{1}^{\nu} p_{2}^{\mu}}{m_{V}^{2}}+i \hat{\Gamma}_{h V V}^{3} \epsilon^{\mu \nu \rho \sigma} \frac{p_{1 \rho} p_{2 \sigma}}{m_{V}^{2}},
$$

where $p_{1,2}^{\mu}$ and $q^{\mu}\left(=p_{1}^{\mu}+p_{2}^{\mu}\right)$ are the incoming momenta of the gauge bosons and the outgoing momentum of $h$, respectively. Each of the renormalized form factors $\hat{\Gamma}_{h V V}^{i}$ can be further decomposed into four parts as

$$
\hat{\Gamma}_{h V V}^{i}\left(p_{1}^{2}, p_{2}^{2}, q^{2}\right)=\Gamma_{h V V}^{i, \text { tree }}+\delta \Gamma_{h V V}^{i}+\Gamma_{h V V}^{i, 1 \mathrm{PI}}\left(p_{1}^{2}, p_{2}^{2}, q^{2}\right)+T_{h V V}^{i}, \quad(i=1,2,3),
$$

where $\Gamma_{h V V}^{i, \text { tree }}, \delta \Gamma_{h V V}^{i}, \Gamma_{h V V}^{i, 1 \mathrm{PI}}$, and $T_{h V V}^{i}$ denote the contributions from tree-level diagrams, counterterms, 1PI diagrams, and tadpoles, respectively. We note that the tadpole part $\Pi_{A B}^{\mathrm{Tad}}$ in the counterterms is here grouped into $T_{h V V}^{i}$. According to Eq. (26),

$$
T_{h V V}^{i}=\delta \Gamma_{h V V}^{i}(\text { tadpole part })+\Gamma_{h V V}^{i, T a d} .
$$

Each term in Eq. (62) is given as follows:

$$
\begin{aligned}
\Gamma_{h V V}^{1, \text { tree }}= & \frac{2 m_{V}^{2}}{v} c_{h V V}, \\
\delta \Gamma_{h V V}^{1}= & \frac{2 m_{V}^{2}}{v}\left[c_{h V V}\left(\frac{\delta m_{V}^{2}}{m_{V}^{2}}-\frac{\delta v}{v}+\delta Z_{V}+\frac{1}{2} \delta Z_{h}\right)\right. \\
& \left.-c_{h H_{3} V} \delta \bar{\beta}+C_{\nu}^{V} \frac{\delta \nu}{v}+\frac{c_{H_{1} V V}}{2} \delta Z_{H_{1} h}+\frac{c_{H_{5} V V}}{2} \delta Z_{H_{5} h}\right], \\
T_{h V V}^{1, \mathrm{Tad}}= & \frac{2 m_{V}^{2}}{v^{2} m_{H_{3}}^{2}} c_{h H_{3} V}\left(T_{h}^{1 \mathrm{PI}} c_{h H_{3} V}+T_{H_{1}}^{1 \mathrm{PI}} c_{H_{1} H_{3} V}+T_{H_{5}^{0}}^{1 \mathrm{PI}} c_{H_{5} H_{3} Z}\right),
\end{aligned}
$$

where $C_{\nu}^{W}=\sqrt{2} s_{\beta} c_{h H_{3} V}$ and $C_{\nu}^{Z}=\sqrt{2} c_{\beta} c_{h V V}$. For $i=2,3$, we have $\Gamma_{h V V}^{i, \text { tree }}=\delta \Gamma_{h V V}^{i}=T_{h V V}^{i}=0$. As mentioned in the previous section, we adopt the pinched counterterm $\delta \bar{\beta}$ defined in Eq. (58) instead of $\delta \beta$ given in Eq. (45). 


\section{B. Renormalized $h f \bar{f}$ vertex}

The renormalized $h f \bar{f}$ vertices can be expressed in terms of eight form factors as follows,

$$
\begin{aligned}
\hat{\Gamma}_{h f f}\left(p_{1}^{2}, p_{2}^{2}, q^{2}\right)= & \hat{\Gamma}_{h f f}^{S}+\gamma_{5} \hat{\Gamma}_{h f f}^{P}+\not p_{1} \hat{\Gamma}_{h f f}^{V_{1}}+\not p_{2} \hat{\Gamma}_{h f f}^{V_{2}} \\
& +\not \not_{1} \gamma_{5} \hat{\Gamma}_{h f f}^{A_{1}}+\not \not_{2} \gamma_{5} \hat{\Gamma}_{h f f}^{A_{2}}+\not \not_{1} \not \not_{2} \hat{\Gamma}_{h f f}^{T}+\not \not_{1} \not \not_{2} \gamma_{5} \hat{\Gamma}_{h f f}^{P T},
\end{aligned}
$$

where $p_{1,2}^{\mu}$ and $q^{\mu}\left(=p_{1}^{\mu}+p_{2}^{\mu}\right)$ are the incoming momenta of the fermions and the outgoing momentum of $h$, respectively. Analogous to the renormalized $h V^{\mu} V^{\nu}$ vertices, each of the renormalized form factors is further decomposed into the following four parts:

$$
\hat{\Gamma}_{h f f}^{i}\left(p_{1}^{2}, p_{2}^{2}, q^{2}\right)=\Gamma_{h f f}^{i, \text { tree }}+\delta \Gamma_{h f f}^{i}+\Gamma_{h f f}^{i, 1 \mathrm{PI}}+T_{h f f}^{i},
$$

where $i=S, P, V_{1}, V_{2}, A_{1}, A_{2}, T, P T$. We note that the tadpole term cannot be inserted to the tree-level $h f \bar{f}$ diagram; that is, $\Gamma_{h f f}^{\mathrm{Tad}}=0$. Hence, the tadpole contribution $T_{h f f}^{i}$ is obtained only from the corresponding counterterm. Each term of Eq. (66), except for the 1PI part, is given as follows,

$$
\begin{aligned}
\Gamma_{h f f}^{S, \text { tree }} & =-\frac{m_{f}}{v} c_{h f f}, \\
\delta \Gamma_{h f f}^{S} & =-\frac{m_{f}}{v} c_{h f f}\left(\frac{\delta m_{f}}{m_{f}}+\delta Z_{V}^{f}-\frac{\delta v}{v}-\cot \beta \delta \bar{\beta}+\sqrt{2} c_{\beta} \frac{\delta \nu}{v}+\frac{\delta Z_{h}}{2}+\frac{c_{H f f}}{2 c_{h f f}} \delta Z_{H_{1} h}\right), \\
T_{h f f}^{S} & =-\frac{m_{f}}{v} c_{h f f} \frac{\cot \beta}{m_{H_{3}}^{2} v}\left(c_{h H_{3} V} T_{h}^{\mathrm{lPI}}+c_{H_{1} H_{3} V} T_{H_{1}^{0}}^{\mathrm{PI}}+\frac{2 \sqrt{3}}{3} s_{\beta} T_{H_{5}^{0}}^{\mathrm{PI}}\right),
\end{aligned}
$$

with $\Gamma_{h f f}^{i, \text { tree }}=\delta \Gamma_{h f f}^{i}=T_{h f f}^{i}=0 \quad$ for $i \neq S$. As for the renormalized $h V^{\mu} V^{\nu}$ vertex, we also use the pinched counterterm $\delta \bar{\beta}$ in the contribution to the $h f \bar{f}$ vertex.

\section{Renormalized hhh vertex}

Finally, we compute the renormalized $h h h$ vertex which is trivial in the Lorentz structure as it is a scalar vertex. Analogous to the $h V^{\mu} V^{\nu}$ and $h f \bar{f}$ vertices, the renormalized $h h h$ vertex can be expressed as

$$
\hat{\Gamma}_{h h h}\left(p_{1}^{2}, p_{2}^{2}, q^{2}\right)=\Gamma_{h h h}^{\mathrm{tree}}+\delta \Gamma_{h h h}+\Gamma_{h h h}^{1 \mathrm{PI}}+T_{h h h},
$$

where $p_{1,2}^{\mu}$ and $q^{\mu}\left(=p_{1}^{\mu}+p_{2}^{\mu}\right)$ are the incoming and outgoing momenta for the Higgs boson, respectively. Each of the contributions is given as follows:

$$
\begin{aligned}
\Gamma_{h h h}^{\mathrm{tree}}= & 3 ! \lambda_{h h h}=-\frac{3 m_{h}^{2}}{v}\left(\frac{c_{\alpha}^{3}}{s_{\beta}}-\frac{2 \sqrt{6}}{3} \frac{s_{\alpha}^{3}}{c_{\beta}}\right)+\mu_{1} s_{\alpha}^{2} t_{\beta}\left(3 \sqrt{2} c_{\alpha}+2 \sqrt{3} s_{\alpha} t_{\beta}\right)-2 \sqrt{3} \mu_{2} s_{\alpha}^{3}, \\
\delta \Gamma_{h h h}= & 3 !\left[\delta \lambda_{h h h}+\frac{3}{2} \lambda_{h h h} \delta Z_{h}+\frac{1}{2} \lambda_{H_{1} h h}\left(\delta Z_{H_{1} h}+2 \delta \bar{\alpha}\right)\right] \\
T_{h h h}= & 3 !\left\{C\left(m_{h}^{2}\right) \frac{m_{h}^{2}}{v}\left[2 \sum_{\phi=h, H_{1}}\left(1+2 \delta_{\phi h}\right) \frac{\lambda_{\phi h h} T_{\phi}^{1 \mathrm{PI}}}{m_{h}^{2} m_{\phi}^{2}}+\sum_{\varphi=h, H_{1}, H_{5}^{0}} c_{\varphi W W} \frac{T_{\varphi}^{1 \mathrm{PI}}}{v m_{\varphi}^{2}}\right]\right. \\
& +\sum_{\phi=h, H_{1}}\left[c_{h H_{3} V} \frac{s_{2 \alpha}}{s_{2 \beta}} \lambda_{\phi H h} \frac{T_{\phi}^{1 \mathrm{PI}}}{v m_{\phi}^{2}}+\left(1+3 \delta_{\phi h}\right) \lambda_{\phi h h h} \frac{T_{\phi}^{1 \mathrm{PI}}}{m_{\phi}^{2}}\right] \\
& \left.-C(\delta \beta) \sum_{\varphi=h, H_{1}, H_{5}^{0}} c_{\varphi H_{3} Z} \frac{m_{\varphi}^{2}-m_{H_{3}}^{2}}{m_{H_{3}}^{2}} \frac{T_{\varphi}^{1 \mathrm{PI}}}{m_{\varphi}^{2}}+\frac{3}{\sqrt{6}} C(\delta \nu) \frac{T_{H_{5}^{0}}^{1 \mathrm{PI}}}{m_{H_{5}}^{2}}\right\},
\end{aligned}
$$

where $\lambda_{\phi_{i} \phi_{j} \phi_{k}}$ and $\lambda_{\phi_{i} \phi_{j} \phi_{k} \phi_{l}}$ are defined in Eq. (C9), and 


$$
\begin{aligned}
C\left(\delta m_{h}^{2}\right) & =-\frac{1}{3 s_{2 \beta}}\left(3 c_{\alpha}^{3} c_{\beta}-2 \sqrt{6} s_{\alpha}^{3} s_{\beta}\right), \\
C(\delta \beta) & =\left(\frac{c_{\alpha}^{3}}{2 t_{\beta} s_{\beta}}+\frac{\sqrt{6}}{3} s_{\alpha}^{3} \frac{t_{\beta}}{c_{\beta}}\right) \frac{m_{h}^{2}}{v^{2}}+s_{\alpha}^{2} \frac{3 \sqrt{2} c_{\alpha}+4 \sqrt{3} s_{\alpha} t_{\beta}}{6 c_{\beta}^{2}} \frac{\mu_{1}}{v}, \\
C(\delta \nu) & =\left[\frac{2 \sqrt{3}}{9} s_{\alpha}^{3}\left(3-\frac{2}{c_{\beta}^{2}}\right)-\frac{\sqrt{2} c_{\alpha}^{3}}{2 t_{\beta}}\right] \frac{m_{h}^{2}}{v^{2}}-\frac{2 c_{\alpha} s_{\alpha}^{2} s_{\beta}}{9 c_{\beta}^{2}}\left(3+2 \sqrt{6} t_{\alpha} t_{\beta}\right) \frac{\mu_{1}}{v} .
\end{aligned}
$$

The counterterm $\delta \lambda_{h h h}$ is expressed as

$$
\begin{aligned}
\delta \lambda_{h h h}= & C\left(\delta m_{h}^{2}\right) \frac{m_{h}^{2}}{v}\left(\frac{\delta m_{h}^{2}}{m_{h}^{2}}-\frac{\delta v}{v}\right)+\left(\frac{c_{h H_{3} V}}{2} \frac{s_{2 \alpha}}{s_{2 \beta}} \frac{m_{H_{1}}^{2}-m_{h}^{2}}{v}-\lambda_{H_{1} h h}\right) \delta \bar{\alpha} \\
& +C(\delta \beta) v \delta \bar{\beta}+C(\delta \nu) \delta \nu+\frac{s_{\alpha}^{2} t_{\beta}}{6}\left(3 \sqrt{2} c_{\alpha}+2 \sqrt{3} s_{\alpha} t_{\beta}\right) \delta \mu_{1}-\frac{s_{\alpha}^{3}}{\sqrt{3}} \delta \mu_{2} .
\end{aligned}
$$

Notice here that $\delta \alpha$ and $\delta \beta$ are correctly replaced by the corresponding pinched counterterms $\delta \bar{\alpha}$ and $\delta \bar{\beta}$.

In Eq. (71), the counterterms $\delta \mu_{1}$ and $\delta \mu_{2}$ show up and cannot be individually determined by applying the on-shell scheme, as alluded to in the beginning of the section. A similar situation also happens in THDMs [8] and the HSM [6]. In this paper, we apply the $\overline{\mathrm{MS}}$ scheme to fix the combination of $\delta \mu_{1}$ and $\delta \mu_{2}$, where these counterterms are determined so as to cancel only the UV divergent part $\Delta_{\text {div }}$ of $\delta \Gamma_{h h h}$ (without the $\delta \mu_{1}$ and $\delta \mu_{2}$ terms), $\Gamma_{h h h}^{1 \mathrm{PI}}$ and $T_{h h h}$. Here, $\Delta_{\text {div }} \equiv 1 / \epsilon+\ln 4 \pi-\gamma_{E}$ with $\epsilon$ being defined in Appendix $\mathrm{C} 1$ and $\gamma_{E}$ being the Euler-Mascheroni constant. The similar method has also been applied to fix the counterterm for the $h h h$ vertex in THDMs $[8,11]$ and that in the HSM [6]. We note that because of the usage of the $\overline{\mathrm{MS}}$ scheme, the scale dependence $\mu$, defined in Appendix C 1, appears in the renormalized $h h h$ vertex.

\section{Renormalized Higgs boson couplings}

We can now calculate the renormalized Higgs boson vertices (i.e., $h V V, h f \bar{f}$ and $h h h$ vertices) from the discussions in the previous subsections. We here define the renormalized scale factors $\hat{\kappa}_{X}$ for the Higgs boson couplings, which are convenient to discuss the deviation in the couplings from the SM predictions, as follows,

$$
\begin{gathered}
\hat{\kappa}_{V}\left(p^{2}\right) \equiv \frac{\hat{\Gamma}_{h V V}^{1}\left(m_{V}^{2}, p^{2}, m_{h}^{2}\right)_{\mathrm{GM}}}{\hat{\Gamma}_{h V V}^{1}\left(m_{V}^{2}, p^{2}, m_{h}^{2}\right)_{\mathrm{SM}}}, \\
\hat{\kappa}_{t}\left(p^{2}\right) \equiv \frac{\hat{\Gamma}_{h t t}^{S}\left(m_{t}^{2}, p^{2}, m_{h}^{2}\right)_{\mathrm{GM}}}{\hat{\Gamma}_{h t t}^{S}\left(m_{t}^{2}, p^{2}, m_{h}^{2}\right)_{\mathrm{SM}}}, \\
\hat{\kappa}_{h}\left(p^{2}\right) \equiv \frac{\hat{\Gamma}_{h h h}\left(m_{h}^{2}, m_{h}^{2}, p^{2}\right)_{\mathrm{GM}}}{\hat{\Gamma}_{h h h}\left(m_{h}^{2}, m_{h}^{2}, p^{2}\right)_{\mathrm{SM}}},
\end{gathered}
$$

where $p^{2}$ denotes the squared momentum for the off-shell particle, namely, $V^{*}, t^{*}$, and $h^{*}$ for the $h V V$, $h t \bar{t}$, and $h h h$ couplings, respectively. For the $h b \bar{b}$ and $h \tau^{+} \tau^{-}$couplings, we define their renormalized scale factors without the momentum dependence since the on-shell decays $h \rightarrow$ $b \bar{b}$ and $h \rightarrow \tau^{+} \tau^{-}$are allowed:

$$
\hat{\kappa}_{f} \equiv \frac{\hat{\Gamma}_{h f f}^{S}\left(m_{f}^{2}, m_{f}^{2}, m_{h}^{2}\right)_{\mathrm{GM}}}{\hat{\Gamma}_{h f f}^{S}\left(m_{f}^{2}, m_{f}^{2}, m_{h}^{2}\right)_{\mathrm{SM}}} \quad \text { for } f=b, \tau .
$$

For the numerical evaluation of these scale factors, we use the following SM input parameters [21]:

$$
\begin{aligned}
& G_{F}=1.166379 \times 10^{-5} \mathrm{GeV}^{-2}, \quad \alpha_{\mathrm{EM}}^{-1}=137.035999, \quad \Delta \alpha_{\mathrm{EM}}=0.06635, \\
& m_{Z}=91.1876 \mathrm{GeV}, \quad m_{h}=125 \mathrm{GeV}, \quad m_{t}=173.21 \mathrm{GeV}, \\
& m_{b}=4.66 \mathrm{GeV}, \quad m_{c}=1.275 \mathrm{GeV}, \quad m_{\tau}=1.77684 \mathrm{GeV},
\end{aligned}
$$

where all the other quarks and leptons are assumed to be massless.

In order to understand the typical behaviors of the renormalized scale factors, we show $\hat{\kappa}_{W}, \hat{\kappa}_{Z}, \hat{\kappa}_{t}$ and $\hat{\kappa}_{h}$ as functions of the mixing angle $\alpha$ in Fig. 2. In these plots, the squared momentum is fixed to be $(250 \mathrm{GeV})^{2}$ for $\hat{\kappa}_{W, Z}$ and
$(500 \mathrm{GeV})^{2}$ for $\hat{\kappa}_{t, h}$. Moreover, the masses of the extra Higgs bosons are taken to be $400 \mathrm{GeV}$ in common, and the triplet VEV $v_{\Delta}$ is set to be $10 \mathrm{GeV}$ (black), $20 \mathrm{GeV}$ (blue), and $30 \mathrm{GeV}$ (red). The other parameters $\mu_{1}$ and $\mu_{2}$ are scanned in ranges that are taken as large as possible so as to maximize the allowed region of $\alpha$ for a fixed value of $v_{\Delta}$. In 

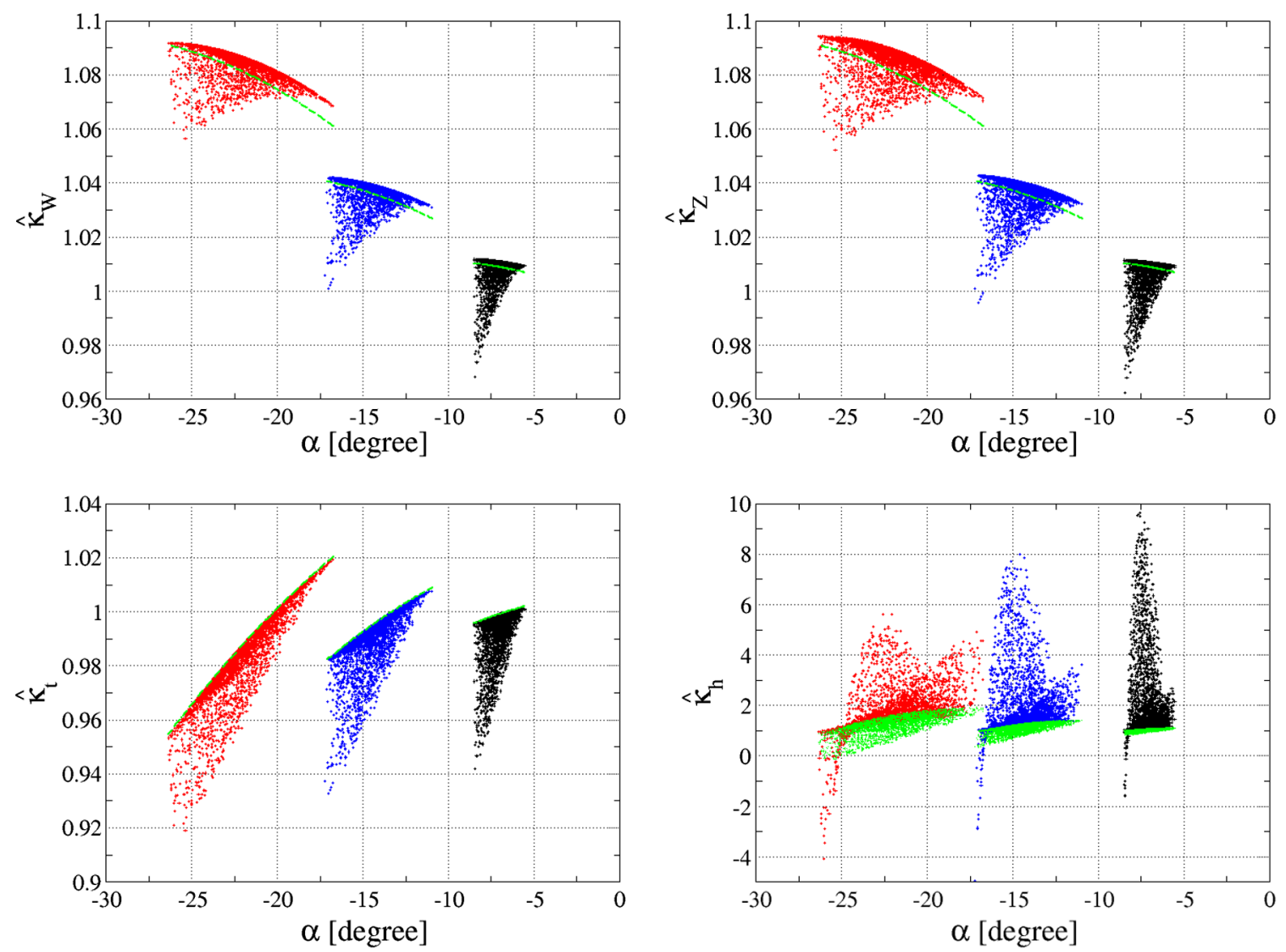

FIG. 2. Renormalized scale factors $\hat{\kappa}_{W}$ (upper left), $\hat{\kappa}_{Z}$ (upper right), $\hat{\kappa}_{t}$ (lower left) and $\hat{\kappa}_{h}$ (lower left) as functions of $\alpha$ for $m_{H_{5}}=m_{H_{3}}=m_{H_{1}}=400 \mathrm{GeV}$, where the squared momentum is taken to be $(250 \mathrm{GeV})^{2}$ for $\hat{\kappa}_{W}$ and $\hat{\kappa}_{Z}$ and $(500 \mathrm{GeV})^{2}$ for $\hat{\kappa}_{t}$ and $\hat{\kappa}_{h}$. In these plots, $v_{\Delta}$ is fixed to be $10 \mathrm{GeV}$ (black), $20 \mathrm{GeV}$ (blue) and $30 \mathrm{GeV}$ (red), while $\mu_{1}$ and $\mu_{2}$ are scanned. The tree-level predictions are also shown by the dashed green curves (for $\hat{\kappa}_{W}, \hat{\kappa}_{Z}$ and $\hat{\kappa}_{t}$ ) and green dots (for $\hat{\kappa}_{h}$ ). We only show the points allowed by the constraints of perturbative unitarity and vacuum stability, to be described in Sec. VI A.

addition, we impose the constraints from perturbative unitarity and vacuum stability, to be described in Sec. VI A in the parameter scan. The dots and curves shown in this figure satisfy both of these constraints.

We see that larger values of $\hat{\kappa}_{Z}$ and $\hat{\kappa}_{W}$ are obtained for larger values of $v_{\Delta}$ and $|\alpha|$. This behavior can roughly be explained by the dominant tree-level prediction shown by the green dashed curves, determined solely by $\alpha$ and $\beta$ (or equivalently $v_{\Delta}$ ) in Eq. (B2). That is, the term proportional to $2 \sqrt{6} / 3$ in $c_{h V V}$ becomes important for larger $v_{\Delta}$ and $|\alpha|$. We also see that the quantum effect typically reduces the values of $\hat{\kappa}_{Z}$ and $\hat{\kappa}_{W}$ by a few percent with respect to the tree-level predictions, where the most important source of quantum corrections comes from the counterterm for the $h$ wave function $\delta Z_{h}$ in Eq. (64). In fact, the 5-plet Higgs boson loop contributions to $\delta Z_{h}$ provide a term proportional to $\lambda_{H_{5} H_{5} h}^{2}$ (see Eq. (C15) and notice $\delta Z_{h}=$ $\left.-\Pi_{h h}^{\prime}\left(m_{h}^{2}\right)\right)$ defined in Eq. (C10), which can be significant depending on $\mu_{1}$ and $\mu_{2}$, and it determines the typical size of quantum corrections.

Similar to $\hat{\kappa}_{W}$ and $\hat{\kappa}_{Z}$, the behavior of $\hat{\kappa}_{t}$ is roughly determined by the tree-level prediction, i.e., $c_{h f f}$ given in
Eq. (B8). In fact, it is seen that $\hat{\kappa}_{t}$ becomes small when we take larger values of $v_{\Delta}$ and $|\alpha|$. In addition, the quantum correction reduces $\hat{\kappa}_{t}$, mainly because of the effect of $\delta Z_{h}$. We note that the predictions for $\hat{\kappa}_{b}$ and $\hat{\kappa}_{\tau}$ are almost the same as that of $\hat{\kappa}_{t}$.

For $\hat{\kappa}_{h}$, there are several features different from $\hat{\kappa}_{W}, \hat{\kappa}_{Z}$ and $\hat{\kappa}_{t}$. First of all, the tree-level prediction, shown by the green dots, is not a single-valued curve, but spreads over a region on the plane. This is because $\hat{\kappa}_{h}$ depends not only on $\alpha$ and $v_{\Delta}$ but also on $\mu_{1}$ and $\mu_{2}$ as seen in Eq. (69). Secondly, $\hat{\kappa}_{h}$ can receive a large quantum correction at several $100 \%$ level with respect to the tree-level prediction. This large correction can be ascribed to the $\lambda_{H_{5} H_{5} h}^{3}$ dependence in the 1PI diagram contribution, see Eq. (C34), when the value of $|\alpha|$ is not close to its maximum for a given value of $v_{\Delta}$.

While the predicted scale factors presented here are only for some special cases, we will show their behaviors in more generic cases in Sec. VII.

Finally, we discuss the momentum dependence of $\hat{\kappa}_{W}$, $\hat{\kappa}_{Z}, \hat{\kappa}_{t}$ and $\hat{\kappa}_{h}$. We provide six benchmark points (BP1BP6), all of which are allowed by both the constraints of 
TABLE I. Six benchmark points allowed by the perturbative unitarity and the vacuum stability. The masses of the extra Higgs bosons are taken to be $m_{H_{5}}=m_{H_{3}}=m_{H_{1}}=400 \mathrm{GeV}$. All the other input parameters are shown in the first four columns. The numbers given in the latter four columns show the output of the renormalized scale factors at $\sqrt{p^{2}}=250(500) \mathrm{GeV}$ for $\hat{\kappa}_{W, Z}\left(\hat{\kappa}_{t, h}\right)$.

\begin{tabular}{lcccccccc}
\hline \hline & $\alpha[$ degree $]$ & $v_{\Delta}[\mathrm{GeV}]$ & $\mu_{1}[\mathrm{GeV}]$ & $\mu_{2}[\mathrm{GeV}]$ & $\hat{\kappa}_{W}$ & $\hat{\kappa}_{Z}$ & $\hat{\kappa}_{t}$ & $\hat{\kappa}_{h}$ \\
\hline BP1 & -7.0 & 10 & -100.3 & 112.3 & 1.01 & 1.01 & 1.00 & 1.08 \\
BP2 & -8.0 & 10 & 7.1 & 2789.5 & 0.99 & 0.98 & 0.96 & 4.71 \\
BP3 & -15.1 & 20 & -180.0 & 171.0 & 1.04 & 1.04 & 0.99 & 1.34 \\
BP4 & -16.1 & 20 & 18.7 & 1338.3 & 1.02 & 1.01 & 0.95 & 4.00 \\
BP5 & -22.4 & 30 & -325.2 & -53.3 & 1.09 & 1.09 & 0.98 & 1.39 \\
BP6 & -24.9 & 30 & 10.0 & 755.0 & 1.07 & 1.06 & 0.93 & 0.87 \\
\hline \hline
\end{tabular}

perturbative unitarity and vacuum stability. In Table I, we show the input parameters of BP1-BP6 and the output values of the renormalized scale factors at $\sqrt{p^{2}}=$ $250(500) \mathrm{GeV}$ for $\hat{\kappa}_{W, Z}\left(\hat{\kappa}_{t, h}\right)$. BP1, BP3, and BP5 (BP2, $\mathrm{BP} 4$, and BP6) are chosen such that the predictions of oneloop corrected scale factors are close to (far from) the treelevel predictions for $v_{\Delta}=10,20$ and $30 \mathrm{GeV}$, respectively.

In Fig. 3, the momentum dependence of $\hat{\kappa}_{V}, \hat{\kappa}_{t}$ and $\hat{\kappa}_{h}$ are shown for the six benchmark points. For $\hat{\kappa}_{V}$, both $\hat{\kappa}_{W}$ and $\hat{\kappa}_{Z}$ monotonically increase with $\sqrt{p^{2}}$, where the increasing rates for $\mathrm{BP} 2, \mathrm{BP} 4$, and $\mathrm{BP} 6$ are more significant as compared to those for $\mathrm{BP} 1, \mathrm{BP} 3$, and $\mathrm{BP} 5$. We also observe that the increasing rates becomes slightly higher at $\sqrt{p^{2}} \gtrsim 800 \mathrm{GeV}$ because of the threshold effects of the extra Higgs bosons. In addition, the difference between $\hat{\kappa}_{W}$ and $\hat{\kappa}_{Z}$ is getting larger as $\sqrt{p^{2}}$ increases. On the other hand, the momentum dependence of $\hat{\kappa}_{t}$ is quite mild with compared to that of $\hat{\kappa}_{V}$, where the results for BP3 and BP6, having larger values of $|\alpha|$ and $v_{\Delta}$, show more significant momentum dependence as compared to the other benchmark points. For $\hat{\kappa}_{h}$, we see the sharp dip (peak) for BP1, $\mathrm{BP} 3$, and BP5 (BP2, BP4, and BP6) at around $\sqrt{p^{2}}=$ $800 \mathrm{GeV}$ due to the threshold effects of the extra Higgs boson loops, where the appearance of the dip/peak can be explained by the destructive/constructive interference of the
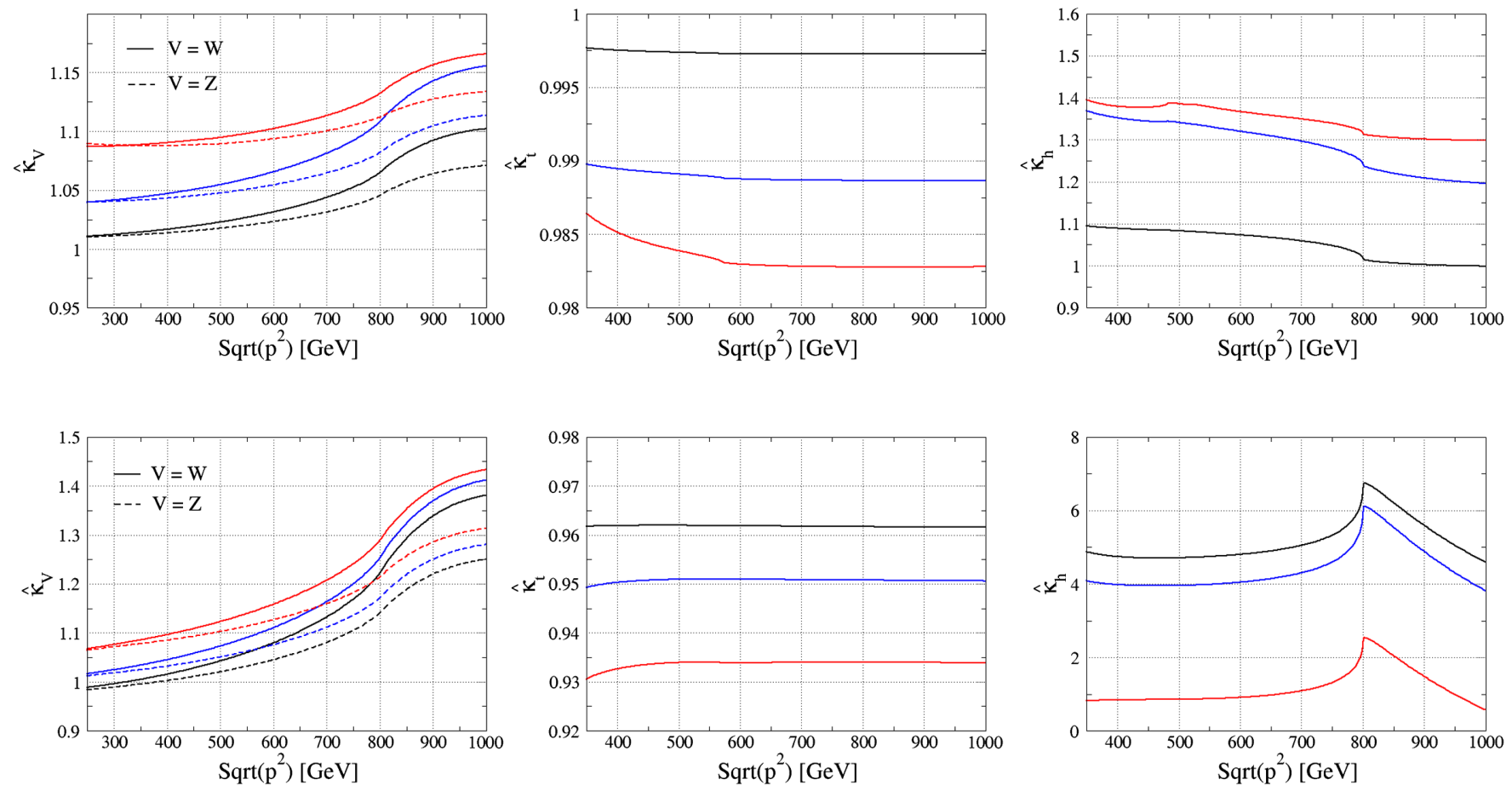

FIG. 3. Renormalized scale factors $\hat{\kappa}_{W, Z}$ (left), $\hat{\kappa}_{t}$ (middle) and $\hat{\kappa}_{h}$ (right) as functions of $\sqrt{p^{2}}$ for $m_{H_{5}}=m_{H_{3}}=m_{H_{1}}=400 \mathrm{GeV}$. The upper panels show the cases of BP1 (black), BP3 (blue), and BP5 (red), while the lower panels show the cases of BP2 (black), BP4 (blue), and BP6 (red). 
$\lambda_{H_{5} H_{5} h}^{3}$ and $\lambda_{H_{5} H_{5} h} \lambda_{H_{5} H_{5} h h}$ terms in Eq. (C34), i.e., the signs of these two contributions flip between (BP1, BP3, BP5) and (BP2, BP4, BP6).

\section{CONSTRAINTS ON PARAMETER SPACE}

In this section, we discuss both theoretical and experimental constraints that we impose on the model parameters. A search of viable exotic Higgs boson mass spectra based upon similar constraints and prospects for detecting the doubly charged Higgs boson at the 14-TeV LHC and a $100-\mathrm{TeV}$ future $p p$ collider had been studied in Ref. [40].

\section{A. Theoretical bounds}

Two theoretical constraints are taken into account to constrain the dimensionless quartic couplings of the scalar potential at tree level: the stability of the electroweak vacuum and the unitarity of the perturbation theory. These constraints on the quartic couplings can be translated into bounds on the physical parameters such as the masses and mixing angles of the Higgs bosons through the relations given in Appendix A.

The vacuum stability requires the scalar potential to be bounded from below and leads to the following constraints for the quartic couplings [31]:

$$
\begin{aligned}
& \lambda_{1}>0, \\
& \lambda_{2}> \begin{cases}-\frac{1}{3} \lambda_{3} & \text { for } \lambda_{3} \geq 0, \\
-\lambda_{3} \quad \text { for } \lambda_{3}<0, & \text { for } \lambda_{5} \leq 0 \text { and } \lambda_{3} \geq 0,\end{cases} \\
& \lambda_{4}>\left\{\begin{array}{ll}
-\frac{1}{2} \lambda_{5}-2 \sqrt{\lambda_{1}\left(\frac{1}{3} \lambda_{3}+\lambda_{2}\right)} & \text { for } \lambda_{5} \leq 0 \text { and } \lambda_{3}<0, \\
-\omega_{+}(\zeta) \lambda_{5}-2 \sqrt{\lambda_{1}\left(\zeta \lambda_{3}+\lambda_{2}\right)} & \text { for } \lambda_{5}>0,
\end{array}-\omega_{-}(\zeta) \lambda_{5}-2 \sqrt{\lambda_{1}\left(\zeta \lambda_{3}+\lambda_{2}\right)}\right.
\end{aligned}
$$

where

$$
\omega_{ \pm}(\zeta)=\frac{1}{6}(1-B) \pm \frac{\sqrt{2}}{3}\left[(1-B)\left(\frac{1}{2}+B\right)\right]^{1 / 2},
$$

with $B$ randomly varying between 0 and 1 .

The bound from perturbative unitarity is obtained by requiring that the $s$-wave amplitude matrix, $a_{0}$, for elastic $2 \rightarrow 2$ scalar boson scatterings does not become too large to violate $S$-matrix unitarity. One can set the criteria for this requirement as that the magnitudes of all the eigenvalues of $a_{0}$ do not exceed $1 / 2$. In the high-energy limit, the matrix elements of $a_{0}$ are expressed by the scalar quartic couplings because only the diagrams involving scalar contact interactions are relevant. In this setup, one can obtain the following conditions $[31,41]$ :

$$
\begin{aligned}
& \left|6 \lambda_{1}+7 \lambda_{3}+11 \lambda_{2} \pm \sqrt{\left(6 \lambda_{1}-7 \lambda_{3}-11 \lambda_{2}\right)^{2}+36 \lambda_{4}^{2}}\right|<4 \pi \\
& \left|2 \lambda_{1}-\lambda_{3}+2 \lambda_{2} \pm \sqrt{\left(2 \lambda_{1}+\lambda_{3}-2 \lambda_{2}\right)^{2}+\lambda_{5}^{2}}\right|<4 \pi \\
& \left|\lambda_{4}+\lambda_{5}\right|<2 \pi, \quad\left|2 \lambda_{3}+\lambda_{2}\right|<\pi, \\
& \left|2 \lambda_{2}+\lambda_{3}\right|<2 \pi, \quad\left|4 \lambda_{4}+\lambda_{5}\right|<8 \pi, \quad\left|2 \lambda_{4}-\lambda_{5}\right|<4 \pi .
\end{aligned}
$$

We note that by combining the vacuum stability condition, the first two inequalities of (77) can be simply replaced by

$$
\begin{gathered}
\left|6 \lambda_{1}+7 \lambda_{3}+11 \lambda_{2}\right|+\sqrt{\left(6 \lambda_{1}-7 \lambda_{3}-11 \lambda_{2}\right)^{2}+36 \lambda_{4}^{2}}<4 \pi, \\
\left|2 \lambda_{1}-\lambda_{3}+2 \lambda_{2}\right|+\sqrt{\left(2 \lambda_{1}+\lambda_{3}-2 \lambda_{2}\right)^{2}+\lambda_{5}^{2}}<4 \pi .
\end{gathered}
$$

\section{B. Experimental bounds}

Next, we discuss the experimental constraints from the electroweak oblique $S$ parameter, the signal strengths for the $125 \mathrm{GeV}$ Higgs boson, and the direct searches for extra Higgs bosons. We note that the oblique $T$ parameter is used as one of the inputs (see the discussion in Sec. III A), so that it cannot be applied to the constrain the GM model. We require that predictions of these observables in the model be within the $95 \%$ confidence level (CL) region. In the following, we explain how these constraints from experimental data are imposed in our analysis, in order.

The current electroweak data fit gives [21]

$$
S=0.07 \pm 0.08,
$$

by fixing $U=0$.

The Higgs signal strengths have been measured from 20 channels with different combinations of production and decay channels in Ref. [42]. Among these measurements, we do not include the signal strengths for the $Z h$ and $t h$ 

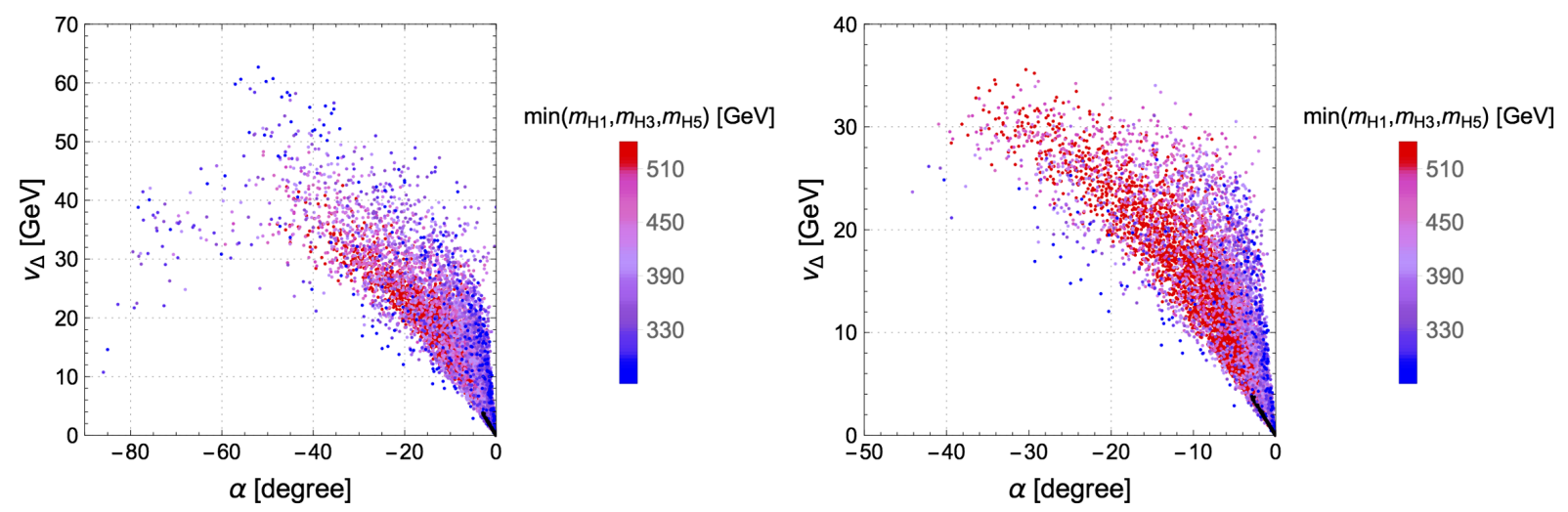

FIG. 4. Points allowed by Set-A constraints (left plot) and Set-B constraints (right plot). The color of the dots shows the value of $\min \left(m_{H_{1}}, m_{H_{3}}, m_{H_{5}}\right)$. The black dots are obtained by changing the scan range of $m_{\Delta}$ to $(1,2.5) \mathrm{TeV}$ while keeping the scan ranges of all the other parameters as in Eq. (80).

productions with the $h \rightarrow W W^{*}$ decay in our study because the SM predictions for these two channels are excluded by the current data at $95 \% \mathrm{CL}$. It should be noted that channels with $h$ decaying into a pair of photons provide effective constraints on the masses of extra Higgs bosons as their dependences appear in the charged Higgs boson $\left(H_{3}^{ \pm}, H_{5}^{ \pm}\right.$ and $H_{5}^{ \pm \pm}$) loop contributions to the $h \rightarrow \gamma \gamma$ decay. In contrast, all the other channels depend on only two parameters: $\alpha$ and $\beta$.

The constraint from the direct search for the doubly charged Higgs boson $H_{5}^{ \pm \pm}$is imposed. A bound on the production cross section of $H_{5}^{ \pm \pm}$via the vector boson fusion mechanism times the branching fraction of the $H_{5}^{ \pm \pm} \rightarrow W^{ \pm} W^{ \pm}$decay has been set by the CMS Collaboration at the collision energy of $8 \mathrm{TeV}$ and the integrated luminosity of $19.4 \mathrm{fb}^{-1}$ [43]. This constraint can

$$
\begin{array}{lrrr}
-0.628 \leq \lambda_{2} \leq 1.57, & -1.57 \leq \lambda_{3} \leq 1.88, & -2.09 \leq \lambda_{4} \leq 2.09, & -8.38 \leq \lambda_{5} \leq 8.38 \\
-650 \leq \mu_{1} \leq 0 \mathrm{GeV}, & -400 \leq \mu_{2} \leq 50 \mathrm{GeV}, & 180 \leq m_{\Delta} \leq 450 \mathrm{GeV} .
\end{array}
$$
independent free parameters in the GM model as shown
The ranges of $\lambda_{2,3}$ are determined by the constraints from perturbative unitarity and vacuum stability, while those of $\lambda_{4,5}$ are determined by the bounds from the perturbative unitarity only [31]. The parameter scan is performed under two sets of constraints: Set-A takes into account the constraints of vacuum stability, perturbative unitarity and the $S$ parameter, and Set-B further considers the Higgs signal strengths and direct search of $H_{5}^{ \pm \pm}$, all at 95\% CL.

In Fig. 4, points allowed by Set-A constraints (left plot) and Set-B constraints (right plot) are shown in the $\alpha-v_{\Delta}$ plane. The color of the dots indicates the value of $\min \left(m_{H_{1}}, m_{H_{3}}, m_{H_{5}}\right)$, the minimum of the exotic Higgs boson masses. It is seen that Set-B constraints exclude regions with larger values of $|\alpha|$ and $v_{\Delta}$ in comparison with be translated into the bound on the mass of the 5-plet Higgs bosons $m_{H_{5}}$ and the triplet VEV $v_{\Delta}$.

\section{Allowed parameter space}

We are now ready to present the allowed parameter space by imposing the constraints discussed in the previous subsections.

After fixing $v$ and $m_{h}$, there are, in total, seven in Eq. (11), assuming the custodial symmetry at tree level. Instead of using the parameters given in Eq. (11), we choose four dimensionless quartic couplings $\lambda_{2-5}$ and three dimensionful parameters $\mu_{1,2}$ and $m_{\Delta}$ in the Higgs potential as our inputs, with which all the other parameters are determined. We then perform a scan of the parameters in the following ranges:

using only Set-A constraints. These two parameters are constrained to be $-40^{\circ} \lesssim \alpha \lesssim 0^{\circ}$ and $v_{\Delta} \lesssim 35 \mathrm{GeV}$ under Set-B constraints. We can also see from the right plot that for a fixed smaller value of $|\alpha|$ (e.g., $|\alpha| \lesssim 10^{\circ}$ ), the maximally allowed value of $\min \left(m_{H_{1}}, m_{H_{3}}, m_{H_{5}}\right)$ becomes smaller as $v_{\Delta}$ increases. On the other hand, larger values of $v_{\Delta}$ and $\min \left(m_{H_{1}}, m_{H_{3}}, m_{H_{5}}\right)$ can be found for larger $|\alpha|$. In order to numerically check the decoupling behavior, we also add the black dots which are obtained by scanning $1 \leq m_{\Delta} \leq 2.5 \mathrm{TeV}$ while keeping the scan ranges of all the other parameters as in Eq. (80). These black dots also correspond to $\min \left(m_{H_{1}}, m_{H_{3}}, m_{H_{5}}\right)$ from 1.4 to $3.5 \mathrm{TeV}$. As expected, the decoupling limit $m_{\Delta} \gg v$ resides in the region where both $|\alpha|$ and $v_{\Delta}$ approach zero. 

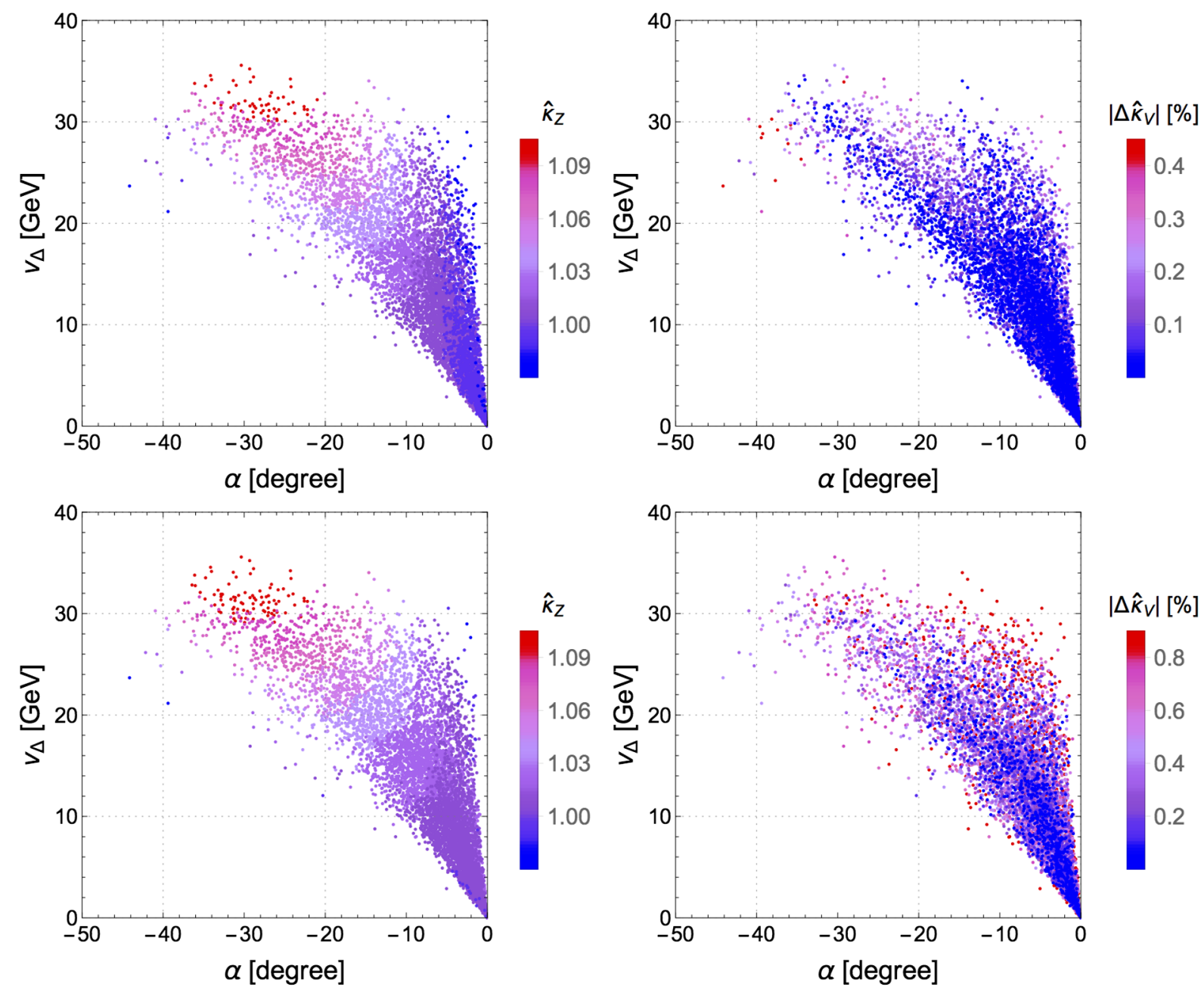

FIG. 5. Scatter plots of $\hat{\kappa}_{Z}$ (left) and $\left|\Delta \hat{\kappa}_{V}\right|$ (right) for $\sqrt{p^{2}}=250 \mathrm{GeV}$ (upper) and $\sqrt{p^{2}}=500 \mathrm{GeV}$ (lower) in the $\alpha-v_{\Delta}$ plane.

\section{NUMERICAL RESULTS FOR RENORMALIZED HIGGS BOSON COUPLINGS}

In this section, we numerically show the deviations in the one-loop corrected $h V V, h f \bar{f}$ and $h h h$ couplings from the corresponding SM predictions under the scan defined in Eq. (80). These deviations are expressed in terms of the renormalized scale factors $\hat{\kappa}_{X}$ defined in Eqs. (72) and (73). In the following analysis, we restrict ourselves to the parameter region allowed by Set-B constraints defined in Sec. VI C.

First, we show the behavior of the scale factors for the Higgs couplings with weak gauge bosons. Moreover, we define

$$
\Delta \hat{\kappa}_{V}\left(p^{2}\right) \equiv \hat{\kappa}_{Z}\left(p^{2}\right)-\hat{\kappa}_{W}\left(p^{2}\right) .
$$

Figure 5 shows the scatter plots of $\hat{\kappa}_{Z}$ (left plots) and $\left|\Delta \hat{\kappa}_{V}\right|$ (right plots) for $\sqrt{p^{2}}=250 \mathrm{GeV}$ (upper plots) and $500 \mathrm{GeV}$ (lower plots) in the $\alpha-v_{\Delta}$ plane. It is clear from the left plots that larger values of $\hat{\kappa}_{Z}$ are obtained in the region with larger values of $v_{\Delta}$ and $|\alpha|$. The result for $\hat{\kappa}_{Z}$ does not change much as we change from $\sqrt{p^{2}}=250$ to $500 \mathrm{GeV}$, in agreement with the special case in Fig. 3. We note that within our parameter scan ranges, $\hat{\kappa}_{Z}$ varies from $0.88(0.93)$ to $1.12(1.13)$ for $\sqrt{p^{2}}=250$ (500) GeV. On the other hand, from the right plots we see that maximal $\left|\Delta \hat{\kappa}_{V}\right|$ is typically around $0.2 \%$ for $\sqrt{p^{2}}=250 \mathrm{GeV}$, while the maximum becomes around $0.8 \%$ for $\sqrt{p^{2}}=500 \mathrm{GeV}$. It is also seen that $\left|\Delta \hat{\kappa}_{V}\right|$ does not depend on $v_{\Delta}$ and $\alpha$ so much. We note that $\Delta \hat{\kappa}_{V}$ can be either positive or negative, and it falls in the range of $-0.7 \%$ to $0.4 \%$ for $\sqrt{p^{2}}=250 \mathrm{GeV}$ and $0.0 \%$ to $1.45 \%$ for $\sqrt{p^{2}}=500 \mathrm{GeV}$.

In Fig. 6, we show the scatter plots of $\hat{\kappa}_{b}$ (left) and $\hat{\kappa}_{t}$ with $\sqrt{p^{2}}=500 \mathrm{GeV}$ (right) in the $\alpha-v_{\Delta}$ plane. As shown, the behaviors of $\hat{\kappa}_{b}$ and $\hat{\kappa}_{t}$ are almost the same as each other. The result for $\hat{\kappa}_{\tau}$ is also very similar to that of $\hat{\kappa}_{b}$. In contrast to the case of $\hat{\kappa}_{Z}$, the value of $\hat{\kappa}_{b}$ becomes smaller when $|\alpha|$ becomes larger.

Figure 7 is a scatter plot of $\hat{\kappa}_{h}$ for $\sqrt{p^{2}}=500 \mathrm{GeV}$. While the variations from the SM predictions in Figs. 5 and 6 are typically less than about $10 \%$, the magnitude of 

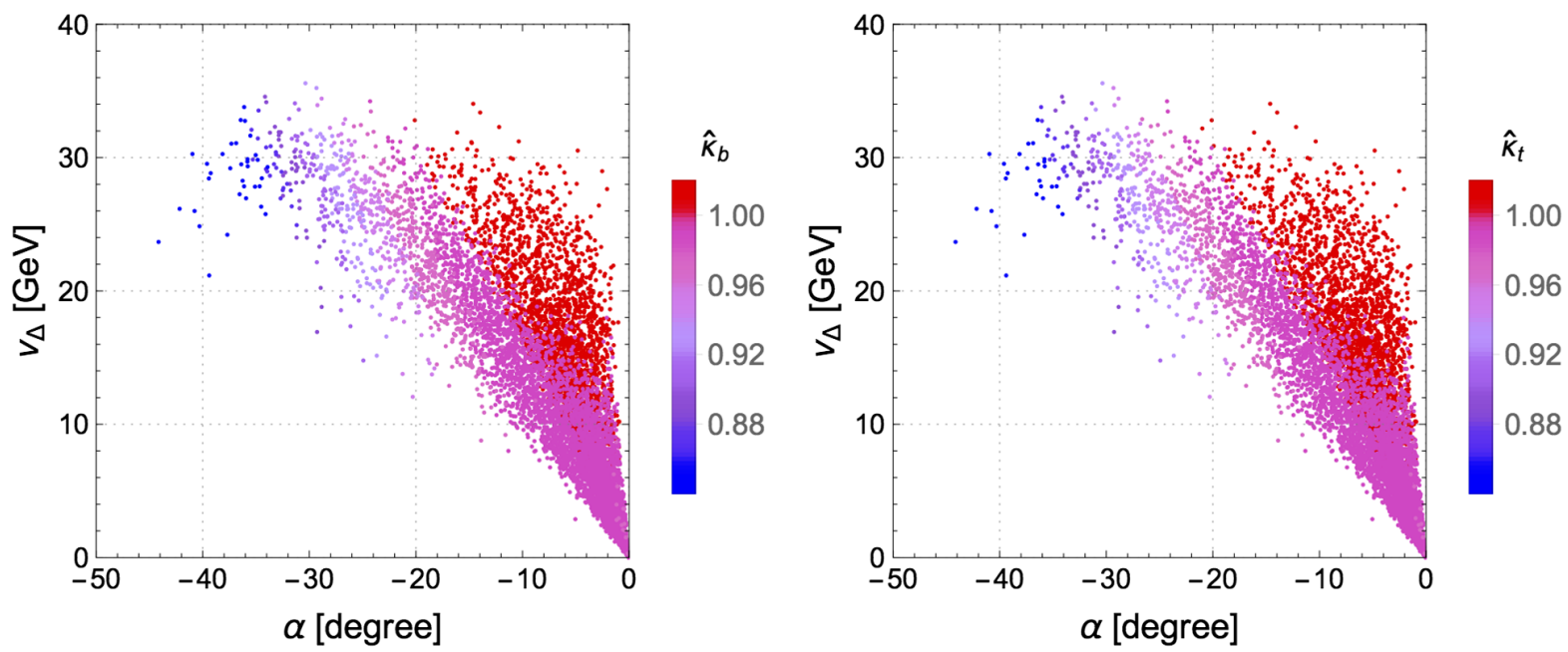

FIG. 6. Scatter plots of $\hat{\kappa}_{b}$ (left) and $\hat{\kappa}_{t}$ (right) in the $\alpha-v_{\Delta}$ plane. For $\hat{\kappa}_{t}$, we take $\sqrt{p^{2}}=500 \mathrm{GeV}$.

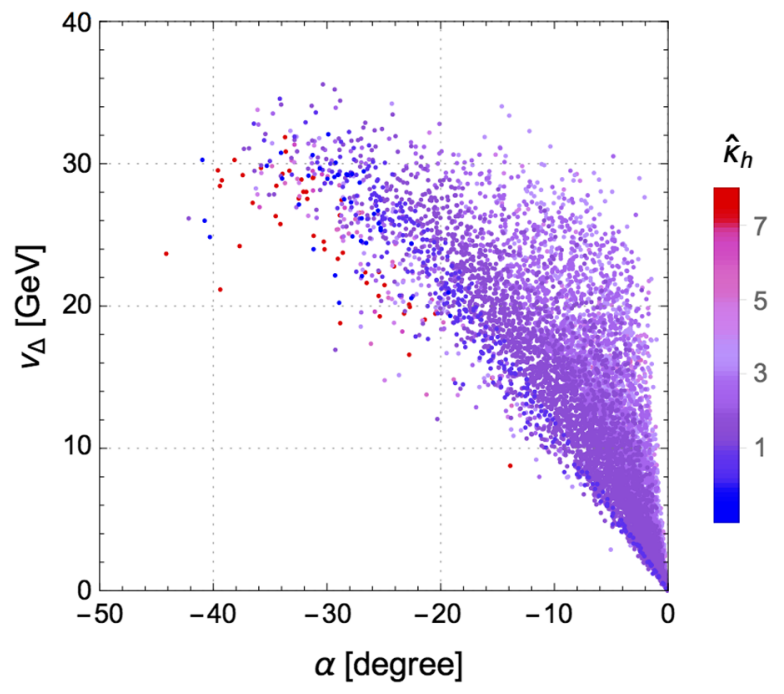

FIG. 7. Scatter plot of $\hat{\kappa}_{h}$ with $\sqrt{p^{2}}=500 \mathrm{GeV}$.

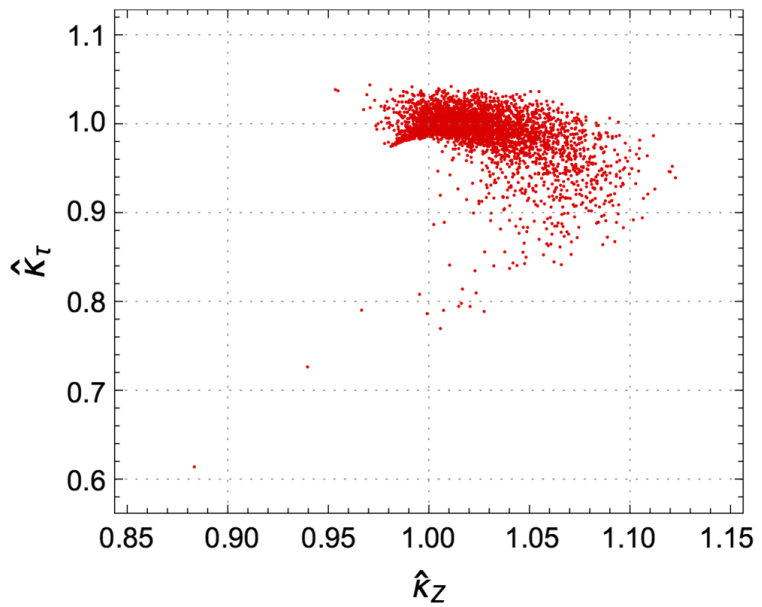

the deviation in the $h h h$ coupling, i.e., $\hat{\kappa}_{h}-1$, can be at a few $100 \%$ level. In addition, $\hat{\kappa}_{h}$ does not depend much on $\alpha$ and $v_{\Delta}$ as compared to $\hat{\kappa}_{Z}, \hat{\kappa}_{b}$ and $\hat{\kappa}_{t}$.

Finally, we show the correlation of the renormalized scale factors. Figure 8 shows the correlation between $\hat{\kappa}_{Z}$ and $\hat{\kappa}_{\tau}$, where the momentum $\sqrt{p^{2}}$ of $\hat{\kappa}_{Z}$ is set to be $250 \mathrm{GeV}$ and $500 \mathrm{GeV}$ in the left and right plots, respectively. We see that the distribution of the dots in the $\hat{\kappa}_{Z}-\hat{\kappa}_{\tau}$ plane for $\sqrt{p^{2}}=500 \mathrm{GeV}$ is almost the same as that for $\sqrt{p^{2}}=250 \mathrm{GeV}$, except for slight shrinking in the range of $\hat{\kappa}_{Z}$ in the former case. It is also seen that the range of possible $\kappa_{\tau}$ gets restricted when $\hat{\kappa}_{Z}$ becomes larger. At $\hat{\kappa}_{Z} \simeq 1.13, \hat{\kappa}_{\tau}$ is predicted to be about 0.95 .

Figure 9 shows the correlation between $\hat{\kappa}_{Z}$ and $\hat{\kappa}_{h}$, where the momentum $\sqrt{p^{2}}$ of $\hat{\kappa}_{Z}$ is set to be 250 and $500 \mathrm{GeV}$ in the left and right plots, respectively, while that of $\hat{\kappa}_{h}$ is fixed at $500 \mathrm{GeV}$ for both plots. Aside from some shifting in the dot distributions between the two plots, most of the

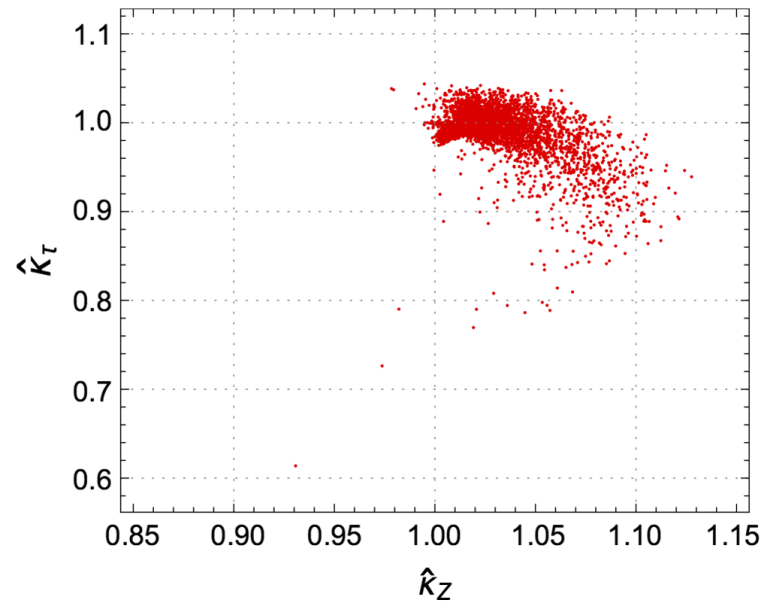

FIG. 8. Correlation between $\hat{\kappa}_{Z}$ and $\hat{\kappa}_{\tau}$, where $\sqrt{p^{2}}$ of $\hat{\kappa}_{Z}$ is taken to be 250 (left) and $500 \mathrm{GeV}$ (right). 

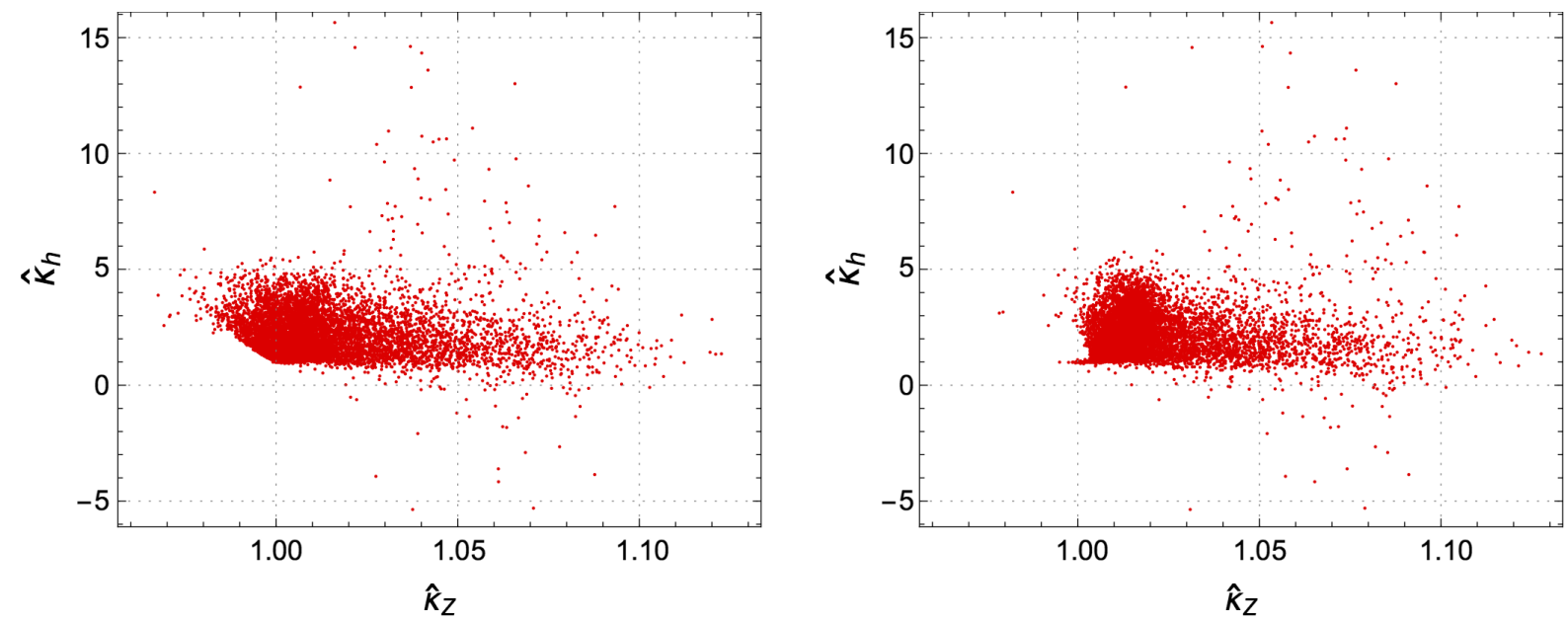

FIG. 9. Correlation between $\hat{\kappa}_{Z}$ and $\hat{\kappa}_{h}$, where $\sqrt{p^{2}}$ of $\hat{\kappa}_{Z}$ is taken to be 250 (left) and $500 \mathrm{GeV}$ (right), while $\sqrt{p^{2}}$ of $\hat{\kappa}_{h}$ is fixed at $500 \mathrm{GeV}$ for both plots.

predicted $\hat{\kappa}_{h}$ values are between 1 and 5. Again, the possible range of $\kappa_{h}$ is restricted when $\hat{\kappa}_{Z}$ becomes larger. In particular, $\hat{\kappa}_{h}$ is predicted to be about 1 when $\hat{\kappa}_{Z} \simeq 1.13$. We also notice some of the predicted $\hat{\kappa}_{h}$ values are less than 1 or even negative for $\hat{\kappa}_{Z} \gtrsim 1$.

\section{CONCLUSIONS}

In this work, we have calculated the one-loop renormalized vertices of the $125 \mathrm{GeV}$ Higgs boson $(h)$ with the weak gauge bosons $(h V V)$, fermions $(h f f)$, and itself $(h h h)$ in the GM model. We have chosen to work with the on-shell renormalization scheme and the $\overline{\mathrm{MS}}$ scheme, where the latter is only applied to the determination of the counterterms appearing in the renormalized $h h h$ coupling. Special care has been taken to check gauge dependence of the counterterms for the mixing parameters $\delta \alpha$ and $\delta \beta$. We have defined the gauge-independent counterterm $\delta \alpha$ by adding the pinchterm contributions to the mixing two-point functions for the CP-even Higgs bosons. For $\delta \beta$, we have clarified that its gauge dependence cannot be removed completely even if we add the pinch-term contributions to the mixing two-point functions for the CP-odd Higgs bosons, due to the gaugephilic and fermio-phobic nature of the 5-plet Higgs bosons. Such gauge dependence, however, is exactly cancelled in 2-to-2 fermion scattering processes.

We have numerically evaluated the renormalized Higgs boson couplings, subject to the theoretical bounds of perturbative unitarity and vacuum stability at tree level.
We have further imposed the constraints from experimental data: the oblique $S$ parameter, the Higgs signal strengths and the direct search for doubly charged Higgs boson. It has been found that the magnitudes of deviations in the oneloop-corrected $h V V$ and $h f \bar{f}$ couplings from the SM predictions can be up to about $10 \%$ level, where the signs of the deviations are typically positive and negative for the $h V V$ and $h f \bar{f}$ couplings, respectively. The one-loop corrected hhh coupling, on the other hand, can be significantly larger than the SM prediction by several hundred percent. Finally, we have studied and shown the correlations of renormalized scale factors for the Higgs boson couplings.

\section{ACKNOWLEDGMENTS}

This research of CWC was supported in part by the Ministry of Science and Technology of Taiwan under Grant No. MOST 104-2628-M-002-014-MY4.

\section{APPENDIX A: MASS EIGENSTATES OF THE SCALAR FIELDS}

In this Appendix, we give the mass eigenstates of the scalar fields in the GM model and their masses as derived from the potential in Eqs. (6) and (7).

The mass eigenstates of the scalar fields are related to the original fields given in Eq. (1) by the following transformations,

$$
\begin{aligned}
\left(\begin{array}{c}
\chi_{i} \\
\phi_{i}
\end{array}\right) & =\left(\begin{array}{cc}
c_{\beta_{\text {odd }}} & -s_{\beta_{\text {odd }}} \\
s_{\beta_{\text {odd }}} & c_{\beta_{\text {odd }}}
\end{array}\right)\left(\begin{array}{c}
G^{0} \\
H_{3}^{0}
\end{array}\right), \\
\left(\begin{array}{c}
\phi^{ \pm} \\
\xi^{ \pm} \\
\chi^{ \pm}
\end{array}\right) & =R_{H_{5}^{ \pm}} R_{\beta_{ \pm}} R_{\gamma}\left(\begin{array}{c}
G^{ \pm} \\
H_{3}^{ \pm} \\
H_{5}^{ \pm}
\end{array}\right), \quad\left(\begin{array}{c}
\xi_{r} \\
\phi_{r} \\
\chi_{r}
\end{array}\right)=R_{H_{5}^{0}} R_{\alpha}\left(\begin{array}{c}
H_{1} \\
h \\
H_{5}^{0}
\end{array}\right),
\end{aligned}
$$


where $G^{ \pm}$and $G^{0}$ are the NG bosons to become the longitudinal components of $W^{ \pm}$and $Z$ bosons, respectively. The rotation matrices in Eq. (A1)

$$
\begin{aligned}
R_{H_{5}^{ \pm}} & =\left(\begin{array}{ccc}
1 & 0 & 0 \\
0 & \frac{1}{\sqrt{2}} & -\frac{1}{\sqrt{2}} \\
0 & \frac{1}{\sqrt{2}} & \frac{1}{\sqrt{2}}
\end{array}\right), \quad R_{\beta_{ \pm}}=\left(\begin{array}{ccc}
s_{\beta_{1}^{ \pm}} & c_{\beta_{1}^{ \pm}} & 0 \\
c_{\beta_{1}^{ \pm}} & -s_{\beta_{1}^{ \pm}} & 0 \\
0 & 0 & 1
\end{array}\right)\left(\begin{array}{ccc}
c_{\beta_{2}^{ \pm}} & 0 & s_{\beta_{2}^{ \pm}} \\
0 & 1 & 0 \\
-s_{\beta_{2}^{ \pm}} & 0 & c_{\beta_{2}^{ \pm}}
\end{array}\right), \\
R_{\gamma} & =\left(\begin{array}{ccc}
1 & 0 & 0 \\
0 & c_{\gamma} & -s_{\gamma} \\
0 & s_{\gamma} & c_{\gamma}
\end{array}\right), \quad R_{H_{5}^{0}}=\left(\begin{array}{ccc}
\frac{1}{\sqrt{3}} & 0 & -\sqrt{\frac{2}{3}} \\
0 & 1 & 0 \\
\sqrt{\frac{2}{3}} & 0 & \frac{1}{\sqrt{3}}
\end{array}\right), \\
R_{\alpha} & =\left(\begin{array}{ccc}
1 & 0 & 0 \\
0 & c_{\alpha_{1}} & -s_{\alpha_{1}} \\
0 & s_{\alpha_{1}} & c_{\alpha_{1}}
\end{array}\right)\left(\begin{array}{ccc}
c_{\alpha_{2}} & 0 & -s_{\alpha_{2}} \\
0 & 1 & 0 \\
s_{\alpha_{2}} & 0 & c_{\alpha_{2}}
\end{array}\right)\left(\begin{array}{ccc}
c_{\alpha_{3}} & -s_{\alpha_{3}} & 0 \\
s_{\alpha_{3}} & c_{\alpha_{3}} & 0 \\
0 & 0 & 1
\end{array}\right),
\end{aligned}
$$

with the mixing angles satisfying

$$
\tan \beta_{\text {odd }}=\frac{v_{\phi}}{2 \sqrt{2} v_{\Delta}}, \quad \tan \beta_{1}^{ \pm}=\frac{v_{\phi}}{\sqrt{2}\left(2 v_{\Delta}+\nu\right)}, \quad \tan \beta_{2}^{ \pm}=\frac{\sqrt{2} \nu}{\sqrt{v_{\phi}^{2}+2\left(2 v_{\Delta}+\nu\right)^{2}}} .
$$

The other mixing angles $\gamma, \alpha_{1}, \alpha_{2}$, and $\alpha_{3}$ generally have very complicated forms. Nevertheless, an important thing is that in the $\nu \rightarrow 0$ limit $\gamma, \alpha_{1}$, and $\alpha_{2}$ become zero, while $\alpha_{3}$ can be nonzero.

The squared masses of the physical Higgs bosons are given by

$$
\begin{aligned}
m_{H_{5}^{ \pm \pm}}^{2} & =m_{H_{5}}^{2}-\frac{\nu}{v_{\Delta}}\left(\frac{v_{\phi}^{2}}{2} \lambda_{5}+12 \mu_{2} v_{\Delta}\right), \\
m_{H_{5}^{ \pm}}^{2} & =m_{H_{5}}^{2}+\frac{\nu}{8 v_{\Delta}}\left(64 v_{\Delta}^{2} \lambda_{3}+2 v_{\phi}^{2} \lambda_{5}+v_{\phi}^{2} \frac{\mu_{1}}{v_{\Delta}}\right)+\mathcal{O}\left(\nu^{2}\right), \\
m_{H_{5}^{0}}^{2} & =m_{H_{5}}^{2}+\frac{\nu}{6 v_{\Delta}}\left(64 v_{\Delta}^{2} \lambda_{3}+3 v_{\phi}^{2} \lambda_{5}+v_{\phi}^{2} \frac{\mu_{1}}{v_{\Delta}}+24 \mu_{2} v_{\Delta}\right)+\mathcal{O}\left(\nu^{2}\right), \\
m_{H_{3}^{ \pm}}^{2} & =m_{H_{3}}^{2}+\frac{\nu}{8 v_{\Delta}}\left(v_{\phi}^{2}-8 v_{\Delta}^{2}\right)\left(2 \lambda_{5}+\frac{\mu_{1}}{v_{\Delta}}\right)+\mathcal{O}\left(\nu^{2}\right), \\
m_{H_{3}^{0}}^{2} & =m_{H_{3}}^{2}-\frac{\nu}{2 v_{\Delta}}\left(v_{\phi}^{2}+8 v_{\Delta}^{2}\right) \lambda_{5} \\
m_{H_{1}}^{2} & =c_{\alpha_{3}}^{2} M_{11}^{2}+s_{\alpha_{3}}^{2} M_{22}^{2}+2 s_{\alpha_{3}} c_{\alpha_{3}} M_{12}^{2}+\mathcal{O}\left(\nu^{2}\right), \\
m_{h}^{2} & =s_{\alpha_{3}}^{2} M_{11}^{2}+c_{\alpha_{3}}^{2} M_{22}^{2}-2 s_{\alpha_{3}} c_{\alpha_{3}} M_{12}^{2}+\mathcal{O}\left(\nu^{2}\right),
\end{aligned}
$$

where

$$
\begin{aligned}
& m_{H_{5}}^{2}=8 v_{\Delta}^{2} \lambda_{3}-\frac{3}{2} v_{\phi}^{2} \lambda_{5}-\frac{v_{\phi}^{2}}{4} \frac{\mu_{1}}{v_{\Delta}}-12 v_{\Delta} \mu_{2}, \\
& m_{H_{3}}^{2}=-\frac{1}{4}\left(v_{\phi}^{2}+8 v_{\Delta}^{2}\right)\left(2 \lambda_{5}+\frac{\mu_{1}}{v_{\Delta}}\right),
\end{aligned}
$$

and

$$
\begin{aligned}
& M_{11}^{2}=8 v_{\Delta}^{2}\left(3 \lambda_{2}+\lambda_{3}\right)-v_{\phi}^{2} \frac{\mu_{1}}{4 v_{\Delta}}+6 v_{\Delta} \mu_{2}+\frac{\nu}{v_{\Delta}}\left[\frac{16}{3} v_{\Delta}^{2}\left(3 \lambda_{2}+\lambda_{3}\right)+v_{\phi}^{2} \frac{\mu_{1}}{12 v_{\Delta}}+2 v_{\Delta} \mu_{2}\right], \\
& M_{22}^{2}=8 v_{\phi}^{2} \lambda_{1}, \\
& M_{12}^{2}=\frac{\sqrt{3}}{2} v_{\phi}\left[4 v_{\Delta}\left(2 \lambda_{4}+\lambda_{5}\right)+\mu_{1}\right]+\frac{2 \sqrt{3}}{3} \nu v_{\phi}\left(2 \lambda_{4}+\lambda_{5}\right) .
\end{aligned}
$$


It is observed that in the $\nu \rightarrow 0$ limit, the different charged states within each multiplet have the same mass as the consequence of the restoration of the custodial symmetry.

\section{APPENDIX B: INTERACTION TERMS OF THE HIGGS BOSON}

We give expressions for the relevant three-point and four-point interaction terms of the Higgs bosons. The scalar-gaugegauge interaction terms are given by

$$
\begin{aligned}
\mathcal{L}_{S V V}= & \sum_{\varphi}\left[\frac{2 m_{W}^{2}}{v} c_{\varphi W W} \varphi W^{+\mu} W_{\mu}^{-}+\frac{m_{Z}^{2}}{v} c_{\varphi Z Z} \varphi Z^{\mu} Z_{\mu}\right] \\
& +\frac{2 m_{W}^{2}}{v}\left(\frac{c_{\beta}}{\sqrt{2}} H_{5}^{++} W^{-\mu} W_{\mu}^{-}-\frac{c_{\beta}}{c_{W}} H_{5}^{+} Z^{\mu} W_{\mu}-\frac{s_{W}^{2}}{c_{W}} G^{+} Z^{\mu} W_{\mu}^{-}+\text {H.c. }\right),
\end{aligned}
$$

where

$$
\begin{aligned}
c_{h V V} & =c_{\alpha} s_{\beta}-\frac{2 \sqrt{6}}{3} s_{\alpha} c_{\beta}, \quad c_{H_{1} V V}=s_{\alpha} s_{\beta}+\frac{2 \sqrt{6}}{3} c_{\alpha} c_{\beta}, \quad(V=W, Z), \\
c_{H_{5}^{0} W W} & =-\frac{c_{\beta}}{\sqrt{3}}, \quad c_{H_{5}^{0} Z Z}=\frac{2 c_{\beta}}{\sqrt{3}},
\end{aligned}
$$

and

$$
\sum_{\varphi} \equiv \sum_{\varphi=h, H_{1}, H_{5}^{0}}
$$

The scalar-scalar-gauge interaction terms are given by

$$
\begin{aligned}
\mathcal{L}_{S S V}= & i \frac{g}{2}\left[\sum_{\varphi} c_{\varphi W W}\left(\varphi \partial G^{+}\right)^{\mu}-\sum_{\varphi} c_{\varphi H_{3} W}\left(\varphi \partial H_{3}^{+}\right)^{\mu}\right. \\
& -i\left(G^{0} \partial G^{+}\right)^{\mu}-i\left(H_{3}^{0} \partial H_{3}^{+}\right)^{\mu} \\
& -i c_{\beta}\left(G^{0} \partial H_{5}^{+}\right)^{\mu}+i s_{\beta}\left(H_{3}^{0} \partial H_{5}^{+}\right)^{\mu}+\sqrt{3}\left(H_{5}^{0} \partial H_{5}^{+}\right)^{\mu} \\
& \left.+\sqrt{2} c_{\beta}\left(G^{-} \partial H_{5}^{++}\right)^{\mu}-\sqrt{2} s_{\beta}\left(H_{3}^{-} \partial H_{5}^{++}\right)^{\mu}+\sqrt{2}\left(H_{5}^{-} \partial H_{5}^{++}\right)^{\mu}\right] W_{\mu}^{-}+\text {H.c. }, \\
& +\frac{g_{Z}}{2}\left\{\sum_{\varphi} c_{\varphi Z Z}\left(\varphi \partial G^{0}\right)^{\mu}-\sum_{\varphi} c_{\varphi H_{3} Z}\left(\varphi \partial H_{3}^{0}\right)^{\mu}\right. \\
& -i c_{2 W}\left[\left(G^{+} \partial G^{-}\right)^{\mu}+\left(H_{3}^{+} \partial H_{3}^{-}\right)^{\mu}+\left(H_{5}^{+} \partial H_{5}^{-}\right)^{\mu}+2\left(H_{5}^{++} \partial H_{5}^{--}\right)^{\mu}\right] \\
& \left.+i c_{\beta}\left(H_{5}^{+} \partial G^{-}\right)-i s_{\beta}\left(H_{5}^{+} \partial H_{3}^{-}\right)\right\} Z_{\mu},
\end{aligned}
$$

where $(A \partial B)^{\mu} \equiv A\left(\partial^{\mu} B\right)-\left(\partial^{\mu} A\right) B, c_{2 W} \equiv c_{W}^{2}-s_{W}^{2}$ and

$$
\begin{aligned}
c_{h H_{3} V} & =-c_{\alpha} c_{\beta}-\frac{2 \sqrt{6}}{3} s_{\alpha} s_{\beta}, \\
c_{H_{1} H_{3} V} & =-s_{\alpha} c_{\beta}+\frac{2 \sqrt{6}}{3} c_{\alpha} s_{\beta}, \quad(V=W, Z), \\
c_{H_{5}^{0} H_{3} W} & =-\frac{s_{\beta}}{\sqrt{3}}, \quad c_{H_{5}^{0} H_{3} Z}=\frac{2 s_{\beta}}{\sqrt{3}} .
\end{aligned}
$$


The scalar-scalar-gauge-gauge interaction terms are given by

$\mathcal{L}_{S S V V}=\sum_{\varphi}\left(\frac{g^{2}}{4} c_{h \varphi W W} W^{+\mu} W_{\mu}^{-} h \varphi+\frac{g_{Z}^{2}}{8} c_{h \varphi Z Z} Z^{\mu} Z_{\mu} h \varphi\right)$

where

$$
\begin{array}{rlrl}
c_{h h V V} & =\frac{11-5 c_{2 \alpha}}{6}, & c_{h H_{1} V V}=-\frac{5}{3} s_{2 \alpha}, & (V=W, Z) \\
c_{h H_{5} W W} & =\frac{4}{3} \sqrt{2} s_{\alpha}, & c_{h H_{5} Z Z}=-\frac{8}{3} \sqrt{2} s_{\alpha}, \\
c_{H_{1} H_{1} V V} & =\frac{11+5 c_{2 \alpha}}{6}, & c_{H_{5}^{0} H_{5}^{0} W W}=\frac{10}{3} .
\end{array}
$$

The Yukawa interaction terms for the third-generation fermions are given by

$$
\begin{aligned}
\mathcal{L}_{f f S}= & -\sum_{f=t, b, \tau} \frac{m_{f}}{v}\left(c_{h f f} \bar{f} f h+c_{H_{1} f f} \bar{f} f H_{1}-2 i I_{f} \cot \beta \bar{f} \gamma_{5} f H_{3}^{0}\right) \\
& -\frac{\sqrt{2}}{v} \cot \beta\left[\bar{t}\left(m_{b} P_{R}-m_{t} P_{L}\right) b H_{3}^{+}+\bar{\nu}_{\tau} m_{\tau} P_{R} \tau H_{3}^{+}+\text {H.c. }\right],
\end{aligned}
$$

where $I_{t}=1 / 2$ and $I_{b}=I_{\tau}=-1 / 2$, and

$$
c_{h f f}=\frac{c_{\alpha}}{s_{\beta}}, \quad c_{H_{1} f f}=\frac{s_{\alpha}}{s_{\beta}} .
$$

\section{APPENDIX C: 1PI CONTRIBUTIONS}

We give the analytic expressions for 1PI diagram contributions that appear in the renormalized Higgs boson vertices. Section C 1 defines the required loop functions. The formulas for the 1PI diagram contributions to one-, two-, and three-point functions are given in Secs. C 2-C 4, respectively. Calculations are performed in the 't HooftFeynman gauge, where the masses of the NG bosons $m_{G^{ \pm}}$ and $m_{G^{0}}$ become $m_{W}$ and $m_{Z}$, respectively.

\section{Loop functions}

In order to systematically express all one-loop amplitudes, we introduce the Passarino-Veltman one-, two-, and three-point functions [39] as follows:

$$
\frac{i}{16 \pi^{2}}\left[C_{0}, C^{\mu}, C^{\mu \nu}\right]\left(p_{1}^{2}, p_{2}^{2},\left(p_{1}+p_{2}\right)^{2} ; m_{1}, m_{2}, m_{3}\right)=\mu^{4-D} \int \frac{d^{D} k}{(2 \pi)^{D}} \frac{\left[1, k^{\mu}, k^{\mu} k^{\nu}\right]}{D_{1} D_{2} D_{3}},
$$

$$
\frac{i}{16 \pi^{2}}\left[B_{0}, B^{\mu}, B^{\mu \nu}\right]\left(p_{1}^{2} ; m_{1}, m_{2}\right)=\mu^{4-D} \int \frac{d^{D} k}{(2 \pi)^{D}} \frac{\left[1, k^{\mu}, k^{\mu} k^{\nu}\right]}{D_{1} D_{2}}
$$

where $D=4-2 \epsilon$, and $\mu$ is a dimensionful parameter. The functions in the denominators, $D_{1,2,3}$, are defined by

$$
D_{1}=k^{2}-m_{1}^{2}+i \varepsilon, \quad D_{2}=\left(k+p_{1}\right)^{2}-m_{2}^{2}+i \varepsilon, \quad D_{3}=\left(k+p_{1}+p_{2}\right)^{2}-m_{3}^{2}+i \varepsilon .
$$

The $B$ and $C$ tensor functions are decomposed into the following forms in terms of scalar coefficients $B_{1,21,22}$ and $C_{11,12,21,22,23,24}$ :

$$
\begin{gathered}
B^{\mu}=p_{1}^{\mu} B_{1}, \\
B^{\mu \nu}=p_{1}^{\mu} p_{1}^{\nu} B_{21}+g^{\mu \nu} B_{22}, \\
C^{\mu}=p_{1}^{\mu} C_{11}+p_{2}^{\mu} C_{12}, \\
C^{\mu \nu}=p_{1}^{\mu} p_{1}^{\nu} C_{21}+p_{2}^{\mu} p_{2}^{\nu} C_{22}+\left(p_{1}^{\mu} p_{2}^{\nu}+p_{1}^{\nu} p_{2}^{\mu}\right) C_{23}+g^{\mu \nu} C_{24} .
\end{gathered}
$$

It is convenient to define coefficients $\lambda_{\phi_{i} \phi_{j} \phi_{k}}$ and $\lambda_{\phi_{i} \phi_{j} \phi_{k} \phi_{l}}$, respectively, for the three-point and four-point scalar interaction terms as

$$
\mathcal{L}=+\lambda_{\phi_{i} \phi_{j} \phi_{k}} \phi_{i} \phi_{j} \phi_{k}+\lambda_{\phi_{i} \phi_{j} \phi_{k} \phi_{l}} \phi_{i} \phi_{j} \phi_{k} \phi_{l}+\cdots
$$


As some of these coefficients are proportional to each other, we thus define the following quantities:

$$
\begin{aligned}
\lambda_{H_{5} H_{5} \phi} & \equiv \lambda_{H_{5}^{0} H_{5}^{0} \phi}=\frac{1}{2} \lambda_{H_{5}^{+} H_{5}^{-} \phi}=\frac{1}{2} \lambda_{H_{5}^{++} H_{5}^{--} \phi}, \\
\lambda_{H_{3} H_{3} \phi} & \equiv \lambda_{H_{3}^{0} H_{3}^{0} \phi}=\frac{1}{2} \lambda_{H_{3}^{+} H_{3}^{-} \phi}, \\
\lambda_{G G \phi} & \equiv \lambda_{G^{0} G^{0} \phi}=\frac{1}{2} \lambda_{G^{+} G^{-} \phi}, \\
\lambda_{G G H_{5}^{0}} & \equiv \lambda_{G^{0} G^{0} H_{5}^{0}}=-\lambda_{G^{+} G^{-} H_{5}^{0}}, \\
\lambda_{H_{3} G \phi} & \equiv \lambda_{H_{3}^{0} G^{0} \phi}=\lambda_{H_{3}^{ \pm} G^{\mp} \phi}, \\
\lambda_{H_{3} G H_{5}^{0}} & \equiv \lambda_{H_{3}^{0} G^{0} H_{5}^{0}}=-2 \lambda_{H_{3}^{ \pm} G^{\mp} H_{5}^{0}},
\end{aligned}
$$

and

$$
\begin{aligned}
\lambda_{H_{5} H_{5} h h} & \equiv \lambda_{H_{5}^{++} H_{5}^{--} h h}=\lambda_{H_{5}^{+} H_{5}^{-} h h}=2 \lambda_{H_{5}^{0} H_{5}^{0} h h}, \\
\lambda_{H_{3} H_{3} h h} & \equiv \lambda_{H_{3}^{+} H_{3}^{-} h h}=2 \lambda_{H_{3}^{0} H_{3}^{0} h h}, \\
\lambda_{G G h h} & \equiv \lambda_{G^{+} G^{-} h h}=2 \lambda_{G^{0} G^{0} h h}, \\
\lambda_{H_{3} G h h} & \equiv \lambda_{H_{3}^{+} G^{-} h h}=\lambda_{H_{3}^{0} G^{0} h h},
\end{aligned}
$$

with $\phi=h$ or $H_{1}$.

\section{One-point functions}

The 1PI diagram contributions to one-point functions for $h, H_{1}$ and $H_{5}^{0}$ are given by

$$
\begin{aligned}
T_{h}^{1 \mathrm{PI}}= & -\sum_{f=t, b, \tau} \frac{N_{c}^{f}}{16 \pi^{2}} \frac{4 m_{f}^{2}}{v} c_{h f f} A\left(m_{f}\right) \\
& -\frac{1}{16 \pi^{2}}\left\{5 \lambda_{H_{5} H_{5}} A\left(m_{H_{5}}\right)+3 \lambda_{H_{3} H_{3} h} A\left(m_{H_{3}}\right)+\lambda_{H_{1} H_{1} h} A\left(m_{H_{1}}\right)\right. \\
& +3 \lambda_{h h h} A\left(m_{h}\right)-\lambda_{G G h}\left[2 A\left(m_{G^{ \pm}}\right)+A\left(m_{G^{0}}\right)\right] \\
& \left.-3 c_{h V V}\left[g m_{W} A\left(m_{W}\right)+\frac{g_{Z}}{2} m_{Z} A\left(m_{Z}\right)-\frac{2}{3} g m_{W}^{3}-\frac{g_{Z}}{3} m_{Z}^{3}\right]\right\}, \\
T_{H_{1}}^{1 \mathrm{PI}}=- & \sum_{f=t, b, \tau} \frac{N_{c}^{f}}{16 \pi^{2}} \frac{4 m_{f}^{2}}{v} c_{H_{1} f f} A\left(m_{f}\right) \\
- & \frac{1}{16 \pi^{2}}\left\{5 \lambda_{H_{5} H_{5} H_{1}} A\left(m_{H_{5}}\right)+3 \lambda_{H_{3} H_{3} H_{1}} A\left(m_{H_{3}}\right)+3 \lambda_{H_{1} H_{1} H_{1}} A\left(m_{H_{1}}\right)\right. \\
+ & \lambda_{H_{1} h h} A\left(m_{h}\right)-\lambda_{G G H_{1}}\left[2 A\left(m_{G^{ \pm}}\right)+A\left(m_{G^{0}}\right)\right] \\
- & \left.3 c_{H_{1} V V}\left[g m_{W} A\left(m_{W}\right)+\frac{g_{Z}}{2} m_{Z} A\left(m_{Z}\right)-\frac{2}{3} g m_{W}^{3}-\frac{g_{Z}}{3} m_{Z}^{3}\right]\right\}, \\
T_{H_{5}^{0}}^{1 \mathrm{PI}}= & \frac{1}{16 \pi^{2}}\left\{\lambda_{G G H_{5}^{0}}\left[A\left(m_{G^{ \pm}}\right)-A\left(m_{G^{0}}\right)\right]\right. \\
+ & \left.g m_{W} c_{H_{5}^{0} W W}\left[3 A\left(m_{W}\right)-2 m_{W}^{2}\right]+\frac{g_{Z}}{2} m_{Z} c_{H_{5}^{0} Z Z}\left[3 A\left(m_{Z}\right)-2 m_{Z}^{2}\right]\right\},
\end{aligned}
$$

where $N_{c}^{f}=3(1)$ for $f=t, b(\tau)$. We note that for $T_{H_{5}^{0}}^{1 \mathrm{P}}$, the 5-plet and 3-plet Higgs boson loop contributions are cancelled among themselves. 


\section{Two-point functions}

The 1PI diagram contributions to two-point functions for CP-even Higgs bosons are given by

$$
\begin{aligned}
& \Pi_{h h}^{1 \mathrm{PI}}\left(q^{2}\right)=\Pi_{h h}^{f f}\left(q^{2}\right)+\Pi_{h h}^{S V+V V}\left(q^{2}\right) \\
& +\frac{1}{16 \pi^{2}}\left\{10 \lambda_{H_{5} H_{5} h}^{2} B_{0}\left(q^{2} ; m_{H_{5}}, m_{H_{5}}\right)+6 \lambda_{H_{3} H_{3} h}^{2} B_{0}\left(q^{2} ; m_{H_{3}}, m_{H_{3}}\right)\right. \\
& +18 \lambda_{h h h}^{2} B_{0}\left(q^{2} ; m_{h}, m_{h}\right)+2 \lambda_{H_{1} H_{1} h}^{2} B_{0}\left(q^{2} ; m_{H_{1}}, m_{H_{1}}\right)+4 \lambda_{H_{1} h h}^{2} B_{0}\left(q^{2} ; m_{H_{1}}, m_{h}\right) \\
& +\lambda_{H_{3} G h}^{2}\left[2 B_{0}\left(q^{2} ; m_{G^{ \pm}}, m_{H_{3}}\right)+B_{0}\left(q^{2} ; m_{G^{0}}, m_{H_{3}}\right)\right] \\
& \left.+2 \lambda_{G G h}^{2}\left[2 B_{0}\left(q^{2} ; m_{G^{ \pm}}, m_{G^{ \pm}}\right)+B_{0}\left(q^{2} ; m_{G^{0}}, m_{G^{0}}\right)\right]\right\} \\
& -\frac{2}{16 \pi^{2}} \sum_{X=\text { scalars }}\left(1+5 \delta_{X h}\right) \lambda_{X X^{*} h h} A\left(m_{X}\right) \text {, } \\
& \Pi_{H_{1} h}^{1 \mathrm{PI}}\left(q^{2}\right)=\Pi_{H_{1} h}^{f f}\left(q^{2}\right)+\Pi_{H_{1} h}^{S V+V V}\left(q^{2}\right) \\
& +\frac{1}{16 \pi^{2}}\left[10 \lambda_{H_{5} H_{5} h} \lambda_{H_{5} H_{5} H_{1}} B_{0}\left(q^{2} ; m_{H_{5}}, m_{H_{5}}\right)+6 \lambda_{H_{3} H_{3} h} \lambda_{H_{3} H_{3} H_{1}} B_{0}\left(q^{2} ; m_{H_{3}}, m_{H_{3}}\right)\right. \\
& +6 \lambda_{h h h} \lambda_{H_{1} h h} B_{0}\left(q^{2} ; m_{h}, m_{h}\right)+6 \lambda_{H_{1} H_{1} h} \lambda_{H_{1} H_{1} H_{1}} B_{0}\left(q^{2} ; m_{H_{1}}, m_{H_{1}}\right) \\
& +4 \lambda_{H_{1} h h} \lambda_{H_{1} H_{1} h} B_{0}\left(q^{2} ; m_{H_{1}}, m_{h}\right) \\
& +\lambda_{H_{3} G h} \lambda_{H_{3} G H_{1}}\left[2 B_{0}\left(q^{2} ; m_{G^{ \pm}}, m_{H_{3}}\right)+B_{0}\left(q^{2} ; m_{G^{0}}, m_{H_{3}}\right)\right] \\
& +2 \lambda_{G G h} \lambda_{G G H_{1}}\left[2 B_{0}\left(q^{2} ; m_{G^{ \pm}}, m_{G^{ \pm}}\right)+B_{0}\left(q^{2} ; m_{G^{0}}, m_{G^{0}}\right)\right] \\
& -\frac{1}{16 \pi^{2}} \sum_{X=\text { scalars }}\left(1+2 \delta_{X h}+2 \delta_{X H_{1}}\right) \lambda_{X X^{*} H_{1} h} A\left(m_{X}\right) \text {, } \\
& \Pi_{H_{5}^{0} h}^{1 \mathrm{PI}}\left(q^{2}\right)=\Pi_{H_{5}^{0} h}^{S V+V V}\left(q^{2}\right) \\
& -\frac{1}{16 \pi^{2}}\left\{\lambda_{H_{3} G h} \lambda_{H_{3} G H_{5}^{0}}\left[B_{0}\left(q^{2} ; m_{G^{ \pm}}, m_{H_{3}}\right)-B_{0}\left(q^{2} ; m_{G^{0}}, m_{H_{3}}\right)\right]\right. \\
& \left.+2 \lambda_{G G h} \lambda_{G G H_{5}^{0}}\left[B_{0}\left(q^{2} ; m_{G^{ \pm}}, m_{G^{ \pm}}\right)-B_{0}\left(q^{2} ; m_{G^{0}}, m_{G^{0}}\right)\right]\right\} \\
& -\frac{1}{16 \pi^{2}} \sum_{X=\text { scalars }}\left(1+2 \delta_{X h}+2 \delta_{X H_{1}}\right) \lambda_{X X^{*} H_{3}^{0} G^{0}} A\left(m_{X}\right) \text {, }
\end{aligned}
$$

where

$$
\begin{aligned}
\Pi_{\varphi h}^{f f}\left(q^{2}\right)= & -\frac{1}{16 \pi^{2}} \sum_{f=t, b, \tau} \frac{4 m_{f}^{2} N_{c}^{f}}{v^{2}} c_{h f f} c_{\varphi f f}\left[A\left(m_{f}\right)+\left(2 m_{f}^{2}-\frac{q^{2}}{2} B_{0}\left(q^{2} ; m_{f}, m_{f}\right)\right)\right], \\
\Pi_{\varphi h}^{S V+V V}\left(q^{2}\right)= & -\frac{1}{16 \pi^{2}} \frac{2 m_{W}^{2}}{v^{2}}\left\{c_{h W W} c_{\varphi W W}\left[\left(2 q^{2}-6 m_{W}^{2}\right) B_{0}\left(q^{2} ; m_{W}, m_{W}\right)+A\left(m_{W}\right)+4 m_{W}^{2}\right]\right. \\
& +\frac{c_{h Z Z} c_{\varphi Z Z}}{2 c_{W}^{2}}\left[\left(2 q^{2}-6 m_{Z}^{2}\right) B_{0}\left(q^{2} ; m_{Z}, m_{Z}\right)+A\left(m_{Z}\right)+4 m_{Z}^{2}\right] \\
& +c_{h H_{3} W} c_{\varphi H_{3} W}\left[\left(2 q^{2}+2 m_{H_{3}}^{2}-m_{W}^{2}\right) B_{0}\left(q^{2} ; m_{H_{3}}, m_{W}\right)+2 A\left(m_{W}\right)-A\left(m_{H_{3}}\right)\right] \\
& \left.+\frac{c_{h H_{3} Z} c_{\varphi H_{3} Z}}{2 c_{W}^{2}}\left[\left(2 q^{2}+2 m_{H_{3}}^{2}-m_{Z}^{2}\right) B_{0}\left(q^{2} ; m_{H_{3}}, m_{Z}\right)+2 A\left(m_{Z}\right)-A\left(m_{H_{3}}\right)\right]\right\} \\
& +\left(1+\delta_{\varphi h}\right) \frac{1}{16 \pi^{2}}\left\{g^{2} c_{h \varphi W W}\left[A\left(m_{W}\right)-\frac{m_{W}^{2}}{2}\right]+\frac{g_{Z}^{2}}{2} c_{h \varphi Z Z}\left[A\left(m_{Z}\right)-\frac{m_{Z}^{2}}{2}\right]\right\} .
\end{aligned}
$$

That for the $H_{3}^{0}-G^{0}$ mixing is expressed as 


$$
\begin{aligned}
\Pi_{H_{3}^{0} G^{0}}^{1 \mathrm{PI}}\left(q^{2}\right)= & -\frac{1}{16 \pi^{2}} \sum_{f=t, b, \tau} \frac{4 m_{f}^{2} N_{c}^{f}}{v^{2}} \cot \beta\left[A\left(m_{f}\right)-\frac{q^{2}}{2} B_{0}\left(q^{2} ; m_{f}, m_{f}\right)\right] \\
& +\frac{1}{16 \pi^{2}}\left\{\frac{g^{2}}{2} s_{\beta} c_{\beta}\left[\left(2 q^{2}+2 m_{H_{5}}^{2}-m_{W}^{2}\right) B_{0}\left(q^{2} ; m_{H_{5}}, m_{W}\right)+2 A\left(m_{W}\right)-A\left(m_{H_{5}}\right)\right]\right. \\
& \left.+\frac{g_{Z}^{2}}{4} \sum_{\varphi} c_{\varphi H_{3} Z} c_{\varphi Z Z}\left[\left(2 q^{2}+2 m_{\varphi}^{2}-m_{Z}^{2}\right) B_{0}\left(q^{2} ; m_{\varphi}, m_{Z}\right)+2 A\left(m_{Z}\right)-A\left(m_{\varphi}\right)\right]\right\} \\
& -\frac{1}{16 \pi^{2}} s_{\beta} c_{\beta}\left[2 g^{2} A\left(m_{W}\right)+3 g_{Z}^{2} A\left(m_{Z}\right)-g^{2} m_{W}^{2}-\frac{3 g_{Z}^{2}}{2} m_{Z}^{2}\right] \\
& +\frac{1}{16 \pi^{2}}\left\{3 \lambda_{H_{3} G H_{5}^{0}}\left[\lambda_{H_{5}^{0} H_{3}^{0} H_{3}^{0}} B_{0}\left(q^{2} ; m_{H_{5}}, m_{H_{3}}\right)+\lambda_{G G H_{5}^{0}} B_{0}\left(q^{2} ; m_{H_{5}}, m_{G^{ \pm}}\right)\right]\right. \\
& \left.+2 \sum_{\varphi}\left[\lambda_{H_{3} H_{3} \varphi} \lambda_{H_{3} G \varphi} B_{0}\left(q^{2} ; m_{\varphi}, m_{H_{3}}\right)+\lambda_{G G \varphi} \lambda_{H_{3} G \varphi} B_{0}\left(q^{2} ; m_{\varphi}, m_{G^{0}}\right)\right]\right\} \\
& -\frac{1}{16 \pi^{2}} \sum_{X=\text { scalar }}\left(1+2 \delta_{X G^{0}}+2 \delta_{X H_{3}^{0}}\right) \lambda_{X X^{*} H_{3}^{0} G^{0}} A\left(m_{X}\right) .
\end{aligned}
$$

Next, fermion 2-point functions can be decomposed into the following three parts:

$$
\Pi_{f f}^{1 \mathrm{PI}}\left(q^{2}\right)=q \Pi_{f f, V}^{1 \mathrm{PI}}\left(q^{2}\right)-q \gamma_{5} \Pi_{f f, A}^{1 \mathrm{PI}}\left(q^{2}\right)+m_{f} \Pi_{f f, S}^{1 \mathrm{PI}}\left(q^{2}\right)
$$

Each part subtracted by the SM contribution is calculated as

$$
\begin{aligned}
& \Delta \Pi_{f f, V}^{1 \mathrm{PI}}\left(q^{2}\right)=-\frac{1}{16 \pi^{2}} \frac{m_{f}^{2}}{v^{2}}\left[\left(c_{h f f}^{2}-1\right) B_{1}\left(q^{2} ; m_{f}, m_{h}\right)+c_{H_{1} f f}^{2} B_{1}\left(q^{2} ; m_{f}, m_{H_{1}}\right)\right. \\
&\left.+\cot ^{2} \beta B_{1}\left(q^{2} ; m_{f}, m_{H_{3}}\right)+\left(1+\frac{m_{f^{\prime}}^{2}}{m_{f}^{2}}\right) \cot ^{2} \beta B_{1}\left(q^{2} ; m_{f^{\prime}}, m_{H_{3}}\right)\right], \\
& \Delta \Pi_{f f, A}^{\mathrm{PI}}\left(q^{2}\right)=\frac{1}{16 \pi^{2}} \frac{m_{f}^{2}-m_{f^{\prime}}^{2}}{v^{2}} \cot ^{2} \beta B_{1}\left(q^{2} ; m_{f^{\prime}}, m_{H_{3}}\right), \\
& \Delta \Pi_{f f, S}^{1 \mathrm{PI}}\left(q^{2}\right)= \frac{1}{16 \pi^{2}} \frac{m_{f}^{2}}{v^{2}}\left[\left(c_{h f f}^{2}-1\right) B_{0}\left(q^{2} ; m_{f}, m_{h}\right)+c_{H_{1} f f}^{2} B_{0}\left(q^{2} ; m_{f}, m_{H_{1}}\right)\right. \\
&\left.-\cot ^{2} \beta B_{0}\left(q^{2} ; m_{f}, m_{H_{3}}\right)-2 \frac{m_{f^{\prime}}^{2}}{m_{f}^{2}} \cot ^{2} \beta B_{0}\left(q^{2} ; m_{f^{\prime}}, m_{H_{3}}\right)\right]
\end{aligned}
$$

where $v_{f}$ and $a_{f}$ are the coefficients of the vector coupling and axial-vector coupling of the $Z f \bar{f}$ vertex, given by

$$
v_{f}=\frac{I_{f}}{2}-s_{W}^{2} Q_{f}, \quad a_{f}=\frac{I_{f}}{2},
$$

with $Q_{f}$ being the electric charge of the fermion $f$. In addition, $m_{f^{\prime}}$ is the mass of fermion $f^{\prime}$ with an opposite weak isospin to $f$.

Finally, we present the expressions for the transverse components of the gauge boson two-point functions. Each of the functions subtracted by the SM contribution is given by 


$$
\begin{aligned}
& \Delta \Pi_{W W}^{1 \mathrm{PI}}\left(q^{2}\right)=\frac{g^{2}}{64 \pi^{2}}\left\{5 B_{5}\left(q^{2} ; m_{H_{5}}, m_{H_{5}}\right)+3 s_{\beta}^{2} B_{5}\left(q^{2} ; m_{H_{5}}, m_{H_{3}}\right)+B_{5}\left(q^{2} ; m_{H_{3}}, m_{H_{3}}\right)\right. \\
& +2 c_{\beta}^{2} B_{5}\left(q^{2} ; m_{H_{5}}, m_{G^{ \pm}}\right)+c_{\beta}^{2} B_{5}\left(q^{2} ; m_{H_{5}}, m_{G^{0}}\right) \\
& \left.+\sum_{\varphi}\left[c_{\varphi H_{3} W}^{2} B_{5}\left(q^{2} ; m_{H_{3}}, m_{\varphi}\right)+c_{\varphi W W}^{2} B_{5}\left(q^{2} ; m_{G^{ \pm}}, m_{\varphi}\right)\right]-B_{5}\left(q^{2} ; m_{G^{ \pm}}, m_{h}\right)\right\} \\
& +\frac{g^{2} m_{W}^{2}}{16 \pi^{2}}\left[2 c_{\beta}^{2} B_{0}\left(q^{2} ; m_{H_{5}}, m_{W}\right)+\frac{c_{\beta}^{2}}{c_{W}^{2}} B_{0}\left(q^{2} ; m_{H_{5}}, m_{Z}\right)\right. \\
& \left.+\sum_{\varphi} c_{\varphi W W}^{2} B_{0}\left(q^{2} ; m_{\varphi}, m_{W}\right)-B_{0}\left(q^{2} ; m_{h}, m_{W}\right)\right] \\
& \Delta \Pi_{Z Z}^{1 \mathrm{PI}}\left(q^{2}\right)=\frac{g_{Z}^{2}}{64 \pi^{2}}\left\{5 c_{2 W}^{2} B_{5}\left(q^{2} ; m_{H_{5}}, m_{H_{5}}\right)+c_{2 W}^{2} B_{5}\left(q^{2} ; m_{H_{3}}, m_{H_{3}}\right)\right. \\
& +2 s_{\beta}^{2} B_{5}\left(q^{2} ; m_{H_{5}}, m_{H_{3}}\right)+2 c_{\beta}^{2} B_{5}\left(q^{2} ; m_{H_{5}}, m_{G^{ \pm}}\right) \\
& \left.+\sum_{\varphi}\left[c_{\varphi H_{3}^{0} Z}^{2} B_{5}\left(q^{2} ; m_{H_{3}}, m_{\varphi}\right)+c_{\varphi Z Z}^{2} B_{5}\left(q^{2} ; m_{\varphi}, m_{G^{0}}\right)\right]-B_{5}\left(q^{2} ; m_{h}, m_{G^{0}}\right)\right\} \\
& +\frac{g_{Z}^{2} m_{Z}^{2}}{16 \pi^{2}}\left[2 c_{\beta}^{2} c_{W}^{2} B_{0}\left(q^{2} ; m_{H_{5}}, m_{W}\right)+\sum_{\varphi} c_{\varphi Z Z}^{2} B_{0}\left(q^{2} ; m_{\varphi}, m_{Z}\right)-B_{0}\left(q^{2} ; m_{h}, m_{Z}\right)\right] \text {, } \\
& \Delta \Pi_{Z \gamma}^{1 \mathrm{PI}}\left(q^{2}\right)=\frac{e g_{Z}}{32 \pi^{2}} c_{2 W}\left[5 B_{5}\left(q^{2} ; m_{H_{5}}, m_{H_{5}}\right)+B_{5}\left(q^{2} ; m_{H_{3}}, m_{H_{3}}\right)\right] \text {, } \\
& \Delta \Pi_{\gamma \gamma}^{1 \mathrm{PI}}\left(q^{2}\right)=\frac{e^{2}}{16 \pi^{2}}\left[5 B_{5}\left(q^{2} ; m_{H_{5}}, m_{H_{5}}\right)+B_{5}\left(q^{2} ; m_{H_{3}}, m_{H_{3}}\right)\right],
\end{aligned}
$$

where $B_{5}\left(q^{2} ; m_{1}, m_{2}\right) \equiv A\left(m_{1}\right)+A\left(m_{2}\right)-4 B_{22}\left(q^{2} ; m_{1}, m_{2}\right)$.

\section{Three-point functions}

For the 1PI diagram contributions to three-point functions, we use a shorthand notation for the Passarino-Veltman's $C$ functions,

$$
C_{i, i k}(A, B, C) \equiv C_{i, i k}\left(p_{1}^{2}, p_{2}^{2}, q^{2} ; m_{A}, m_{B}, m_{C}\right)
$$

where $p_{1}^{\mu}$ and $p_{2}^{\mu}$ are incoming four-momenta of gauge bosons, fermions and (on-shell) Higgs bosons for the $h V V, h f f$, and $h h h$ vertices, respectively, and $q^{\mu}$ is the outgoing momentum of the Higgs boson. For the $h V V$ and $h f f$ vertices, we show the expressions corresponding to the first form factor defined in Eqs. (61) and (65), respectively. First, the 1PI diagram contributions to the $h V V$ vertices are

$$
\begin{aligned}
\Gamma_{h W W}^{1,1 \mathrm{PI}}\left(p_{1}^{2}, p_{2}^{2}, q^{2}\right)= & -\frac{3 g^{2} m_{t}^{2}}{16 \pi^{2} v} c_{h f f}\left[4 C_{24}(t, b, t)-\frac{1}{2} B_{0}\left(p_{2}^{2} ; m_{t}, m_{b}\right)-B_{0}\left(q^{2} ; m_{t}, m_{t}\right)-\frac{1}{2} B_{0}\left(p_{1}^{2} ; m_{t}, m_{b}\right)\right. \\
& \left.-\frac{1}{2}\left(2 m_{t}^{2}+2 m_{b}^{2}-p_{1}^{2}-p_{2}^{2}\right) C_{0}(t, b, t)\right]+\left(m_{t} \leftrightarrow m_{b}\right) \\
& +\frac{g^{3} m_{W}}{16 \pi^{2}}\left\{c_{h W W}\left[C_{h V V}^{V V V}(Z, W, Z)+c_{W}^{2} C_{h V V}^{V V V}(W, Z, W)+s_{W}^{2} C_{h V V}^{V V V}(W, \gamma, W)\right]\right. \\
& -\frac{s_{W}^{2}}{2} c_{h W W}\left[C_{h V V}^{S V V}\left(G^{ \pm}, Z, W\right)+C_{h V V}^{V V S}\left(W, Z, G^{ \pm}\right)-C_{h V V}^{S V V}\left(G^{ \pm}, \gamma, W\right)-C_{h V V}^{V V S}\left(W, \gamma, G^{ \pm}\right)\right] \\
& -m_{W}^{2} c_{h W W}\left[\sum_{\varphi} c_{\varphi V V}^{2} C_{0}(W, \varphi, W)+t_{W}^{4} C_{0}\left(Z, G^{ \pm}, Z\right)\right. \\
& \left.+\frac{c_{\beta}^{2}}{c_{W}^{4}} C_{0}\left(Z, H_{5}^{ \pm}, Z\right)+2 c_{\beta}^{2} C_{0}\left(W, H_{5}^{ \pm \pm}, W\right)\right]
\end{aligned}
$$


$+\frac{1}{2} \sum_{\varphi}\left[c_{h W W} c_{\varphi W W}^{2} \tilde{C}_{24}\left(G^{ \pm}, \varphi, W\right)+c_{h H_{3} W} c_{\varphi H_{3} W} c_{\varphi W W} \tilde{C}_{24}\left(H_{3}^{ \pm}, \varphi, W\right)\right]$

$+c_{\beta}^{2} c_{h W W} \tilde{C}_{24}\left(G^{ \pm}, H_{5}^{ \pm \pm}, W\right)+s_{\beta} c_{\beta} c_{h H_{3} V} \tilde{C}_{24}\left(H_{3}^{ \pm}, H_{5}^{ \pm \pm}, W\right)$

$+\frac{t_{W}^{2}}{2} c_{h V V} \tilde{C}_{24}\left(G^{0}, G^{ \pm}, Z\right)+\frac{c_{\beta}^{2}}{2 c_{W}^{2}} c_{h V V} \tilde{C}_{24}\left(G^{0}, H_{5}^{ \pm}, Z\right)+\frac{s_{\beta} c_{\beta}}{2 c_{W}^{2}} c_{h H_{3} V} \tilde{C}_{24}\left(H_{3}^{0}, H_{5}^{ \pm}, Z\right)$

$-c_{h V V}\left[3 B_{0}\left(q^{2}, m_{W}, m_{W}\right)+3 B_{0}\left(q^{2}, m_{Z}, m_{Z}\right)-4\right]$

$-\frac{1}{4} \sum_{\varphi}\left(1+\delta_{h \varphi}\right) c_{h \varphi W W} c_{\varphi W W} \tilde{B}_{0}(W, \varphi)-\frac{s_{W}^{2}}{2} c_{h W W}\left[t_{W}^{2} \tilde{B}_{0}\left(Z, G^{ \pm}\right)+\tilde{B}_{0}\left(\gamma, G^{ \pm}\right)\right]$

$\left.+\frac{\sqrt{3}}{2} c_{\beta} c_{h H_{5} W W}\left[\tilde{B}_{0}\left(W, H_{5}^{ \pm \pm}\right)+\frac{1}{2 c_{W}^{2}} \tilde{B}_{0}\left(Z, H_{5}^{ \pm}\right)\right]\right\}$

$+\frac{g^{2} m_{W}^{2}}{16 \pi^{2}}\left\{6 \lambda_{h h h} c_{h W W}^{2} C_{0}(h, W, h)+2 \lambda_{H_{1} H_{1} h} c_{H_{1} W W}^{2} C_{0}\left(H_{1}, W, H_{1}\right)\right.$

$+2 \lambda_{H_{1} h h} c_{h W W} c_{H_{1} W W} \tilde{C}_{0}\left(h, W, H_{1}\right)$

$+2 \lambda_{H_{5} H_{5} h}\left[c_{H_{5}^{0} W W}^{2} C_{0}\left(H_{5}^{0}, W, H_{5}^{0}\right)+2 c_{\beta}^{2} C_{0}\left(H_{5}^{ \pm \pm}, W, H_{5}^{ \pm \pm}\right)+\frac{c_{\beta}^{2}}{c_{W}^{2}} C_{0}\left(H_{5}^{ \pm}, Z, H_{5}^{ \pm}\right)\right]$

$\left.+2 \lambda_{G G h} s_{W}^{2}\left[C_{0}\left(G^{ \pm}, \gamma, G^{ \pm}\right)+t_{W}^{2} C_{0}\left(G^{ \pm}, Z, G^{ \pm}\right)\right]\right\}$

$-\frac{g^{2}}{16 \pi^{2}}\left\{2 \lambda_{G G h}\left[\sum_{\varphi} c_{\varphi W W}^{2} C_{24}\left(G^{ \pm}, \varphi, G^{ \pm}\right)+C_{24}\left(G^{ \pm}, G^{0}, G^{ \pm}\right)+2 c_{\beta}^{2} C_{24}\left(G^{ \pm}, H_{5}^{ \pm \pm}, G^{ \pm}\right)\right.\right.$

$\left.+C_{24}\left(G^{0}, G^{ \pm}, G^{0}\right)+c_{\beta}^{2} C_{24}\left(G^{0}, H_{5}^{ \pm}, G^{0}\right)\right]$

$+2 \lambda_{H_{3} H_{3} h}\left[\sum_{\varphi} c_{\varphi H_{3} W}^{2} C_{24}\left(H_{3}^{ \pm}, \varphi, H_{3}^{ \pm}\right)+2 C_{24}\left(H_{3}, H_{3}, H_{3}\right)+3 s_{\beta}^{2} C_{24}\left(H_{3}, H_{5}, H_{3}\right)\right]$

$-\lambda_{H_{3} G h}\left[\sum_{\varphi} c_{\varphi W W} c_{\varphi H_{3} W} \tilde{C}_{24}\left(G^{ \pm}, \varphi, H_{3}^{ \pm}\right)+s_{2 \beta} \tilde{C}_{24}\left(G^{ \pm}, H_{5}^{ \pm \pm}, H_{3}^{ \pm}\right)+\frac{s_{2 \beta}}{2} \tilde{C}_{24}\left(G^{0}, H_{5}^{ \pm}, H_{3}^{0}\right)\right]$

$+2 \lambda_{H_{5} H_{5} h}\left[10 C_{24}\left(H_{5}, H_{5}, H_{5}\right)+\frac{10 s_{\beta}^{2}}{3} C_{24}\left(H_{5}, H_{3}, H_{5}\right)\right.$

$\left.+c_{\beta}^{2} C_{24}\left(H_{5}, G^{0}, H_{5}\right)+\frac{7 c_{\beta}^{2}}{3} C_{24}\left(H_{5}, G^{ \pm}, H_{5}\right)\right]$

$+6 \lambda_{h h h}\left[c_{h W W}^{2} C_{24}\left(h, G^{ \pm}, h\right)+c_{h H_{3} W}^{2} C_{24}\left(h, H_{3}^{ \pm}, h\right)\right]$

$+2 \lambda_{H_{1} H_{1} h}\left[c_{H_{1} W W}^{2} C_{24}\left(H_{1}, G^{ \pm}, H_{1}\right)+c_{H_{1} H_{3} W}^{2} C_{24}\left(H_{1}, H_{3}^{ \pm}, H_{1}\right)\right]$

$\left.+2 \lambda_{H_{1} h h}\left[c_{h W W} c_{H_{1} W W} \tilde{C}_{24}\left(h, G^{ \pm}, H_{1}\right)+c_{h H_{3} W} c_{H_{1} H_{3} W} \tilde{C}_{24}\left(h, H_{3}^{ \pm}, H_{1}\right)\right]\right\}$

$+\frac{g^{2}}{64 \pi^{2}}\left\{\frac{80}{3} \lambda_{H_{5} H_{5} h} B_{0}\left(q^{2} ; m_{H_{5}}, m_{H_{5}}\right)+\left(6+10 s_{\beta}^{2}\right) \lambda_{H_{3} H_{3} h} B_{0}\left(q^{2} ; m_{H_{3}}, m_{H_{3}}\right)\right.$

$+6 \lambda_{h h h} c_{h h W W} B_{0}\left(q^{2} ; m_{h}, m_{h}\right)+2 \lambda_{H_{1} H_{1} h} c_{H_{1} H_{1} W W} B_{0}\left(q^{2} ; m_{H_{1}}, m_{H_{1}}\right)$

$+2 \lambda_{H_{1} h h} c_{H_{1} h W W} B_{0}\left(q^{2} ; m_{H_{1}}, m_{h}\right)$

$+2 \lambda_{G G h}\left[2\left(2+c_{2 \beta}\right) B_{0}\left(q^{2} ; m_{G^{ \pm}}, m_{G^{ \pm}}\right)+\left(1+c_{\beta}^{2}\right) B_{0}\left(q^{2} ; m_{G^{0}}, m_{G^{0}}\right)\right]$

$\left.-\lambda_{H_{3} G h s_{2 \beta}}\left[4 B_{0}\left(q^{2} ; m_{H_{3}}, m_{G^{ \pm}}\right)+B_{0}\left(q^{2} ; m_{H_{3}}, m_{G^{0}}\right)\right]\right\}$, 


$$
\begin{aligned}
& \Gamma_{h Z Z}^{1,1 \mathrm{PI}}\left(p_{1}^{2}, p_{2}^{2}, q^{2}\right)=-\sum_{f=t, b, \tau} \frac{4 m_{f}^{2} g_{Z}^{2} N_{c}^{f}}{16 \pi^{2} v}\left\{\left(v_{f}^{2}-a_{f}^{2}\right)\left[B_{0}\left(p_{1}^{2} ; m_{f}, m_{f}\right)+B_{0}\left(p_{2}^{2} ; m_{f}, m_{f}\right)+\left(4 m_{f}^{2}-q^{2}\right) C_{0}(f, f, f)\right]\right. \\
& -\left(v_{f}^{2}+a_{f}^{2}\right)\left[B_{0}\left(p_{1}^{2} ; m_{f}, m_{f}\right)+B_{0}\left(p_{2}^{2} ; m_{f}, m_{f}\right)+2 B_{0}\left(q^{2} ; m_{f}, m_{f}\right)\right. \\
& \left.\left.+\left(4 m_{f}^{2}-p_{1}^{2}-p_{2}^{2}\right) C_{0}(f, f, f)-8 C_{24}(f, f, f)\right]\right\} \\
& +\frac{g^{3} m_{W}}{16 \pi^{2}}\left\{2 c_{W}^{2} c_{h V V} C_{V V V}^{h V V}(W, W, W)+s_{W}^{2} c_{h V V}\left[C_{h V V}^{S V V}\left(G^{ \pm}, W, W\right)+C_{h V V}^{V V S}\left(W, W, G^{ \pm}\right)\right]\right. \\
& -m_{W}^{2} c_{h V V}\left[2 s_{W}^{2} t_{W}^{2} C_{0}\left(W, G^{ \pm}, W\right)+2 \frac{c_{\beta}^{2}}{c_{W}^{2}} C_{0}\left(W, H_{5}^{ \pm}, W\right)+\frac{1}{c_{W}^{6}} \sum_{\varphi} c_{\varphi \mathrm{ZZ}}^{2} C_{0}(Z, \varphi, Z)\right] \\
& -c_{2 W} t_{W}^{2} c_{h V V} \tilde{C}_{24}\left(W, G^{ \pm}, G^{ \pm}\right)+\frac{c_{\beta}^{2}}{c_{W}^{2}} c_{h V V} \tilde{C}_{24}\left(W, H_{5}^{ \pm}, G^{ \pm}\right)+\frac{s_{\beta} c_{\beta}}{c_{W}^{2}} c_{h H_{3} V} \tilde{C}_{24}\left(W, H_{5}^{ \pm}, H_{3}^{ \pm}\right) \\
& +\frac{c_{h Z Z}}{2 c_{W}^{4}} \sum_{\varphi} c_{\varphi Z Z}^{2} \tilde{C}_{24}\left(Z, \varphi, G^{0}\right)+\frac{c_{h H_{3} Z}}{2 c_{W}^{4}} \sum_{\varphi} c_{\varphi H_{3} Z} c_{\varphi Z Z} \tilde{C}_{24}\left(Z, \varphi, H_{3}^{0}\right) \\
& -6 c_{W}^{2} c_{h V V}\left[B_{0}\left(q^{2}, m_{W}, m_{W}\right)-\frac{2}{3}\right] \\
& \left.-s_{W}^{2} t_{W}^{2} c_{h V V} \tilde{B}_{0}\left(W, G^{ \pm}\right)+\frac{2 \sqrt{6}}{3 c_{W}^{2}} c_{\beta} s_{\alpha} \tilde{B}_{0}\left(W, H_{5}^{ \pm}\right)-\frac{1}{4 c_{W}^{4}} \sum_{\varphi}\left(1+\delta_{h \varphi}\right) c_{\varphi V V} c_{h \varphi V V} \tilde{B}_{0}(\varphi, Z)\right\} \\
& +\frac{g_{Z}^{2} m_{Z}^{2}}{16 \pi^{2}}\left\{6 \lambda_{h h h} c_{h V V}^{2} C_{0}(h, Z, h)+2 \lambda_{H_{1} H_{1} h} c_{H_{1} V V}^{2} C_{0}\left(H_{1}, Z, H_{1}\right)\right. \\
& +2 \lambda_{H_{1} h h} c_{h V V} c_{H_{1} V V} \tilde{C}_{0}\left(h, Z, H_{1}\right) \\
& +2 \lambda_{H_{5} H_{5} h}\left[c_{H_{5} Z Z}^{2} C_{0}\left(H_{5}^{0}, Z, H_{5}^{0}\right)+2 c_{W}^{2} c_{\beta}^{2} C_{0}\left(H_{5}^{ \pm}, W, H_{5}^{ \pm}\right)\right] \\
& \left.+4 \lambda_{G G h} s_{W}^{4} c_{W}^{2} C_{0}\left(G^{ \pm}, W, G^{ \pm}\right)\right\} \\
& -\frac{g_{Z}^{2}}{16 \pi^{2}}\left\{\sum _ { \varphi } \left[2 \lambda_{G G h} c_{\varphi Z Z}^{2} C_{24}\left(G^{0}, \varphi, G^{0}\right)+2 \lambda_{H_{3} H_{3} h} c_{\varphi H_{3} Z}^{2} C_{24}\left(H_{3}^{0}, \varphi, H_{3}^{0}\right)\right.\right. \\
& \left.-\lambda_{H_{3} G h} c_{\varphi Z Z} c_{\varphi H_{3} Z} \tilde{C}_{24}\left(G^{0}, \varphi, H_{3}^{0}\right)\right] \\
& +6 \lambda_{h h h} c_{h V V}^{2} C_{24}\left(h, G^{0}, h\right)+2 \lambda_{H_{1} H_{1} h} c_{H_{1} V V}^{2} C_{24}\left(H_{1}, G^{0}, H_{1}\right) \\
& +2 \lambda_{H_{1} h h} c_{h V V} c_{H_{1} V V} \tilde{C}_{24}\left(H_{1}, G^{0}, h\right) \\
& +6 \lambda_{h h h} c_{h H_{3} V}^{2} C_{24}\left(h, H_{3}^{0}, h\right)+2 \lambda_{H_{1} H_{1} h} c_{H_{1} H_{3} V}^{2} C_{24}\left(H_{1}, H_{3}^{0}, H_{1}\right) \\
& +2 \lambda_{H_{1} h h} c_{h H_{3} V} c_{H_{1} H_{3} V} \tilde{C}_{24}\left(H_{1}, H_{3}^{0}, h\right) \\
& +2 \lambda_{H_{5} H_{5} h}\left[\frac{10}{3} C_{24}\left(H_{5}, H_{3}, H_{5}\right)+10 c_{2 W}^{2} C_{24}\left(H_{5}, H_{5}, H_{5}\right)\right. \\
& \left.+c_{H_{5} Z Z}^{2} C_{24}\left(H_{5}, G^{0}, H_{5}\right)+2 c_{\beta}^{2} C_{24}\left(H_{5}, G^{ \pm}, H_{5}\right)\right] \\
& +4 \lambda_{H_{3} H_{3} h}\left[s_{\beta}^{2} C_{24}\left(H_{3}, H_{5}, H_{3}\right)+c_{2 W}^{2} C_{24}\left(H_{3}, H_{3}, H_{3}\right)\right]-\lambda_{H_{3} G h s_{2 \beta}} \tilde{C}_{24}\left(G^{ \pm}, H_{5}^{ \pm}, H_{3}^{ \pm}\right) \\
& \left.+4 \lambda_{G G h}\left[c_{2 W}^{2} C_{24}\left(G^{ \pm}, G^{ \pm}, G^{ \pm}\right)+c_{\beta}^{2} C_{24}\left(G^{ \pm}, H_{5}^{ \pm}, G^{ \pm}\right)\right]\right\} \\
& +\frac{g_{Z}^{2}}{16 \pi^{2}}\left\{5 \lambda_{H_{5} H_{5} h}\left(\frac{1}{3}+c_{2 W}^{2}\right) B_{0}\left(q^{2} ; m_{H_{5}}, m_{H_{5}}\right)\right. \\
& +\frac{\lambda_{H_{3} H_{3} h}}{2}\left(5 s_{\beta}^{2}+2 c_{2 W}^{2}+1\right) B_{0}\left(q^{2} ; m_{H_{3}}, m_{H_{3}}\right)
\end{aligned}
$$




$$
\begin{aligned}
& +\lambda_{G G h}\left[\left(c_{2 W}^{2}+c_{\beta}^{2}\right) B_{0}\left(q^{2}, G^{ \pm}, G^{ \pm}\right)+\frac{1+3 c_{\beta}^{2}}{2} B_{0}\left(q^{2}, G^{0}, G^{0}\right)\right] \\
& -\frac{s_{2 \beta}}{4} \lambda_{H_{3} G h}\left[3 B_{0}\left(q^{2} ; G^{0}, H_{3}^{0}\right)+2 B_{0}\left(q^{2} ; G^{ \pm}, H_{3}^{ \pm}\right)\right] \\
& +\frac{3}{2} \lambda_{h h h} c_{h h V V} B_{0}\left(q^{2}, m_{h}, m_{h}\right)+\frac{1}{2} \lambda_{H_{1} H_{1} h} c_{H_{1} H_{1} V V} B_{0}\left(q^{2}, m_{H_{1}}, m_{H_{1}}\right) \\
& \left.+\frac{1}{2} \lambda_{H_{1} h h} c_{H_{1} h V V} B_{0}\left(q^{2}, m_{H_{1}}, m_{h}\right)\right\} .
\end{aligned}
$$

In the above expressions, we have introduced

$$
\begin{aligned}
\tilde{B}_{0}(A, B) & \equiv B_{0}\left(p_{1}^{2} ; m_{A}, m_{B}\right)+B_{0}\left(p_{2}^{2} ; m_{A}, m_{B}\right), \\
\tilde{C}_{24}(A, B, C) & \equiv C_{24}(A, B, C)+C_{24}(C, B, A), \\
\tilde{C}_{0}(A, B, C) & \equiv C_{0}(A, B, C)+C_{0}(C, B, A),
\end{aligned}
$$

and

$$
\begin{aligned}
& C_{h V V}^{V V V}(X, Y, Z) \equiv\left[17 C_{24}+p_{1}^{2}\left(2 C_{21}+3 C_{11}+C_{0}\right)+p_{2}^{2}\left(2 C_{22}+C_{12}\right)+p_{1} \cdot p_{2}\left(4 C_{23}+3 C_{12}+C_{11}-4 C_{0}\right)\right](X, Y, Z)-3, \\
& C_{h V V}^{S V V}(X, Y, Z) \equiv\left[3 C_{24}+p_{1}^{2}\left(C_{21}-C_{0}\right)+p_{2}^{2}\left(C_{22}-2 C_{12}+C_{0}\right)+2 p_{1} \cdot p_{2}\left(C_{23}-C_{11}\right)\right](X, Y, Z)-\frac{1}{2}, \\
& C_{h V V}^{V V S}(X, Y, Z) \equiv\left[3 C_{24}+p_{1}^{2}\left(C_{21}+4 C_{11}+4 C_{0}\right)+p_{2}^{2}\left(C_{22}+2 C_{12}\right)+2 p_{1} \cdot p_{2}\left(C_{23}+2 C_{12}+C_{11}+2 C_{0}\right)\right](X, Y, Z)-\frac{1}{2} .
\end{aligned}
$$

The 1PI diagram contribution to the $h f f$ vertex is calculated as

$$
\begin{aligned}
& \Gamma_{h f f}^{S, 1 \mathrm{PI}}\left(p_{1}^{2}, p_{2}^{2}, q^{2}\right)=\frac{m_{f}}{16 \pi^{2} v}\left\{-2 g_{Z}^{4} v^{2}\left(v_{f}^{2}-a_{f}^{2}\right) c_{h V V} C_{0}(Z, f, Z)\right. \\
& -4 c_{h f f}\left[e^{2} Q_{f}^{2} C_{h f f}^{F V F}(f, \gamma, f)+g_{Z}^{2}\left(v_{f}^{2}-a_{f}^{2}\right) C_{h f f}^{F V F}(f, Z, f)\right] \\
& +c_{h f f} \frac{m_{f}^{2}}{v^{2}}\left[c_{h f f}^{2} C_{h f f}^{F S F}(f, h, f)+c_{H_{1} f f}^{2} C_{h f f}^{F S F}\left(f, H_{1}, f\right)\right. \\
& \left.-C_{h f f}^{F S F}\left(f, G^{0}, f\right)-\cot ^{2} \beta C_{h f f}^{F S F}\left(f, H_{3}^{0}, f\right)\right] \\
& -c_{h f f} \frac{2 m_{f^{\prime}}^{2}}{v^{2}}\left[C_{h f f}^{F S F}\left(f^{\prime}, G^{ \pm}, f^{\prime}\right)+\cot ^{2} \beta C_{h f f}^{F S F}\left(f^{\prime}, H_{3}^{ \pm}, f^{\prime}\right)\right] \\
& -\frac{m_{f}^{2}}{v}\left\{6 c_{h f f}^{2} \lambda_{h h h} C_{0}(h, f, h)+2 c_{H_{1} f f}^{2} \lambda_{H H h} C_{0}\left(H_{1}, f, H_{1}\right)+2 c_{h f f} c_{H_{1} f f} \lambda_{H_{1} h h} \tilde{C}_{0}\left(h, f, H_{1}\right)\right. \\
& \left.-2 \lambda_{G G h} C_{0}\left(G^{0}, f, G^{0}\right)-2 \cot ^{2} \beta \lambda_{H_{3} H_{3} h} C_{0}\left(H_{3}^{0}, f, H_{3}^{0}\right)-\cot \beta \lambda_{H_{3} G h} \tilde{C}_{0}\left(H_{3}^{0}, f, G^{0}\right)\right\} \\
& +\frac{2 m_{f^{\prime}}^{2}}{v}\left\{2 \lambda_{G G h} C_{0}\left(G^{ \pm}, f^{\prime}, G^{ \pm}\right)+2 \cot ^{2} \beta \lambda_{H_{3} H_{3} h} C_{0}\left(H_{3}^{ \pm}, f^{\prime}, H_{3}^{ \pm}\right)\right. \\
& \left.+\lambda_{H_{3} G h} \cot \beta\left[C_{0}\left(G^{ \pm}, f^{\prime}, H_{3}^{ \pm}\right)+C_{0}\left(H_{3}^{ \pm}, f^{\prime}, G^{ \pm}\right)\right]\right\} \\
& -\frac{g^{2}}{4} c_{h V V}\left[C_{h f f}^{V F S}\left(W, f^{\prime}, G^{ \pm}\right)+C_{h f f}^{S F V}\left(G^{ \pm}, f^{\prime}, W\right)\right] \\
& +\frac{g^{2}}{4} \cot \beta c_{h H_{3} V}\left[C_{h f f}^{V F S}\left(W, f^{\prime}, H_{3}^{ \pm}\right)+C_{h f f}^{S F V}\left(H_{3}^{ \pm}, f^{\prime}, W\right)\right] \\
& -\frac{g_{Z}^{2}}{8} c_{h V V}\left[C_{h f f}^{V F S}\left(Z, f, G^{0}\right)+C_{h f f}^{S F V}\left(G^{0}, f, Z\right)\right] \\
& \left.+\frac{g_{Z}^{2}}{8} \cot \beta c_{h H_{3} V}\left[C_{h f f}^{V F S}\left(Z, f, H_{3}^{0}\right)+C_{h f f}^{S F V}\left(H_{3}^{0}, f, Z\right)\right]\right\},
\end{aligned}
$$


where

$$
\begin{aligned}
C_{h f f}^{F V F}(X, Y, Z) \equiv & {\left[m_{f}^{2} C_{0}+p_{1}^{2}\left(C_{11}+C_{21}\right)+p_{2}^{2}\left(C_{12}+C_{22}\right)\right.} \\
& \left.+p_{1} \cdot p_{2}\left(2 C_{23}-C_{0}\right)+4 C_{24}-1\right](X, Y, Z) \\
C_{h f f}^{F S F}(X, Y, Z) \equiv & {\left[m_{f}^{2} C_{0}+p_{1}^{2}\left(C_{11}+C_{21}\right)+p_{2}^{2}\left(C_{12}+C_{22}\right)\right.} \\
& \left.+2 p_{1} \cdot p_{2}\left(C_{12}+C_{23}\right)+4 C_{24}\right](X, Y, Z)-\frac{1}{2}, \\
C_{h f f}^{V F S}(X, Y, Z) \equiv & {\left[p_{1}^{2}\left(2 C_{0}+3 C_{11}+C_{21}\right)+p_{2}^{2}\left(2 C_{12}+C_{22}\right)\right.} \\
& \left.+2 p_{1} \cdot p_{2}\left(2 C_{0}+2 C_{11}+C_{12}+C_{23}\right)+4 C_{24}\right](X, Y, Z)-\frac{1}{2}, \\
C_{h f f}^{S F V}(X, Y, Z) \equiv & {\left[p_{1}^{2}\left(C_{21}-C_{0}\right)+p_{2}^{2}\left(C_{22}-C_{12}\right)\right.} \\
& \left.+2 p_{1} \cdot p_{2}\left(C_{23}-C_{12}\right)+4 C_{24}\right](X, Y, Z)-\frac{1}{2} .
\end{aligned}
$$

The 1PI diagram contribution to the $h h h$ vertex is given by

$$
\begin{aligned}
& \Gamma_{h h h}^{1 \mathrm{PI}}\left(p_{1}^{2}, p_{2}^{2}, q^{2}\right)=-\sum_{f=t, b, \tau} \frac{8 m_{f}^{4} N_{c}^{f}}{16 \pi^{2} v^{3}}\left[\bar{B}_{0}(f, f)+\left(4 m_{f}^{2}-q^{2}+p_{1} \cdot p_{2}\right) C_{0}(f, f, f)\right] \\
& +\frac{1}{16 \pi^{2}}\left\{g^{3} m_{W}^{3} c_{h V V}^{3}\left[\frac{15}{2} C_{0}(W, W, W)+\frac{15}{4 c_{W}^{6}} C_{0}(Z, Z, Z)\right]\right. \\
& -\frac{g^{3} m_{W}}{2} c_{h V V}^{3}\left[C_{S V V}^{h h h}\left(G^{ \pm}, W, W\right)+\frac{1}{2 c_{W}^{4}} C_{S V V}^{h h h}\left(G^{0}, Z, Z\right)\right] \\
& -\frac{g^{3} m_{W}}{2} c_{h V V} c_{h H_{3} V}^{2}\left[C_{S V V}^{h h h}\left(H_{3}^{ \pm}, W, W\right)+\frac{1}{2 c_{W}^{4}} C_{S V V}^{h h h}\left(H_{3}^{0}, Z, Z\right)\right] \\
& +g^{2} \lambda_{G G h} c_{h V V}^{2}\left[C_{V S S}^{h h h}\left(W, G^{ \pm}, G^{ \pm}\right)+\frac{1}{2 c_{W}^{2}} C_{V S S}^{h h h}\left(Z, G^{0}, G^{0}\right)\right] \\
& +g^{2} \lambda_{H_{3} H_{3} h} c_{h H_{3} V}^{2}\left[C_{V S S}^{h h h}\left(W, H_{3}^{ \pm}, H_{3}^{ \pm}\right)+\frac{1}{2 c_{W}^{2}} C_{V S S}^{h h h}\left(Z, H_{3}^{0}, H_{3}^{0}\right)\right] \\
& -g^{2} \lambda_{H_{3} G h} c_{h V V} c_{h H_{3} V}\left[C_{V S S}^{h h h}\left(W, H_{3}^{ \pm}, G^{ \pm}\right)+\frac{1}{2 c_{W}^{2}} C_{V S S}^{h h h}\left(Z, H_{3}^{0}, G^{0}\right)\right] \\
& -40 \lambda_{H_{5} H_{5} h}^{3} C_{0}\left(H_{5}, H_{5}, H_{5}\right)-24 \lambda_{H_{3} H_{3} h}^{3} C_{0}\left(H_{3}, H_{3}, H_{3}\right) \\
& -8 \lambda_{G G h}^{3}\left[2 C_{0}\left(G^{ \pm}, G^{ \pm}, G^{ \pm}\right)+C_{0}\left(G^{0}, G^{0}, G^{0}\right)\right] \\
& -2 \lambda_{H_{3} H_{3} h} \lambda_{H_{3} G h}^{2}\left[2 \bar{C}_{0}\left(H_{3}^{ \pm}, H_{3}^{ \pm}, G^{ \pm}\right)+\bar{C}_{0}\left(H_{3}^{0}, H_{3}^{0}, G^{0}\right)\right] \\
& -2 \lambda_{G G h} \lambda_{H_{3} G h}^{2}\left[2 \bar{C}_{0}\left(G^{ \pm}, G^{ \pm}, H_{3}^{ \pm}\right)+\bar{C}_{0}\left(G^{0}, G^{0}, H_{3}^{0}\right)\right] \\
& -8 \lambda_{H_{1} H_{1} h}^{3} C_{0}\left(H_{1}, H_{1}, H_{1}\right)-216 \lambda_{h h h}^{3} C_{0}(h, h, h) \\
& -24 \lambda_{H_{1} h h}^{2} \lambda_{h h h} \bar{C}_{0}\left(H_{1}, h, h\right)-8 \lambda_{H_{1} h h}^{2} \lambda_{H_{1} H_{1} h} \bar{C}_{0}\left(H_{1}, H_{1}, h\right) \\
& +c_{h V V} c_{h h V V}\left[2 g^{3} m_{W} \bar{B}_{0}(W, W)+g_{Z}^{3} m_{Z} \bar{B}_{0}(Z, Z)-\left(3 g^{3} m_{W}+\frac{3}{2} g_{Z}^{3} m_{Z}\right)\right] \\
& +4 \lambda_{H_{1} H_{1} h} \lambda_{H_{1} H_{1} h h} \bar{B}_{0}\left(H_{1}, H_{1}\right)+12 \lambda_{H_{1} h h} \lambda_{H_{1} h h h} \bar{B}_{0}\left(H_{1}, h\right)+72 \lambda_{h h h} \lambda_{h h h h} \bar{B}_{0}(h, h) \\
& +10 \lambda_{H_{5} H_{5} h} \lambda_{H_{5} H_{5} h h} \bar{B}_{0}\left(H_{5}, H_{5}\right)+6 \lambda_{H_{3} H_{3} h} \lambda_{H_{3} H_{3} h h} \bar{B}_{0}\left(H_{3}, H_{3}\right) \\
& +2 \lambda_{G G h} \lambda_{G G h h}\left[2 \bar{B}_{0}\left(G^{ \pm}, G^{ \pm}\right)+\bar{B}_{0}\left(G^{0}, G^{0}\right)\right] \\
& \left.+2 \lambda_{H_{3} G h} \lambda_{H_{3} G h h}\left[2 \bar{B}_{0}\left(H_{3}^{ \pm}, G^{ \pm}\right)+\bar{B}_{0}\left(H_{3}^{0}, G^{0}\right)\right]\right\} \text {, }
\end{aligned}
$$


where

$$
\begin{aligned}
& C_{h h h}^{S V V}(A, B, C) \equiv\left[p_{1}^{2}\left(C_{21}-2 C_{11}+C_{0}\right)+p_{2}^{2}\left(C_{22}-C_{12}\right)\right. \\
& \left.+p_{1} \cdot p_{2}\left(2 C_{23}-C_{11}-2 C_{12}+C_{0}\right)+4 C_{24}-\frac{1}{2}\right](A, B, C) \\
& +\left[p_{1}^{2}\left(C_{21}+3 C_{11}+2 C_{0}\right)+p_{2}^{2}\left(C_{22}-C_{12}\right)\right. \\
& \left.+p_{1} \cdot p_{2}\left(2 C_{23}+3 C_{12}-C_{11}-2 C_{0}\right)+4 C_{24}-\frac{1}{2}\right](C, A, B) \\
& +\left[p_{1}^{2}\left(C_{21}+3 C_{11}+2 C_{0}\right)+p_{2}^{2}\left(C_{22}+4 C_{12}+4 C_{0}\right)\right. \\
& +p_{1} \cdot p_{2}\left(2 C_{23}+3 C_{12}+4 C_{11}+6 C_{0}\right)+4 C_{24} \\
& \left.-\frac{1}{2} C_{0}\right](B, C, A) \\
& C_{h h h}^{V S S}(A, B, C) \equiv\left[p_{1}^{2}\left(C_{21}+4 C_{11}+4 C_{0}\right)+p_{2}^{2}\left(C_{22}+2 C_{12}\right)\right. \\
& \left.+p_{1} \cdot p_{2}\left(2 C_{23}+4 C_{12}+2 C_{11}+4 C_{0}\right)+4 C_{24}-\frac{1}{2}\right](A, B, C) \\
& +\left[p_{1}^{2}\left(C_{21}-C_{0}\right)+p_{2}^{2}\left(C_{22}+2 C_{12}\right)\right. \\
& \left.+p_{1} \cdot p_{2}\left(2 C_{23}+2 C_{11}-2 C_{0}\right)+4 C_{24}-\frac{1}{2}\right](C, A, B) \\
& +\left[p_{1}^{2}\left(C_{21}-C_{0}\right)+p_{2}^{2}\left(C_{22}-2 C_{12}+C_{0}\right)\right. \\
& \left.+p_{1} \cdot p_{2}\left(2 C_{23}-2 C_{11}\right)+4 C_{24}-\frac{1}{2}\right](B, C, A), \\
& \bar{C}_{0}(A, B, C) \equiv C_{0}(A, B, C)+C_{0}(C, A, B)+C_{0}(B, C, A), \\
& \bar{B}_{0}(A, B) \equiv B_{0}\left(p_{1}^{2}, m_{A}, m_{B}\right)+B_{0}\left(p_{2}^{2}, m_{A}, m_{B}\right)+B_{0}\left(q^{2}, m_{A}, m_{B}\right) \text {. }
\end{aligned}
$$

[1] G. Aad et al. (ATLAS and CMS Collaborations), J. High Energy Phys. 08 (2016) 045.

[2] S. Dawson et al., arXiv:1310.8361.

[3] K. Fujii et al., arXiv:1506.05992.

[4] S. Kanemura, M. Kikuchi, and K. Yagyu, Nucl. Phys. B907, 286 (2016).

[5] S. P. He and S. h. Zhu, Phys. Lett. B 764, 31 (2017).

[6] S. Kanemura, M. Kikuchi, and K. Yagyu, Nucl. Phys. B917, 154 (2017).

[7] L. Altenkamp, M. Boggia, and S. Dittmaier, J. High Energy Phys. 04 (2018) 062.

[8] S. Kanemura, Y. Okada, E. Senaha, and C.-P. Yuan, Phys. Rev. D 70, 115002 (2004).

[9] A. Arhrib, M. Capdequi Peyranere, W. Hollik, and S. Penaranda, Phys. Lett. B 579, 361 (2004).
[10] S. Kanemura, M. Kikuchi, and K. Yagyu, Phys. Lett. B 731, 27 (2014).

[11] S. Kanemura, M. Kikuchi, and K. Yagyu, Nucl. Phys. B896, 80 (2015).

[12] M. Aoki, S. Kanemura, M. Kikuchi, and K. Yagyu, Phys. Lett. B 714, 279 (2012).

[13] M. Aoki, S. Kanemura, M. Kikuchi, and K. Yagyu, Phys. Rev. D 87, 015012 (2013).

[14] S. Kanemura, M. Kikuchi, K. Sakurai, and K. Yagyu, arXiv:1710.04603.

[15] S. Kanemura, M. Kikuchi, K. Sakurai, and K. Yagyu, Phys. Rev. D 96, 035014 (2017).

[16] H. Georgi and M. Machacek, Nucl. Phys. B262, 463 (1985).

[17] M. S. Chanowitz and M. Golden, Phys. Lett. 165B, 105 (1985). 
[18] W. Konetschny and W. Kummer, Phys. Lett. 70B, 433 (1977).

[19] J. Schechter and J. W. F. Valle, Phys. Rev. D 22, 2227 (1980).

[20] T. P. Cheng and L. F. Li, Phys. Rev. D 22, 2860 (1980).

[21] C. Patrignani et al. (Particle Data Group), Chin. Phys. C 40, 100001 (2016).

[22] C. W. Chiang, A. L. Kuo, and K. Yagyu, J. High Energy Phys. 10 (2013) 072.

[23] C. W. Chiang and K. Tsumura, J. High Energy Phys. 04 (2015) 113.

[24] C. W. Chiang and T. Yamada, Phys. Lett. B 735, 295 (2014).

[25] C. W. Chiang, A. L. Kuo, and K. Yagyu, Phys. Lett. B 774, 119 (2017).

[26] Y. Yamada, Phys. Rev. D 64, 036008 (2001).

[27] J. R. Espinosa and Y. Yamada, Phys. Rev. D 67, 036003 (2003).

[28] S. Blasi, S. De Curtis, and K. Yagyu, Phys. Rev. D 96, 015001 (2017).

[29] T. Blank and W. Hollik, Nucl. Phys. B514, 113 (1998).

[30] S. Kanemura and K. Yagyu, Phys. Rev. D 85, 115009 (2012).

[31] K. Hartling, K. Kumar, and H. E. Logan, Phys. Rev. D 90, 015007 (2014).
[32] C. W. Chiang and K. Yagyu, J. High Energy Phys. 01 (2013) 026.

[33] J. Fleischer and F. Jegerlehner, Phys. Rev. D 23, 2001 (1981).

[34] M. Krause, R. Lorenz, M. Muhlleitner, R. Santos, and H. Ziesche, J. High Energy Phys. 09 (2016) 143.

[35] W. F. L. Hollik, Fortschr. Phys. 38, 165 (1990).

[36] N. K. Nielsen, Nucl. Phys. B101, 173 (1975).

[37] J. M. Cornwall, in Proceedings of the French American Seminar on Theoretical Aspects of Quantum Chromodynamics, Marseille, France, 1981, edited by J. W. Dash (Centre de Physique Theorique, Marseille, 1982); Phys. Rev. D 26, 1453 (1982).

[38] J. M. Cornwall and J. Papavassiliou, Phys. Rev. D 40, 3474 (1989).

[39] G. Passarino and M. J. G. Veltman, Nucl. Phys. B160, 151 (1979).

[40] C. W. Chiang, A. L. Kuo, and T. Yamada, J. High Energy Phys. 01 (2016) 120.

[41] M. Aoki and S. Kanemura, Phys. Rev. D 77, 095009 (2008); 89, 059902(E) (2014).

[42] G. Aad et al. (ATLAS and CMS Collaborations), J. High Energy Phys. 08 (2016) 045.

[43] V. Khachatryan et al. (CMS Collaboration), Phys. Rev. Lett. 114, 051801 (2015). 